
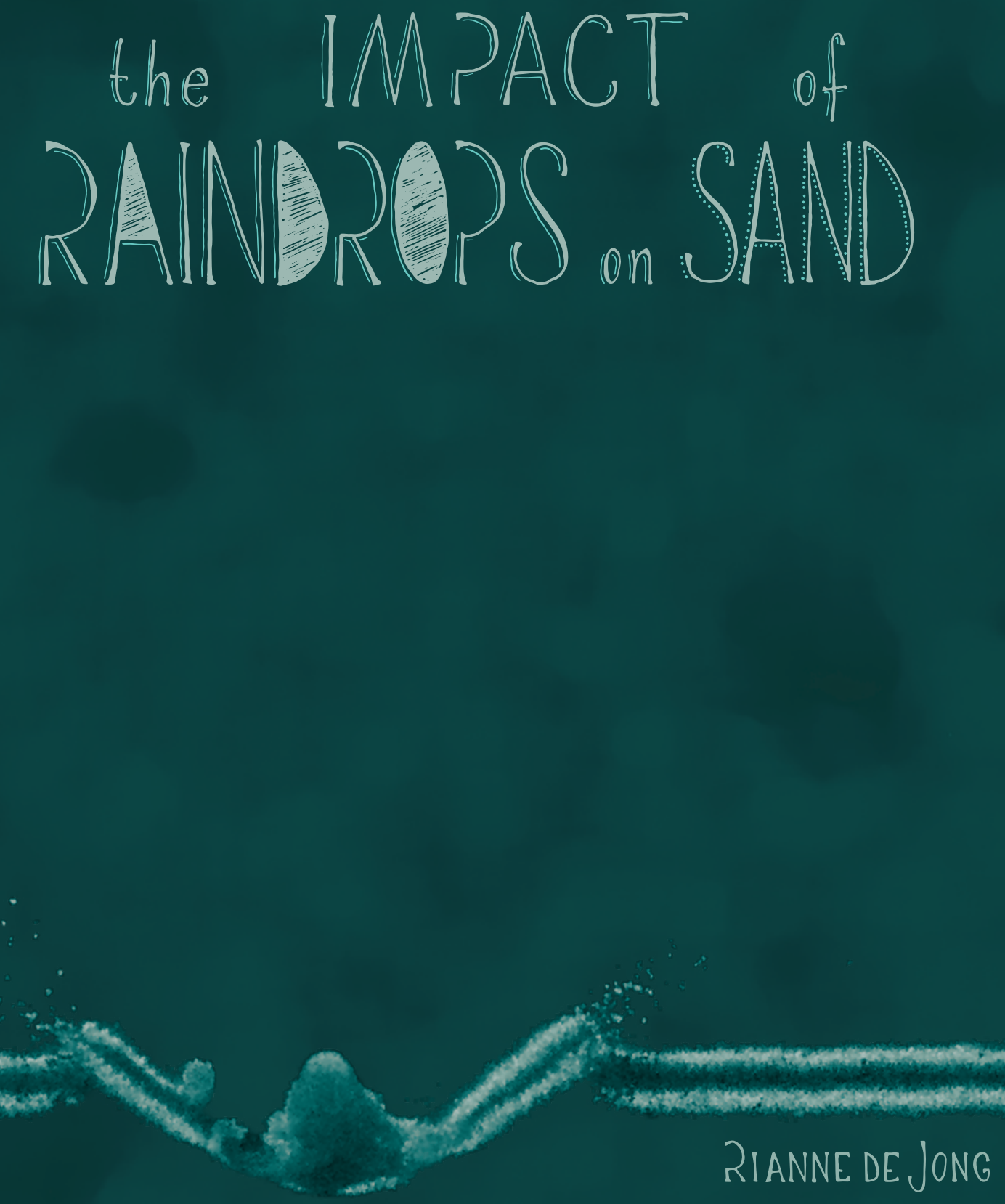



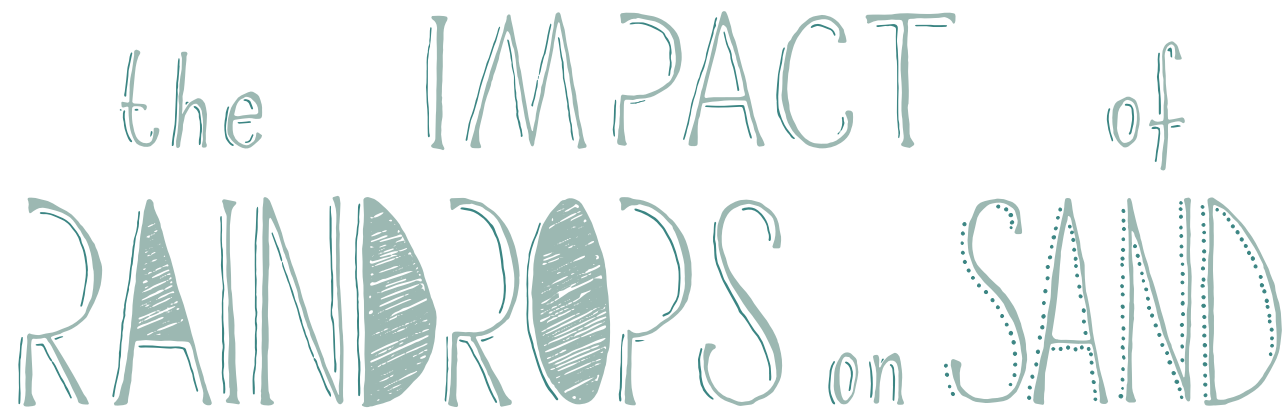

Ri IANNE DE JONG 


\section{Graduation committee:}

Prof. dr. ir. Hans Hilgenkamp (chair/secretary) University of Twente Prof. dr. Devaraj van der Meer (supervisor) University of Twente Prof. dr. ir. Rob G.H. Lammertink University of Twente Prof. dr. ir. Jacco H. Snoeijer University of Twente Prof. dr. Daniel Bonn

Dr. Brian P. Tighe

Dr. Xiang Cheng

University of Amsterdam

Delft University of Technology

University of Minnesota

\footnotetext{
NMO $\begin{aligned} & \text { Netherlands Organisation } \\ & \text { for Scientific Research }\end{aligned}$
}

The work in this thesis was carried out at the Physics of Fluids group of the Faculty of Science and Technology of the University of Twente. This thesis was financially supported by the VIDI program of the Netherlands Organisation for Scientific Research (NWO) under number 68047512.

Dutch title: De impact van regendruppels op zand

Publisher:

Rianne de Jong, Physics of Fluids, University of Twente, P.O. Box 217, 7500 AE Enschede, The Netherlands pof.tnw.utwente.nl, jong.riannede@gmail.com

Cover image: a $3 \mathrm{~mm}$ water droplet impacts with a speed of $4 \mathrm{~m} / \mathrm{s}$ on a sand bed consistent of hydrophobic glass beads of $114 \mu \mathrm{m}$ in size, with a packing density of 0.56 , captured by two laser sheets.

(C) Rianne de Jong, Enschede, The Netherlands 2017

All rights reserved. No part of this publication may be reproduced by any means without the written permission of the publisher.

ISBN: 978-90-365-4357-6

DOI: $10.3990 / 1.9789036543576$ 


\title{
THE IMPACT OF RAINDROPS ON SAND
}

\author{
DISSERTATION
}

to obtain

the degree of doctor at the University of Twente,

on the authority of the rector magnificus,

Prof. dr. T.T.M. Palstra,

on account of the decision of the graduation committee,

to be publicly defended

on Friday the 7th of July 2017 at 12.45 hours

by

Rianne de Jong

born on the 29th of August 1986

in Zwolle, The Netherlands 
This dissertation has been approved by the supervisor:

Prof. dr. Devaraj van der Meer 


\section{Contents}

1 Introduction 1

1.1 Building blocks . . . . . . . . . . . . . . . 2

1.1.1 Impact of a droplet onto a solid substrate. . . . . . . . . . 3

1.1.2 Impact of a solid object onto a granular target . . . . . . . . 5

1.1.3 Liquid penetration into pores between the sand grains . . . . . 9

1.2 In this thesis $\ldots \ldots \ldots \ldots \ldots \ldots \ldots$

2 Adynamic explanation of crater morphologies 13

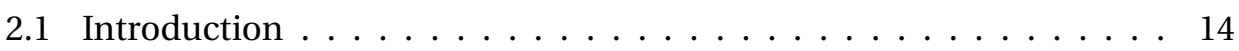

2.2 Experimental methods . . . . . . . . . . . . . . . . . . . . . . . 14

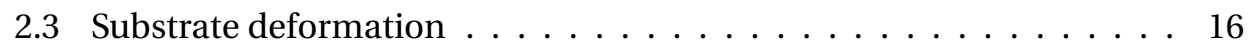

2.3 .1 Deformation speed . . . . . . . . . . . . . . . . . . 17

2.3 .2 Maximum crater depth $\ldots \ldots \ldots \ldots \ldots \ldots$

2.4 Maximum droplet diameter . . . . . . . . . . . . . . 21

2.5 Liquid-grain mixture and the crater morphology . . . . . . . . . 23

2.6 Conclusion . . . . . . . . . . . . . . . . . . . . 26

$3 \quad$ Liquid-grain mixing suppresses droplet spreading and splashing 27

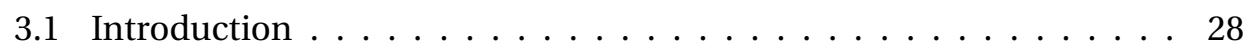

3.2 Experimental methods . . . . . . . . . . . . . . . . . . . 28

3.3 Maximum droplet spreading . . . . . . . . . . . . . . . . . . . . 29

3.4 Effective viscosity . . . . . . . . . . . . . . . . . 31

3.5 Splashing suppression . . . . . . . . . . . . . . . . . . . . . . . . . . . . . . . . . . . . . 34

3.6 Discussion . . . . . . . . . . . . . . . . . . . . . . . . . 35

3. A Effective Weber number $\ldots \ldots \ldots \ldots$. . . . . . . . . . . 37

$3 . \mathrm{B}$ The effective viscosity . . . . . . . . . . . . . . . . . 37

3.C From the capillary to the viscous regime . . . . . . . . . . . 41

3.D Splashing threshold . . . . . . . . . . . . . . . . 42 
4 Crater formation during impact 43

4.1 Introduction . . . . . . . . . . . . . . . . . 44

4.2 Experimental method . . . . . . . . . . . . . . . . . . 45

4.3 Results . . . . . . . . . . . . . . . . . . . . 47

$4.3 .1 \quad$ Final crater profile $\ldots \ldots \ldots \ldots$. . . . . . . . . . . . 47

4.3 .2 Final crater diameter $\ldots \ldots \ldots \ldots$. . . . . . . . . 48

4.3 .3 Crater evolution . . . . . . . . . . . . . . . . . . . . . . 49

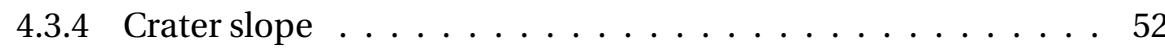

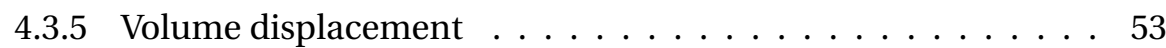

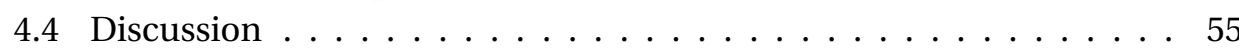

4.4 .1 Maximum crater diameter . . . . . . . . . . . . . 55

4.4 .2 Excavated mass . . . . . . . . . . . . . . . . . . 56

4.4 .3 Crater depth versus diameter . . . . . . . . . . . . 57

4.5 Conclusion . . . . . . . . . . . . . . . . . . . . . . . . . 59

5 Impact cratering in sand: solid intruders vs deformable droplets 61

5.1 Introduction . . . . . . . . . . . . . . . . . . 62

5.2 Experimental methods . . . . . . . . . . . . . . . . . 63

5.3 Maximum crater diameter . . . . . . . . . . . . . . . 64

5.4 Maximum crater depth . . . . . . . . . . . . . . . . . 67

5.5 Excavated volume $\ldots \ldots \ldots \ldots$. . . . . . . . . . . 67

5.5 .1 Packing fraction dependence $\ldots \ldots \ldots$. . . . . . . 68

5.6 Discussion . . . . . . . . . . . . . . . . . . 70

5.A Final crater diameter vs max. drop spreading $\ldots \ldots \ldots \ldots \ldots . . \ldots 72$

5. . Excavated crater volume for small grains . . . . . . . . . . . . . 72

6 Droplet impact near a millimeter-size hole 75

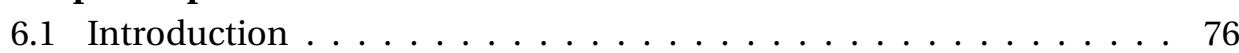

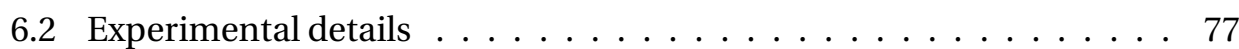

6.3 Impact near pits . . . . . . . . . . . . . . . . . . . . 79

6.3 .1 Observed phenomena . . . . . . . . . . . . . . . . . 79

6.3 .2 Phase diagram . . . . . . . . . . . . . . . 86

6.3 .3 Splash velocity . . . . . . . . . . . . . . . . . 88

6.3 .4 Time until jet formation $\ldots \ldots \ldots \ldots$. . . . . . . 90

6.4 Impact near a pore $\ldots \ldots \ldots \ldots \ldots \ldots \ldots \ldots$

6.4 .1 Observed phenomena . . . . . . . . . . . . . . . . . 91

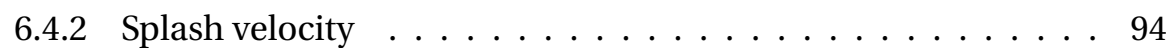

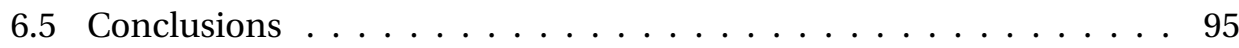


$\begin{array}{lll}7 & \text { Summary and outlook } & 97\end{array}$

$\begin{array}{ll}\text { Bibliography } & 103\end{array}$

$\begin{array}{ll}\text { Samenvatting } & 115\end{array}$

$\begin{array}{ll}\text { Acknowledgments } & 119\end{array}$ 



\section{1 \\ Introduction}

Raindrop impacts on sand or soil leave small indents behind, which are most easily visible at the beach: a crater is formed with some mud located at the center (see figure 1.1). This event is frequently observed in nature in many parts of the world (like the Netherlands), but when absent, they can be artificially produced by sprinklers, generating small drops that hopefully optimally irrigate vegetation. Raindrop impact is not always constructive for agriculture or the natural environment, as it can provoke soil erosion. Already the first impacts of the droplets, on soil that until that moment was still dry, can disintegrate the soil under the force experienced and may seal the surface such that deep liquid penetration gets blocked [1, 2]. Not only in nature the interaction between liquid and granular matter is ubiquitous, but in industry it is present as well, for instance in the process of wet granulation, which is used to produce many pharmaceuticals.

In order to understand and influence this interplay between liquid and sand, the process has been studied recurrently in the accompanying fields (earth sciences, agricultural engineering and powder technology) [3-11]. It is only in the last decade, that drop impact on sand started to draw the attention of physicists, with studies focused on rain showers [12, 13] or single drop impact [14-21].

The work captured within this thesis is embedded in the latter area. We will study the intriguing physics that governs the impact of a single drop on sand. In figure 1.2, we can see that as soon the droplet impacts, it starts to deform, and simultaneously sand is compressed downward and start to splash radially out- 

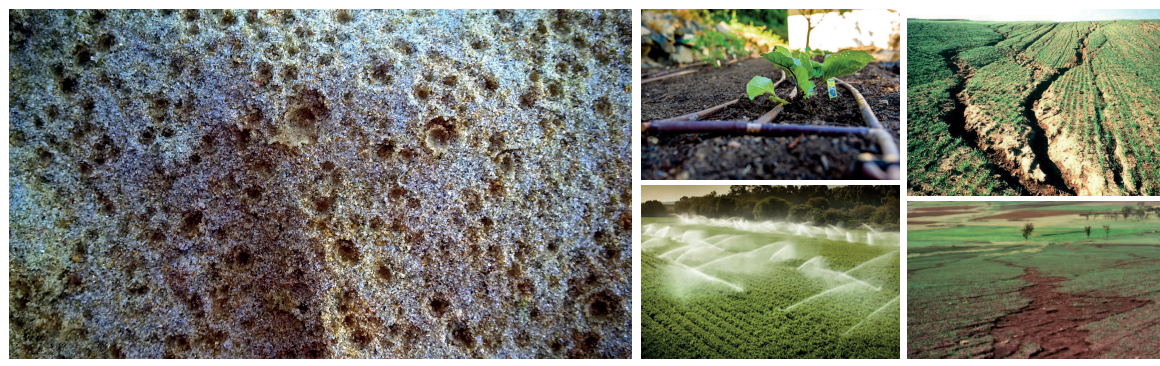

Figure 1.1: Left: raindrop impact on the beach in San Sebastian, Spain. Courtesy: S.C. Zhao. Middle: drip and sprinkler irrigation [22, 23]. Right: rill and sheet erosion [24, 25].

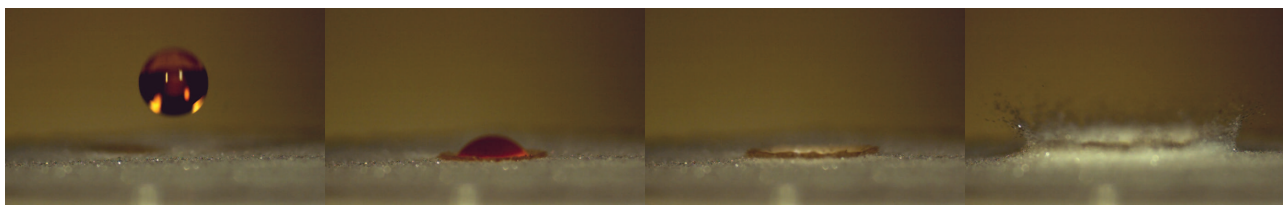

Figure 1.2: A side view of a drop impacting on sand, capturing the initial deformation of the drop and the start of the splash of grains.

wards. Furthermore, the liquid and grains start to mix, resulting in a variety of liquid residues. Observing this phenomenon, various questions can be addressed. What are the mechanisms by which the craters are formed? How is the momentum transferred from liquid to sand? Can we understand what causes the variety of shapes of the liquid-grain mixtures? And what determines the crater shape?

\subsection{Building blocks}

One of the first things to take into account is that the interaction between the liquid drop and the granular bed is subtle and complex: under impact both drop and substrate deform; their deformations are entangled. Similar to the illusion of figure 1.3. where dependent on how you look at it both a young and an elder lady's portrait may be observed, drop deformation and target deformation coexist in our droplet impact problem. However, if we are able to find out what separates them, we may be able to study them simultaneously, similar to the illusion in the figure where we can observe both the young and the elder lady and their traits individually. 


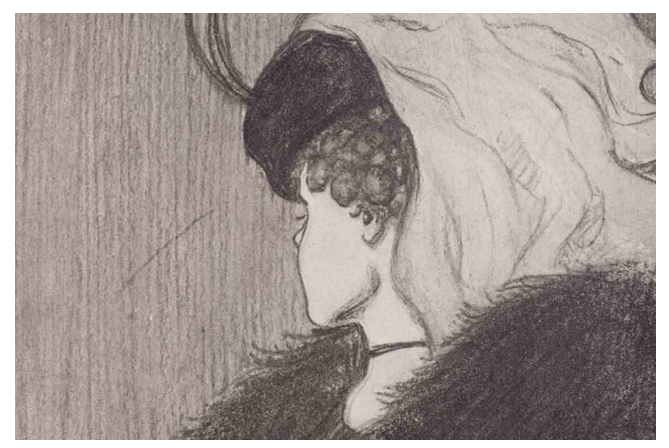

Figure 1.3: The famous illusion of the young versus the elder lady, also known as "my wife and mother-in-law". The earliest version is found on a German postcard, but this one is assigned to W.E. Hill [26]. Taken from [27].

How can we dismantle drop impact on sand? What are the building blocks? The first block we can consider is the deformation of a droplet under forcing (such as impact). Hence, we should look at drop impact on a solid surface, where evidently, the drop is of main interest. Second, we can investigate the intriguing behavior of the granular target upon impact of a solid (i.e., non-deforming) intruder. And lastly, as mixing occurs, we can identify liquid flow into a (nonmoving) porous media. These three topics are concisely discussed below.

\subsubsection{Impact of a droplet onto a solid substrate}

Drop impact on a solid surface has already been studied one-and-a-half century ago [28], and still keeps intriguing scientists [29-31]. The question that is asked most frequently in this area is what controls the droplet spreading, i.e., which forces are important when the droplet spreads. Where part of the literature has focused on estimating the full energy balance between the energy present prior to impact and at maximum droplet spreading, [32-38], others suggest a simple balance of the dominating forces or energies [31, 33, 39]. In this manner, different droplet spreading regimes can be defined. For the aim of this work, we find that the latter path is sufficient in describing the droplet spreading on granular substrates (in chapters 2 and 3 ).

A first energy balance can be acquired by assuming that all of the initial kinetic energy of the impacting drop $E_{k} \propto \rho_{l} D_{0}^{3} U_{0}^{2}$ (with $\rho_{l}$ the liquid density, $D_{0}$ the drop diameter, $U_{0}$ the impact velocity of the droplet) is dissipated by viscosity during spreading. This viscous dissipation scales as $\mu\left(U_{0} / h\right) D_{d}^{* 3}$, where $\mu_{l}$ is the dynamic 
viscosity, and $D_{d}^{*}$ and $h$ are the diameter and the thickness of the drop at maximum spreading respectively. Combining this energy balance with volume conservation $D_{d}^{* 2} h \propto D_{0}^{3}$, this results in $D_{d}^{*} / D_{0} \propto \mathrm{Re}^{1 / 5}$, with the Reynolds number denoted as $\operatorname{Re}=\rho_{l} D_{0} U_{0} / \mu_{l}[29,31,32,36,37,39-42]$.

Another regime can be observed when surface tension, $\sigma$, limits droplet spreading. By assuming that all initial kinetic energy transforms into surface energy $\sim \sigma D_{d}^{* 2}$, this results in $D_{d}^{*} / D_{0} \propto \mathrm{We}^{1 / 2}$ upon maximal spreading, with the Weber number We $=\rho_{l} D_{0} U_{0}^{2} / \sigma$. This scaling is however typically not experimentally observable in droplet spreading on solid substrates, which is attributed to viscous dissipation or break-up of the droplet into a splash [31, 39]. Nonetheless, the maximum spreading diameter of the droplet may be captured by a function that is a combination of these two just mentioned energy balances [36, 42] (referred to as the energy conservation model), which can collapse data with various properties onto a single curve.

An alternative method to account for the surface-tension dominated regime was proposed by Clanet et al. [39]. At the moment the drop impacts, it quickly decelerates as it experiences a stopping force by the substrate. The deceleration of the drop scales as the impact velocity over a typical time scale, i.e., $a=U_{0}^{2} / D_{0}$. The final flattened shape of the drop can accordingly be written as a balance of surface tension by the effective gravity $a$, with the thickness of the flattened, maximally spread out droplet given by $h=\sqrt{\sigma /\left(\rho_{l} a\right)}$, similar to a drop that flattens under the influence of gravity. Combined with volume conservation, it results in a powerlaw with a different scaling exponent $D_{d}^{*} / D_{0} \propto \mathrm{We}^{1 / 4}$. This scaling was found to be consistent with experiments for low-viscous drops of We $>30$ [33, 39, 43-45]. A combination of this scaling with the viscous one, similar to the energy conservation model discussed above, can be used to capture the transition from this capillary regime into the viscous one and has shown to collapse with experimental data [39]. It remains to be understood why some data collapses better with a transition from a $\mathrm{We}^{1 / 2}$ to $\mathrm{Re}^{1 / 5}$ and other experiments with that from $\mathrm{We}^{1 / 4}$ to $\mathrm{Re}^{1 / 5}$.

How does the droplet deform under impact? For a tiny impact velocity, the droplet always retains a shape close to its initial shape and stays a puddle (We $\lesssim 1$ ). For increasing impact velocity, the droplets will become thinnest in the center and a rim is formed at the outer edge, see figure $1.4 a$. When inertia is further increased, the spreading lamella breaks up and splashes, figure $1.4 \mathrm{~b}$. The presence of a surrounding gas is crucial, as it gives rise to an instability at the rim [46-48] and the existence of surface roughness on the target will influence the onset to splashing [30]. In the case that only the droplet properties are varied, a dimensionless splashing parameter can be used, $K_{d} \equiv \mathrm{We}^{1 / 2} \mathrm{Re}^{1 / 4}$. A threshold value for $K_{d}$ can 

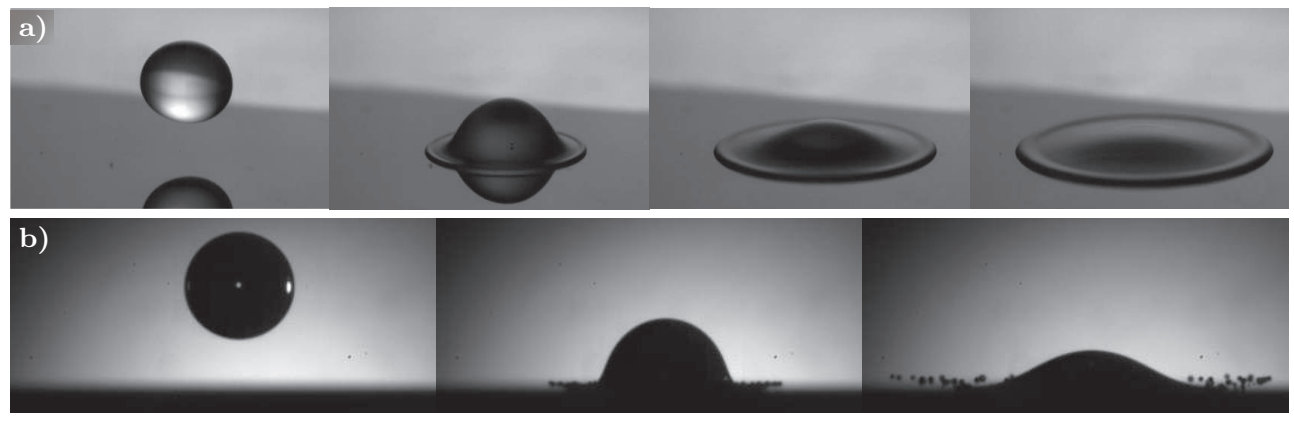

Figure 1.4: Ethanol droplet impact on sapphire. a) For small velocity, the droplet spreads out and a rim is formed. $b$ ) When velocity is increased, the droplet splashes. Credit: M.A.J. van Limbeek

be experimentally obtained that captures the transition from deposition towards splashing [18, 49, 50]. This dimensionless splashing parameter is a phenomenological description of the splash onset, using a balance of inertial forces to viscous and surface tension forces. As mentioned before, the full physical details towards splashing are complex and dependent on the surrounding gas flow and substrate properties [30, 46-48].

\subsubsection{Impact of a solid object onto a granular target}

First, we will introduce some general features of granular materials, since the granular target has intriguing properties that derive from its granular nature. This will then be followed by a brief discussion of the physics of the impact of solid intruders onto sand.

\section{Granular matter}

Sand or soil, sugar or salt, ball bearing balls or ball pit balls, they can all be considered as granular matter. They are macroscopic particles, typically larger than $1 \mu \mathrm{m}$ and, hence, athermal. That is to say, their movements are generally considered not to be influenced by temperature. The fact they are macroscopic gives rise to various problems when describing the behavior they exhibit [56-60]. Here, without pretending to be complete, we will list a few. First, the interaction occurs through the contacts between the grains, which generally results in an inhomogeneous and anisotropic response to external forcing. This can be observed in figure $1.6 a$, where a solid intruder impacts on a (2D) bed of photo-elastic particles. 

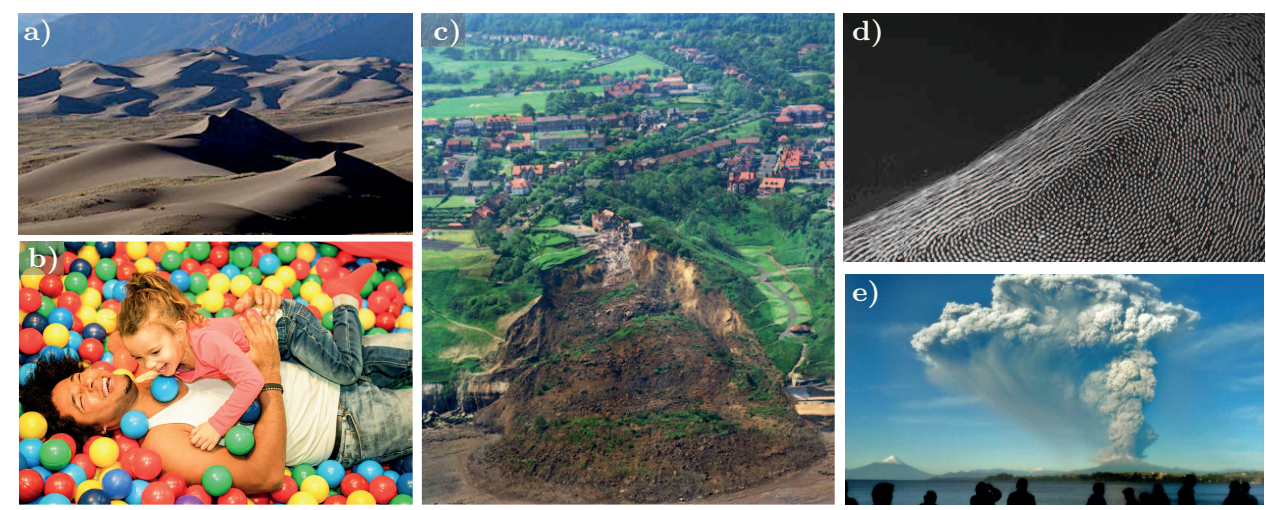

Figure 1.5: Some examples of the various states that granular matter can exhibit: a solid, a liquid and a gas. $a$ ) Desert dunes at rest, where the top layer of grains can be transported under wind flow. $b$ ) Resting on top of ball pit balls. $c$ ) The resultant picture after a landslide occurred. $d$ ) Grains in a rotating drum, partly at rest, and flowing at the top. $e$ ) A volcano outburst. [51-55]

The ones that are compressed, lighten up and we see that the impact force propagates in a chain-like manner (the so-called force chains) through the material [61].

Second, dissipation between the grains is of paramount importance, and is not well captured into a global parameter (such as (plastic) deformability in case of a solid or viscosity for a Newtonian liquid), and there is thus no conservation of energy. Third, there is generally no clear separation of length-scales, i.e., the microscopic (particle) and macroscopic scales (that of hydrodynamic fields, like the density or the velocity) are not clearly separated like in molecular fluids $\mathbf{6 0}$, 63]. All in all, a proper constitutive law to describe granular matter is still work in progress [64, 65].

The common workaround method is to approach the grains either as a solid material, a liquid or a gas [56]. While walking on sand dunes or after a play in a ball pit (figure 1.5 $a, b$ ), one can experience that grains sustain stresses which displays their solid behavior. Flowing grains are seen when pouring cereals into your morning milk or yogurt, as well as in landslides after heavy rain (figure $1.5 c$ ) or in the lab when grains are put in a rotating drum (figure $1.5 \mathrm{~d}$ ). A well-known exposure of a granular gas is displayed in figure 1.5e, where the ash of a volcano is spread out in the air. These three phases can coexist in one configuration, as for instance in the rotating drum picture or an hour glass. The absence of a true phase transition between the phases, makes it a challenge to properly describe granular 


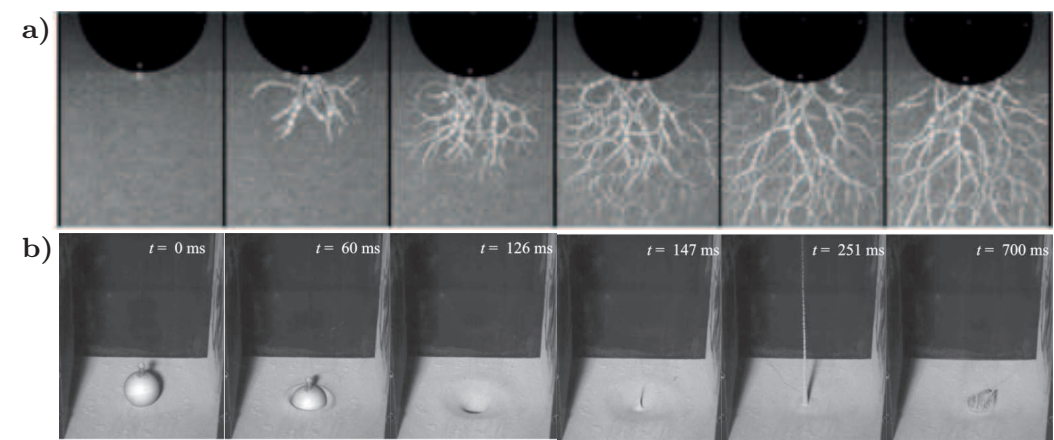

Figure 1.6: a) Impact of solid intruder on a dense 2D bed of photo-elastic particles. Adapted with permission from [61]. Copyrighted by the American Physical Society. b) Impact of an intruder on very loose sand which resembles liquid-like behavior. Also referred to as dry quicksand. Adapted from [62] with permission from Macmillan Publishers Ltd.: Nature, copyright (2004).

matter.

In this study, the sand bed response is between solid-like and fluid-like. An important parameter that affects this behavior is the packing density of the bed, i.e., the volume the grains occupy compared to the total volume of grains and voids. The looser the grains are packed together, the more fluid-like and less solid-like its response will be, of which dry quicksand is an extreme example (figure $1.6 b$ ). Furthermore, under forcing of any kind (e.g., shear or forced intrusion) the volume of the sand bed is modified differently for a loose or a dense bed. For low densities, below a certain critical packing fraction $\phi^{*}$, the bed will compact under forcing. For dense beds, however, the system first has to dilate (i.e., expand) before the grains can flow [66-68]. The effect of packing fraction will be discussed in more detail in chapters 2,4 and 5 .

\section{Impact on sand}

The most severe impacts on granular matter can be found on planets or their satellites, such as our moon (figure 1.7 [69-72]. On the much smaller laboratory-scale, various experiments of dropping a solid object onto sand were performed. Part of these studies [71-73] focused on understanding the full trajectory of the intruder into the bed, i.e., on what causes the intruder to decelerate and eventually come to rest. Various drag force models were found [62, 68, 73-78], of which the main ingredients are inertial drag, viscous (or Stokes') drag, hydrostatic pressure and the 
a)

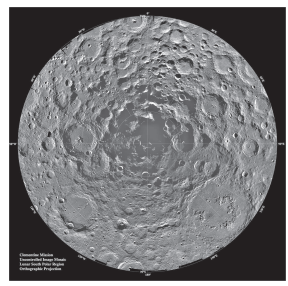

b)

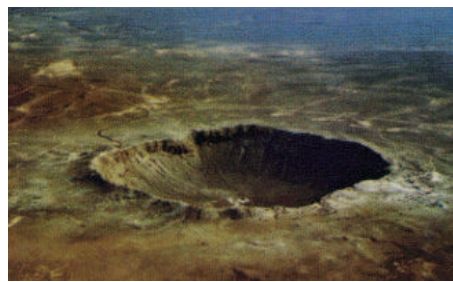

Figure 1.7: $a$ ) Craters on the south pole of the moon. Credit: D. Roddy, NASA. $b$ ) The Barringer crater in Arizona. Credit: NASA/JPLU/USGS.

weight of the intruder. The importance assigned to each of these ingredients in the different models varies greatly. A complementary approach is proposed by Holsapple et al. [69, 79], by considering the relevant dimensionless parameters that set the problem of the formation of (planetary) craters:

$$
\frac{\rho_{i} V_{c}}{m_{i}}=f\left(\frac{U_{0}^{2}}{g D_{0}}, \frac{\rho_{i} U_{0}^{2}}{Y_{t}}, \frac{\rho_{i}}{\rho_{t}}\right)
$$

with on the left side the created crater volume $V_{c}$, intruder mass $m_{i}$ and density $\rho_{i}$ respectively, written as a function $f$ of the Froude number $U_{0}^{2} /\left(g D_{0}\right)$, the dimensionless inverse target strength (or inverse Newton number), and the density ratio of impactor and target. Here, $g$ is the gravitational acceleration, $Y_{t}$ the target yield strength, and $\rho_{t}$ the bulk density of the target. For a granular target, where the bulk density $\rho_{t}$ can be written as $\rho_{g} \phi_{0}$, other non-dimensional parameters can be added into the function $f$, such as the packing fraction $\phi_{0}$, the friction between particles $\eta_{g}$ or the ratio between intruder and grain diameter $D_{0} / d_{g}$.

When only examining variations in the intruder properties, commonly a balance of two energies is proposed, based on two different underlying mechanisms 179, 80]: the impact energy goes either into plastic deformation of the target, proportional to $Y_{t} V_{c}$, or into the lifting of material that is moved out of the crater (i.e., gravitational excavation), proportional to $\rho_{t} g V_{c} Z_{c}$, with $Z_{c}$ the depth of the crater. For the first, if one assumes that the crater volume can be related to one relevant length scale, like the diameter $D_{c}$, such that $V_{c} \propto D_{c}^{3}$, this length scale is proportional to the impact energy to the power $1 / 3$ :

$$
\frac{D_{c}}{D_{0}} \propto\left(\frac{\rho_{i} U_{0}^{2}}{Y}\right)^{1 / 3}
$$


For the latter, we obtain a power-law exponent 1/4:

$$
\frac{D_{c}}{D_{0}} \propto\left(\frac{\rho_{i}}{\rho_{t}} \frac{U_{0}^{2}}{g D_{0}}\right)^{1 / 4}
$$

Interestingly, many [80-82] find for the crater diameter a power-law exponent of $1 / 4$ with energy, suggesting gravitational excavation as main energy sink, but for the crater depth a power-law exponent of $1 / 3$ is obtained [74, 82], which indicates plastic deformation. Also more complicated relations between diameter and depth are reported [71, 83]. In the field of impact on sand, variations in the packing fraction are hardly studied systematically [68]. We will discuss these scalings further in chapter 4 , where we look into the crater formation after a droplet impacts.

\subsubsection{Liquid penetration into pores between the sand grains}

In 1856 a book was published by Henri Darcy discussing the water distribution in the town Dijon. Tucked away in an appendix, an empirical derivation on the flux, $\vec{q}$ of water through a porous medium (sand) was derived, which is now known as Darcy's law: $\vec{q}=-\frac{\kappa}{\mu_{l}} \vec{\nabla} P$, with $\kappa$ the permeability of the material and $\vec{\nabla} P$ the pressure gradient that will drive the liquid into the pores [84]. This relation indicates that the larger the pressure exerted on the liquid, or the easier it is for liquid to flow into the pores (higher permeability), the higher the flux into the the porous medium. For drop impact on a porous substrate, the driving pressure can be either caused by the inertia of the impacting droplet, $P_{i}=\rho_{l} U_{0}^{2}$, or result from capillary suction, $P_{c}=2 \sigma \cos \theta / r$, with $\theta$ the (effective) contact angle of the liquid on the pore surface and $r$ the pore radius. Depending on the wettability of the substrate, the capillary pressure will either prevent liquid to enter the pores (hydrophobic, $\theta>90^{\circ}$ ) or promote penetration (hydrophilic, $\theta<90^{\circ}$ ). We will relate the penetration of liquid into pores to the mixing of liquid and grains in chapters 2 and 3 ,

\subsection{In this thesis}

In this thesis, we study single droplet impact on sand to unravel the underlying physics of the crater formation process. We will start with focusing on the droplet deformation and mixing in the first two chapters, subsequently shift towards the deformation of the sand in chapters 4 and 5 , and finally discuss a comparison of drop impact near closed and open-ended holes. 
The main focus of chapter 2 is the explanation of the variety of liquid-grain shapes observed after impact. We find that the transition between curved, compact residues and flat ones can be explained by a transition from a situation where little mixing between liquid and grains occurs and the liquid-grain mixture is still able to contract, to one where mixing is dominant and all kinetic energy of the droplet is lost during its spreading phase. Various ingredients are needed to predict this transition, of which an estimate of the amount of the initial droplet energy that is going either into droplet deformation or into deformation of the sand is of key importance. In fact, we find that this energy partitioning is applicable in all the cases that we have studied in this thesis.

We further deepen our understanding of liquid-grain mixing in chapter 3 where we study how it affects the maximum spreading diameter and splashing threshold of the droplet. We find that the mixing between liquid and grains can be modeled similar to the growth of a viscous boundary layer which develops when a liquid drop spreads on a solid surface. Using this as an input for a modified Reynolds number, we will show that the maximum spreading of the droplet goes from a capillary regime of little mixing, towards a viscous regime where mixing is dominant. Furthermore, we demonstrate that the splashing threshold for the spreading liquid can be captured with the same modified non-dimensional numbers.

In chapter 4, we focus on the target deformation and investigate by which mechanisms the crater is formed. We compare the final crater shape with its shape at the moment during impact at which the crater reaches its maximum depth. We find that the packing density of the bed affects initially only the crater depth and not its diameter, but when the crater develops further the diameter of the crater is altered as well. Looking into various measurable quantities, we provide evidence that avalanches become more pronounced for looser packing fractions and larger impact velocities, leading in these cases to broader craters.

Next, in chapter[5, we investigate the effect of the deformability of the intruder by comparing the impact of a droplet on a granular bed with that of a solid intruder of similar size and density. We find that droplets produces craters that are relatively broad and shallow when compared to those formed by solid intruders. Nonetheless, we show that the excavated crater volume is similar. We trace this back to the deformability of the impacting drop, by which the droplet momentum is not only transferred vertically to the bed, but also partly radially outwards.

A last aspect that we want to elucidate on, is what happens for drop impact on an uneven non-deformable surface, similar to the top layer of a sand bed. In chapter 6, we look into the simplified and well-controlled case of a droplet that im- 


\subsection{IN THIS THESIS}

pacts onto a solid substrate close to a large-scale roughness, namely a millimetersize hole. We find that drop impact near a closed version (pit) or an open version (pore), where air may escape through the bottom even if the top part of the hole is sealed by the spreading droplet, result in different phenomena. For both type of holes, splashing at the hole edge occurs, however, we find that for the closed-end pit the splash is much more pronounced in volume and speed and postulate that the air present inside it plays a key role, as it will redirect the spreading lamella. 



\section{2}

\section{A dynamic explanation of crater morphologies}

$\|$

As a droplet impacts upon a granular substrate, both the intruder and the target undergo deformation, during which the liquid may penetrate into the substrate. These three aspects together distinguish it from other impact phenomena in the literature. We perform high-speed, double-laser profilometry measurements and disentangle the dynamics into three aspects: the deformation of the substrate during the impact, the maximum spreading diameter of the droplet, and the penetration of the liquid into the substrate. By systematically varying the impact speed and the packing fraction of the substrate, (i) the substrate deformation indicates a critical packing fraction $\phi^{*} \approx 0.585$; (ii) the maximum droplet spreading diameter is found to scale with a Weber number corrected by the substrate deformation; and (iii) a model of the liquid penetration is established and is used to explain the observed crater morphology transition.

This chapter is published as: Song-Chuan Zhao, Rianne de Jong, and Devaraj van der Meer, "Raindrop impact on sand: a dynamic explanation of crater morphologies" Soft Matter 11, 65626568 (2015). 


\subsection{Introduction}

Liquid droplets impacting on a granular layer are very common in nature, industry, and agriculture, ranging from raindrops falling onto the desert sand or soil to granulation in the production process of many pharmaceuticals. In spite of the fact that it is common, the physical mechanisms involved in the impact of a droplet on sand did not attract much attention until recently $[6,11,14-21,85,86]$, and the underlying physics is still largely unexplored. In contrast, droplet impact on a solid surface or a liquid pool has been studied extensively [29, 36, 39]. However, a granular substrate can act both solid-like and fluid-like [56] and many experiments have been conducted to reveal the response of a granular packing to a solid object impact [68, 71, 75, 80, 82, 87, 88], where the intruder does not deform. Droplet impact on a granular substrate adds new challenges to the above: First, both the intruder and the target, not merely one of the two, deform during impact; second, the liquid composing the droplet may penetrate into the substrate during the impact and may, in the end, completely merge with the grains. These complex interactions between the droplet intruder and the granular target create various crater morphologies as reported in the literature [14, 15, 17, 21, 86], see figure 2.1 for examples. An appealing and natural question is by what mechanism craters are formed and how this leads to the observed rich morphological variation. This is the main focus of this chapter. Quantitative dynamic details, e.g., on the deformation of droplet and substrate in response to the impact, are necessary to gain insight about this issue. Previous dynamic measurements only regard the droplet spreading [15, 17, 18] or individual splashing grains [20], and a systematical study of the effect of the packing fraction of the substrate is still missing. In this chapter, we perform dynamic measurements with high-speed laser profilometry and study the dependence of the dynamics on impact speed and packing fraction of the granular substrate. Following the discussion about the results of the substrate deformation and the droplet deformation and the underlying physics, a quantitative model is established which explains the observed crater morphologies in the end.

\subsection{Experimental methods}

A water droplet with diameter $D_{0}=2.8 \mathrm{~mm}$ acts as the intruder. The water droplet is pinched off from a needle at rest and dropped from a certain height. The droplet is accelerated by gravity and impacts on the horizontal surface perpendicularly. The impact speed, $U_{0}$, is computed from a calibrated height-speed profile. Rain 


\subsection{EXPERIMENTAL METHODS}

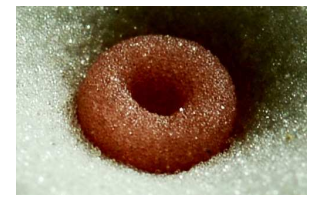

doughnut

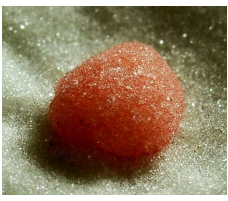

truffle

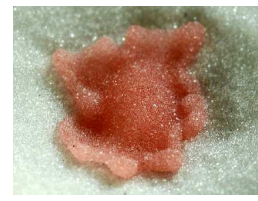

pancake

Figure 2.1: Various crater morphologies observed in the experiments are categorized as: doughnut, truffle, and pancake.

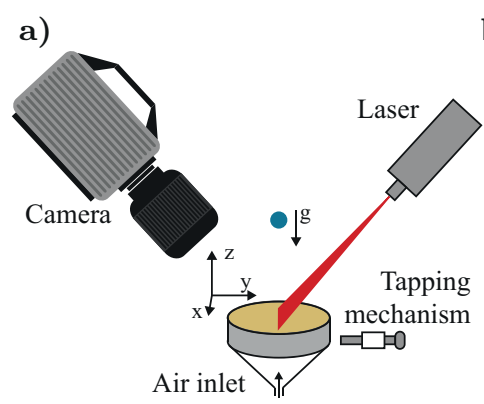

b)

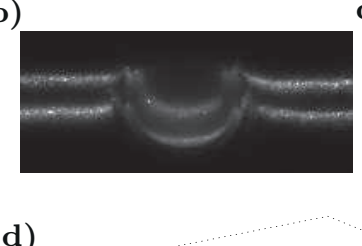

d)

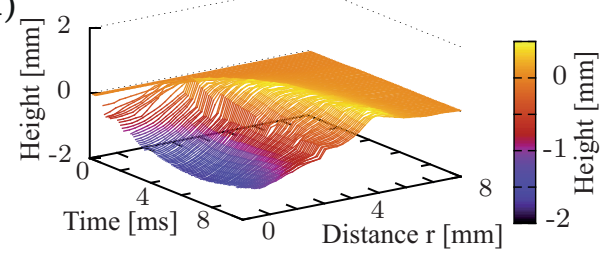

Figure 2.2: $a$ ) Setup sketch. The height, $z(x, y)$, is measured as a function of coordinates on the horizontal $x y$ plane with laser sheets and a high speed camera. $b$ ) An image taken during the impact, where the droplet is at its maximum deformation. Two laser lines are used for high-speed profilometry, where the deflection of the laser lines indicates the deformation of the target surface. $c$ ) The laser line deflection in $b$ ) is used to reconstruct the height, $z$, as a function of the distance to the impact center, $r$. The dashed line indicates the initial surface height, $z=0$ and the center depth is labeled $Z_{c}$. $d$ ) Time evolution $z(r, t)$ of the crater depth. 
consists of droplets with a maximum diameter $6 \mathrm{~mm}$ [89] falling at their terminal velocity. For a droplet diameter of $2.8 \mathrm{~mm}$, the terminal velocity is about $7 \mathrm{~m} / \mathrm{s}$. In this chapter however, the impact speed is one of the important control parameters and is varied from $1.35 \mathrm{~m} / \mathrm{s}$ to $4.13 \mathrm{~m} / \mathrm{s}$. The target granular packing consists of polydisperse soda lime glass beads with diameter $d_{g}=70-110 \mu \mathrm{m}$ and specific density $\rho_{g}=2500 \mathrm{~kg} / \mathrm{m}^{3}$. While there are studies considering the effect of the wettability of grains $[6,19]$, the wettability is not varied in this chapter, where hydrophilic grains are used. Grains are put into an oven at $90^{\circ} \mathrm{C}$ for at least half hour before experiments, after which we let them cool down to room temperature. Various packing fractions, $\phi_{0}$, are prepared via air fluidization and a tapping device (figure 2.2 $a$ ). The initial packing fraction $\phi_{0}$ is computed according to the surface height relative to the container edge before impact. During the impact, the height of the substrate surface, $z(x, y)$, is measured by high-speed laser profilometry with a typical depth resolution of $0.1 \mathrm{~mm}$ per pixel. The impact process is captured by a high speed camera (Photron SA-X2) with a frame rate of $10000 \mathrm{~Hz}$, of which an experimental image is shown in figure 2.2 $b$. For dynamic analysis, the surface height before impact is taken as the reference $z=0$. We use two laser lines for the profilometry, and, as the surface deforms, the lines are deflected. We translate the deflection into the height function, $z(r)$, where $r$ is the distance to the impact center in the horizontal plane, i.e., $r=\sqrt{\left(x-x_{c}\right)^{2}+\left(y-y_{c}\right)^{2}}$, where $x_{c}, y_{c}$ are the coordinates of the impact center. Assuming axisymmetry, the impact center is located by determining when the deflections reconstructed from individuals laser lines match. Henceforth, the height function, $z(r)$, used for further analysis is always averaged over the two laser lines. An example of $z(r)$ and its time evolution are illustrated in figures $2.2 c$ and $d$.

\subsection{Substrate deformation}

We use the depth of the crater center, $Z_{c}(t)$ as defined in figure $2.2 \mathrm{c}$, to characterize the development of the crater. A typical temporal evolution of the crater depth is shown in figure 2.3. In the beginning the crater becomes deeper with time. At a certain moment, the crater reaches its maximum depth $Z_{c}^{*}$, after which the droplet contracts and transports grains mixed with it towards the crater center. An avalanche subsequently occurs at a longer time scale. Both of these effects tend to decrease $Z_{c}$, i.e., make the crater shallower. Here, we focus only on the early stage of $Z_{c}(t)$ and quantify its evolution by measuring two quantities: the 


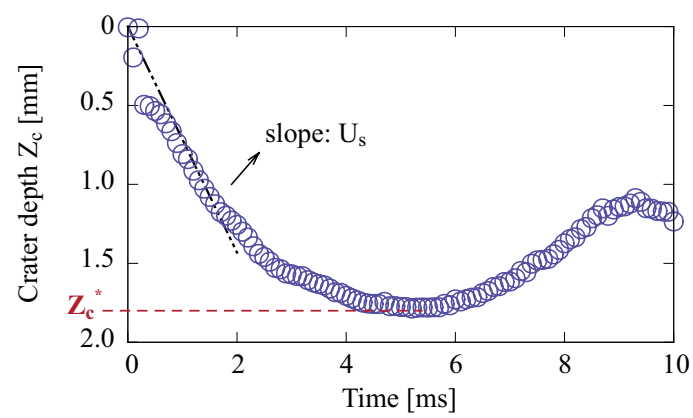

Figure 2.3: Crater depth versus time. The maximum crater depth is denoted as as $Z_{c}^{*}$. A straight line is fitted on the data points of $t \leq\left(D_{0}+2 Z_{c}^{*}\right) / U_{0}$. The slope of this fit, $U_{s}$, is used to indicate the speed of the deformation. Here, $U_{0}=3.19 \mathrm{~m} / \mathrm{s}$, and $\phi_{0}=0.569$.

initial speed of the deformation, $U_{s}$, and the maximum crater depth, $Z_{c}^{*}[$. The behavior of these two quantities is essential to understand not only the response of the granular substrate, but also the deformation of the droplet and the formation of various crater shapes.

\subsubsection{Deformation speed}

The initial deformation speed $U_{s}$ is determined as the slope of a linear fit of $Z_{c}(t)$ within a time duration $t \leq t_{\text {imp }}$, where $t_{\text {imp }}=\left(D_{0}+2 Z_{c}^{*}\right) / U_{0}$ denotes the impact time scale (figure 2.3). The ratio of the impact velocity and this deformation speed is plotted in figure 2.4 $a$ for all experiments. This ratio is largely independent of impact speed which implies that it is an inherent property of the granular substrate. On the other hand, the dependence on the packing fraction $\phi_{0}$ indicates a transition around $\phi^{*} \approx 0.585$. Here, we introduce a simple scenario to elucidate this transition. Upon impact the droplet - with a mass $M_{d}$ - accelerates a certain amount of grains in the vertical direction to the initial deformation speed $U_{s}$, while by vertical momentum conservation the momentum lost by the droplet

\footnotetext{
${ }^{1}$ When the droplet reaches its maximum spreading diameter, its average thickness $h=$ $(2 / 3) D_{0}\left(D_{0} / D_{d}^{*}\right)^{2}$. According to the geometric configuration of the experiments, the depth difference introduced by this thickness is $0.37 \mathrm{~h}$. For the smallest $U_{0}$, the least spreading case, where the refraction introduces the largest error, this estimation gives a height difference of $0.14 \mathrm{~mm}$, which is close to the depth resolution of the laser profilometry system. Moreover, this refraction error is well below the smallest measured $Z_{c}^{*}$ of $0.5 \mathrm{~mm}$. Since the center of the impact droplet is even thinner than $h$ as estimated above, the influence on the maximum center depth measurement would be even weaker in practice.

${ }^{2}$ Although the measurement of $U_{s}$ is merely the first approximation of the deformation speed, we confirm that $\phi^{*}$ is not altered by reducing the fitting region to half its size.
} 
a)

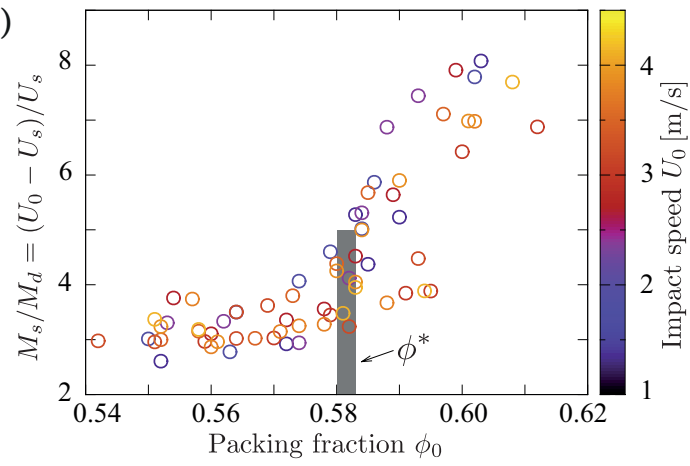

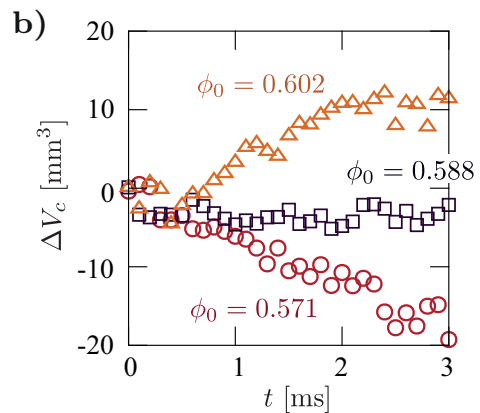

Figure 2.4: $a$ ) The ratio of the impact velocity $U_{0}$ and the deformation speed of the substrate $U_{s}$ as a function of packing fraction $\phi_{0}$. The Y-axis, $\left(U_{0}-U_{s}\right) / U_{s}$, indicates the amount of grains involved in the deformation $M_{s}$ normalized by the droplet mass $M_{d}$ (see text), where $U_{s}$ is the speed of the substrate deformation (figure 2.3. $b$ ) The volume change of the substrate, $\Delta V_{c}$, for experiments with the same impact speed $U_{0}=3.75 \mathrm{~m} / \mathrm{s}$, but three different packing fractions, $\phi_{0}=0.571,0.588,0.602$, from bottom to top.

is proportional to $M_{d}\left(U_{0}-U_{s}\right)$. Thus, the mass of grains accelerated by the droplet is $\left(U_{0}-U_{s}\right) / U_{s}$ times larger than the mass of the droplet. This ratio, plotted in figure $2.4 a$, therefore indicates the amount of substrate material involved in the deformation dynamics and the yield stress of the substrate, i.e., the larger this ratio is, the harder is the substrate. With this interpretation, the observed transition at $\phi^{*}$ in figure $2.4 a$ is reminiscent of the penetration force transition which points to the dilatancy transition [66].

The dilatancy transition expresses a peculiar phenomenon that shearing a granular packing above a threshold packing fraction results in dilation (i.e., expansion along the perpendicular directions), whereas a loose packing is just compactified under such a perturbation [67]. Though the word 'dilation' describes a change of the volume, previous studies have shown that the dilatancy transition is accompanied by a force response transition $[66,68,88,90]$. As explained above, this force transition feature is already encapsulated by the factor $\left(U_{0}-U_{s}\right) / U_{s}$ representing momentum transfer, and the volume change of the substrate is supposed to give a transition at $\phi^{*}$ as well. We compute the volume change as the integral, $\Delta V_{c}=\pi \int z(r) d r^{2}$, for each frame. The result is plotted in the figure 2.4b for three experiments with different $\phi_{0}$ but the same impact speed $U_{0}$. The volume change is negative (i.e., the substrate compactifies) for the loosest substrate and indeed increases to positive (indicating dilation) with increasing $\phi_{0}$. Based on 
the above evidence we define a critical packing fraction $\phi^{*} \approx 0.5853$. The grains underneath the droplet intruder are forced to rearrange to $\phi^{*}$ during the impact whatever the initial packing fraction $\phi_{0}$ is. This value will be used to model the mixing between liquid and grains.

\subsubsection{Maximum crater depth}

In contrast to figure 2.4 the maximum crater depth, $Z_{c}^{*}$ plotted in figure 2.5 $a$, depends on both the initial packing fraction, $\phi_{0}$, and the impact speed of the droplet, $U_{0}$. It has been shown above that a dense substrate is more difficult to deform, therefore it should come as no surprise to see that the maximum crater depth $Z_{c}^{*}$ decreases with $\phi_{0}$. The increase of $Z_{c}^{*}$ with the impact speed $U_{0}$ is also anticipated: a higher impact energy generates a deeper crater.

Studies on solid intruder impact on a granular substrate [75, 80, 82] can help to further understand the impact speed dependence. For a crater created by a solid object, two scaling laws of its depth with impact energy are suggested based on how the kinetic energy, $E_{k}$, is dissipated [80]. Assuming that plastic deformation is the most dissipating process, the crater volume scales with the kinetic energy, which yields $Z_{c}^{*} \sim E_{k}^{1 / 3}[75,82]$. On the other hand, if material ejection absorbs most of the impact energy, then the converted gravitational energy leads to $Z_{c}^{*} \sim$ $E_{k}^{1 / 4}$. The volume change in figure $2.4 b$ implies strong plastic deformations during the impact. However, in the case of droplet impact, not only the substrate but also the intruder dissipates the impact energy via deformations. To apply the above arguments, we therefore first need to decide upon the distribution of $E_{k}$ between the droplet and the granular packing.

The droplet experiences a deceleration force. This force does work on both the droplet and the granular target along the total displacement, $\frac{1}{2} D_{0}+Z_{c}^{*}$. This work transforms the impact energy $E_{k}=M_{d} U_{0}^{2} / 2$ into other energy forms, such as surface energy of the droplet and dissipation inside the substrate. Out of the total displacement, $\frac{1}{2} D_{0}$ is the contribution of the droplet deformation, while $Z_{c}^{*}$ is that of the substrate deformation. Such a force, $E_{k} /\left(\frac{1}{2} D_{0}+Z_{c}^{*}\right)$, indicates the average interaction between droplet and the substrate, from which we can estimate the energy absorbed by the droplet $E_{d}$ and that absorbed by the substrate $E_{s}$ as the work

\footnotetext{
${ }^{3}$ Note that these measurements pertain to the initial deformation of the bed, i.e., before liquidgrain mixing becomes important. The dilation and its transition reported here are inherent properties of granular packings, independent of the intruder properties. This is different to the dilation measured from the final crater volume [16], where the residue of the liquid-grain mixture plays a very important role.
} 
a)

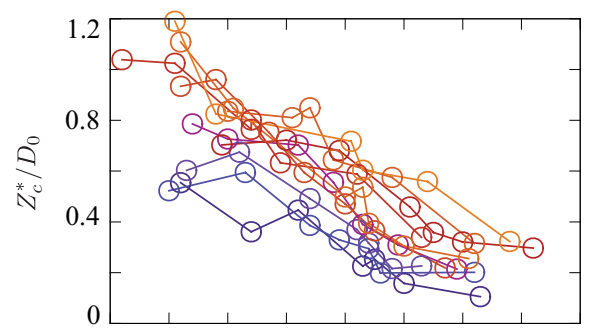

b)
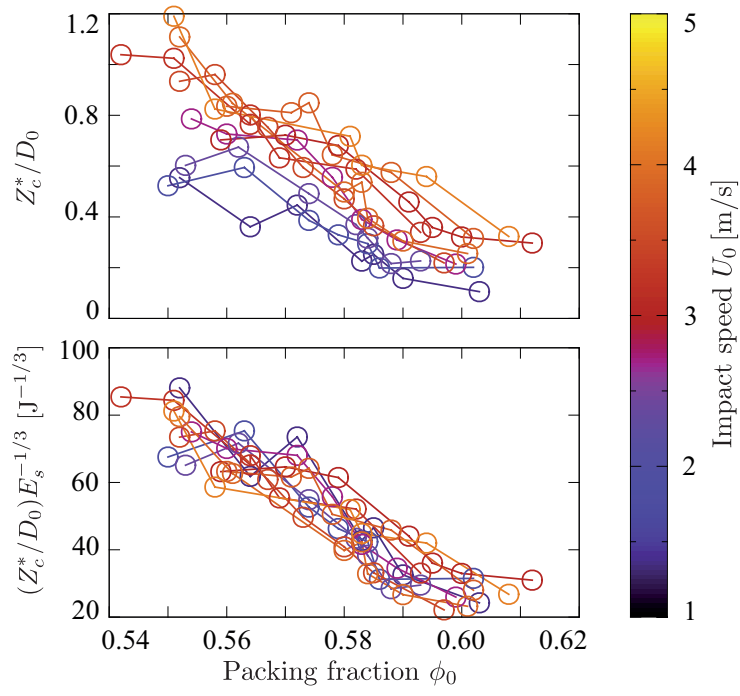

Figure 2.5: a) Maximum crater depth $Z_{c}^{*}$ (non-dimensionalized with the droplet radius $D_{0}$ ) versus packing fraction $\phi_{0}$. The impact velocity, $U_{0}$, is color coded. The dependence on $\phi_{0}$ is stronger than that on $U_{0} . b$ ) Maximum crater depth scaled with the cubic root of the energy transferred into the substrate, $E_{s}$, again versus $\phi_{0}$. See text and equation 2.1 for details.

done by this interaction force to deform the droplet and substrate respectively:

$$
E_{d}=\frac{D_{0}}{D_{0}+2 Z_{c}^{*}} E_{k} ; E_{s}=\frac{Z_{c}^{*}}{D_{0} / 2+Z_{c}^{*}} E_{k}
$$

This energy distribution is estimated using the average force between the intruder and the target. While it has been shown that the impact force between a droplet and a solid surface is time dependent [36, 291], the subsequent analysis justifies equation 2.1 by hindsight.

With measured $Z_{c}^{*}$ and $E_{k}$, the energy distributed into the substrate $E_{s}$ can be computed for all experiments. We find that $Z_{c}^{*} \propto E_{s}^{1 / 3}$ is the better relation to collapse the dependence on $U_{0}$ (figure 2.5b). For a given $\phi_{0}$, a power law fit gives $Z_{c}^{*} \propto E_{s}^{\alpha}$ with $\alpha=0.33 \pm 0.04$. This scaling suggests plastic deformation as the main cause of energy dissipation, which stands to reason. We will show that the kinetic energy distribution in equation 2.1 has essential consequences to the droplet deformation and the crater morphology as well. 


\subsection{Maximum droplet diameter}

In contrast to solid object impact, the droplet deforms during impact [15, 17, 18]. This droplet deformation changes the contact area between liquid and grains, which will turn out to play an important role in determining the crater morphology. In this section we focus on the maximum spreading of the droplet.

For most of our experiments, the droplet contracts after spreading, which implies that surface tension is the dominant stopping force of droplet deformation. In this regime, the Weber number $\mathrm{We}=\rho_{l} D_{0} U_{0}^{2} / \sigma$ is the expected relevant dimensionless parameter measuring the relative importance of the kinetic energy of the impacting droplet and the surface energy. Here, $\rho_{l}$ is the density of the liquid and $\sigma$ is its surface tension. Previous studies about droplet impact on a solid substrate have proposed two models for maximum diameter, $D_{d}^{*}$, that is reached by the droplet. One is based on an energy conservation argument where the kinetic energy is converted to the surface energy. This model results in a scaling $D_{d}^{*} / D_{0} \propto \mathrm{We}^{1 / 2}$ [92]. The other more recent model balances the inertial force with the surface tension and suggests $D_{d}^{*} / D_{0} \propto \mathrm{We}^{1 / 4}[39]$. In the latter case significant amount of the impact energy is dissipated into internal degrees of the droplet.

In figure $2.6 a$, the maximum droplet diameter in our experiments is plotted against the Weber number We for different packing fractions. We observe that data with the same impact speed $U_{0}$ are scattered due to the variation in packing fraction, where a denser packing typically generates a larger droplet deformation. This can be understood from the dependence of the substrate deformation on the initial packing fraction (figure 2.5) together with equation 2.1, It has been shown that a denser packing deforms less, thus more energy is transferred into the droplet resulting in a larger spreading. In the energy conservation model [92], We needs to be replaced with an effective Weber number $\mathrm{We}^{\dagger}=D_{0} /\left(D_{0}+2 Z_{c}^{*}\right)$ We. Furthermore, during the impact the droplet experiences an average deceleration force with magnitude $\sim E_{d} / \frac{1}{2} D_{0}$ [equation 2.1]. Reproducing the argument in the force balance model introduced in ref. 39 , the droplet deforms to balance such a deceleration, which yields a scaling with $\mathrm{We}^{\dagger}$ as well. Therefore, we suggest to use $\mathrm{We}^{\dagger}=\frac{D_{0}}{D_{0}+2 Z_{c}^{*}}$ We for the impact on a deformable substrate, where $Z_{c}^{*}$ characterizes the deformation of the substrate.

When the maximum droplet deformations are plotted versus effective Weber number in figure $2.6 \mathrm{~b}$, we find that the data collapse onto a master curve. A power law fit gives $\mathrm{We}^{\dagger^{0.250 \pm 0.012}}$, which implies that our experiments are well described by the force balance model of Clanet et al. [39]. Previous studies on this topic rather used the traditional Weber number and reported smaller scaling exponents 
a)

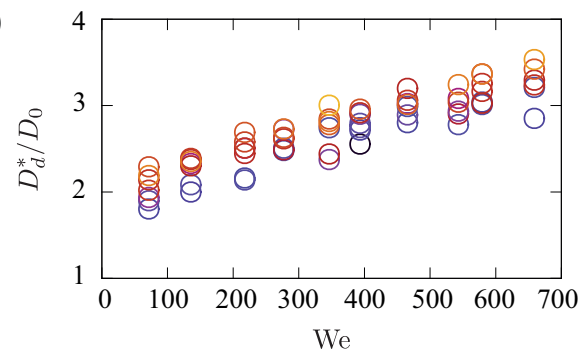

b)

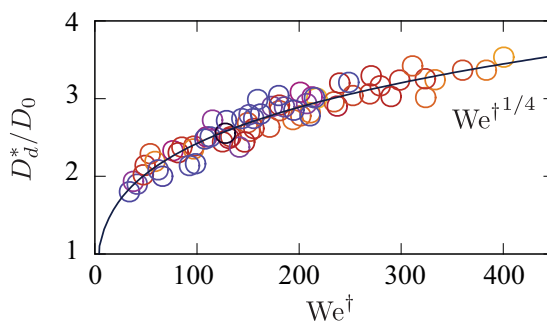

0.62

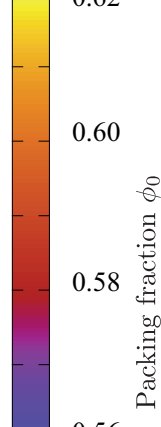

0.56

0.54

Figure 2.6: Maximum droplet deformation $D_{d}^{*}$ normalized with the initial droplet diameter $D_{0}$ versus Weber number We $=\rho_{l} D_{0} U_{0}^{2} / \sigma$ in $a$ ) and the effective Weber number $\mathrm{We}^{\dagger}=D_{0} /\left(D_{0}+2 Z_{c}^{*}\right)$ We in $b$ ), where $Z_{c}^{*}$ is the maximum crater depth (figure 2.3). Packing fraction is color coded. The dashed line in $b$ ) indicates $D_{d}^{*} / D_{0} \propto \mathrm{We}^{\dagger 1 / 4}$.

no larger than $1 / 5$ [15, 18]. This had been interpreted either as a viscous effect [18] or as a result of the density ratio of the liquid and sand [15]. However, liquids with the same viscosity and density but different surface tension scale differently [18]. We speculate that, other than viscosity, the impact energy distribution in equation 2.1 may be helpful to understand those inconsistent observations in the literature. The mechanism leading to equation 2.1 is that the impact energy $E_{k}$ is distributed according to the relative stiffness of the intruder and the target, i.e., the balance goes always towards the most easy deformation. The extreme case is that either the intruder or the target is undeformable, in which case the energy quickly transfers into the deformable medium. When both intruder and target are deformable, the impact energy dissipates more into the 'softer' one. For instance, while impacting on the same substrate, a liquid droplet with a smaller surface tension is easier to deform, and in consequence the substrate behaves more solid-like, and $\mathrm{We}^{1 / 4}$ is more likely to be recovered. This could explain the different scaling exponents found for liquids that only differ in surface tension in ref. 18. The computation of equation 2.1 requires the maximum crater depth, $Z_{c}^{*}$, which is measured for the first time in the present experiments. 


\subsection{Liquid-grain mixture and the crater morphology}

Until so far we considered the deformation of the target and that of the intruder separately. However, they are 'miscible' as well, i.e., the liquid that composes the droplet penetrates into the granular substrate. This penetration results in the formation of a liquid-grain mixture which needs to be taken into account in order to understand the crater morphology $[17,18,21]$. By changing impact speed $U_{0}$ and the initial packing fraction $\phi_{0}$ the crater morphology is found to vary systematically. According to the residue shape the observed craters are categorized into three groups: doughnut, truffle, and pancake (figure 2.1). The residue consists of a mixture of liquid and grains, which is referred to as 'liquid marble' in ref. 21. The discrimination between doughnut and truffle is vague, as while changing the impact parameters one shape seems to be continuously transformed into the other, whereas the transition from truffle to pancake is more abrupt. In this section, a model about the liquid penetration is established together with the knowledge already obtained in the previous sections. We will use this model to explain the various crater morphologies.

The doughnut and truffle residues are formed from the grains mixed with the liquid which are transported towards crater center by the droplet contraction. If the penetration during the impact is little, after the contraction, pure liquid concentrates at the crater center surrounded by the mixture of liquid and grains. After a few hundreds of milliseconds, the liquid in the center penetrates into the substrate due to gravity and capillary force leaving a dimple, i.e., creating a doughnut residue. More penetration results in a smaller amount of pure liquid at the center, such that the residue gradually becomes a truffle. With even more penetration the droplet is hardly able to contract, leaving a flat residue, the pancake shape. Therefore, knowing the amount of penetration of liquid between grains is crucial to understand the morphology phase diagram.

The transition from doughnut to truffle is continuous, while a sharper transition between truffle and pancake is observed. We estimate the volume of the liquid-grain mixture to characterize the penetration amount and define a threshold volume $V^{*}$ to separate doughnut/truffle and pancake regime. When this threshold mixture volume, $V^{*}$, is reached, the droplet loses the high curvature edge that promotes contraction. This happens when the volume of the mixture in the substrate, $V^{*}$, equals the volume of pure liquid above the substrate, $V_{l}-V^{*}(1-\phi)$, where $V_{l}$ is the initial droplet volume (figure 2.7 inset):

$$
V^{*}=\frac{V_{l}}{2-\phi}
$$




\section{CHAPTER 2. CRATER MORPHOLOGIES}

If the time scale to reach this critical mixing volume, $t_{\text {mix }}$, is shorter than the impact time scale $t_{\text {imp }}=\left(D_{0}+2 Z_{c}^{*}\right) / U_{0}$, the droplet contraction is suppressed, and pancake shapes are observed. Otherwise, the droplet is able to contract, mixed with grains, and forms a doughnut/truffle residue.

To quantitatively examine the above picture, we need to formulate the mixing progress. Describing the granular substrate as a porous medium, we start with Darcy's law:

$$
\frac{Q}{A} \approx \frac{\kappa P}{\mu L} .
$$

Here, the permeability of the granular packings, $\kappa$, is defined by Carman-Kozeny relation $\kappa=(1-\phi)^{3} d_{g}^{2} /\left(180 \phi^{2}\right)$, where $Q$ is the volume flux into the porous substrate, $P$ the driving pressure, $\mu$ the dynamic viscosity of the liquid, $L$ the penetration depth of the liquid into the porous substrate, and $A$ the contact area. In equation 2.3 we estimate the pressure gradient inside the sand as $P / L$. Furthermore, the conservation of liquid volume calls for

$$
\frac{Q}{A}=(1-\phi) \frac{d L}{d t} .
$$

Here, we only consider $L$ as a function of time. The total liquid volume penetrating into the substrate is given by $(1-\phi) A L$.

Combining equation 2.3 and equation 2.4, the penetration depth $L$ is solved as $L(t)=\sqrt{2 P \kappa /(\mu(1-\phi)) t}$. From here, one can define the time scale for which the volume of the mixture reaches the critical volume, $A L\left(t_{\mathrm{mix}}\right)=V^{*}$ :

$$
t_{\mathrm{mix}}=\left(\frac{V^{*}}{A}\right)^{2} \frac{(1-\phi) \mu}{2 \kappa P} .
$$

To apply equation 2.5 a few quantities need to be evaluated and explained. The contact area is estimated as $A=\pi D_{d}^{* 2} / 4$, i.e., the contact area at the measured maximum spreading diameter. The packing fraction $\phi$ is evaluated by the critical packing fraction $\phi^{*}$ rather than the initial packing fraction $\phi_{0}$ as explained in figure 2.4. The last missing piece is the driving pressure $P$. There are three candidates: the gravitational pressure, the inertial pressure, and the capillary pressure caused by the hydrophilicity of the grains that tries to pull the liquid into the bed. Since the droplet diameter is at the magnitude of the capillary length, gravity cannot significantly deform the droplet. The large droplet deformation shown in figure 2.6 indicates that the inertial pressure is much larger than gravity. A simple experiment of zero impact speed, in which we determine how fast the droplet is absorbed by the bed, also indicates that for the used grains capillary pressure is 


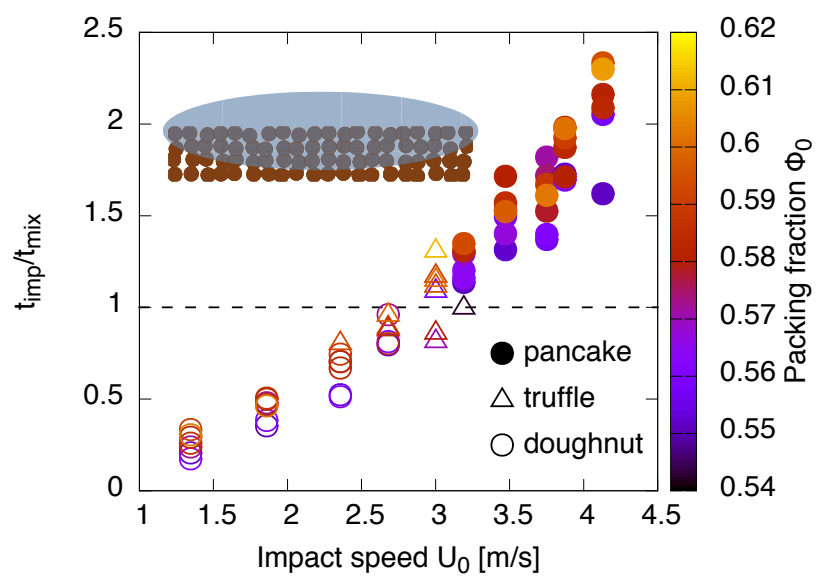

Figure 2.7: Phase diagram of the crater morphology. On the y-axis we plot the ratio between two time scales, namely the impact time scale $t_{\text {imp }}$ and the mixing time $t_{\text {mix }}$. The horizontal dashed line indicates a ratio of 1 , where the transition is expected. On the $x$-axis we have the impact speed $U_{0}$. The various crater morphologies are labeled with different symbols (see legend), whereas the color of the symbols indicate initial packing fraction. The inset shows a schematic of the droplet for which $V=V^{*}$ at maximum expansion.

at least two order of magnitude smaller than the inertial pressure. Therefore it is justified to consider the inertial pressure as the driving pressure. In the analysis of maximum droplet deformation and the introduction of the effective Weber number $\mathrm{We}^{\dagger}$ it was already implied that the average deceleration force experienced by the droplet equals $E_{d} / \frac{1}{2} D_{0}$ [equation 2.1]. The inertial pressure is evaluated in consequence as:

$$
P=\frac{E_{d}}{\frac{1}{2} D_{0} A}=\frac{M_{l} U_{0}^{2}}{A\left(D_{0}+2 Z_{c}^{*}\right)} .
$$

With all the quantities in equation 2.5 now defined, we can finally compare the time scales, $t_{\mathrm{imp}}$ and $t_{\mathrm{mix}}$, in figure 2.7 .

Three features from the experimental phase diagram are recovered in figure 2.7. (i) the transition is dominated by impact speed; (ii) a denser packing induces more mixture; and (iii) the expected transition discriminating doughnut/truffle and pancake morphology around $U_{0} \approx 3.2 \mathrm{~m} / \mathrm{s}$ is indeed indicated by the condition $t_{\mathrm{imp}}=t_{\mathrm{mix}}$. We emphasize that the inertial pressure driving penetration during the droplet spreading is considered to dominate the crater morphology transition, rather than gravity during the recession as was suggested in ref. 17 . Therefore, the reference time scale is the characteristic period of the inertial pres- 
sure, $t_{\text {imp }}$, rather than the contact time scale $\sim \sqrt{\rho_{l} D_{0}^{2} / \sigma}$ [21]. Finally, it is worthy to note that according to equation 2.5 and equation 2.6 the ratio of the time scales $t_{\text {imp }} / t_{\text {mix }}$ is independent of the substrate deformation $Z_{c}^{*}$. To apply and check the model, only $D_{d}^{*}$ needs to be measured.

\subsection{Conclusion}

In this chapter, we study the deformation of the droplet and the granular packing during impact. The deformation of the granular substrate decreases with the initial packing fraction (figure 2.5), however, the deformation speed exhibits evidence of dilation and defines a critical packing fraction $\phi^{*} \approx 0.585$ in figure 2.4 The substrate deformation introduces an impact energy distribution, equation 2.1 between the intruder and the target. An effective Weber number, $\mathrm{We}^{\dagger}$, is defined accordingly, and the droplet maximum deformation, $D_{d}^{*}$, is shown to be consistent with a scaling law $D_{d}^{*} \sim \mathrm{We}^{\dagger 1 / 4}$ in figure 2.6, which suggests a scenario where surface tension balances inertial pressure. Finally, based on the results of $\phi^{*}$ and $D_{d}^{*}$, a model is established to describe the penetration of the liquid into the substrate. This model evaluates the competition between the penetration time and the impact time, from which it is able to explain the observed morphology transition between the doughnut/truffle and the pancake crater shapes (figure 2.7. The energy distribution as given in equation 2.1, which is essential to understand the deformation of the target and the intruder, is estimated with the average interaction force. Its validation needs time resolved measurement of the interaction force. 


\section{Liquid-grain mixing suppresses droplet spreading and splashing}

$\|$

Would a raindrop impacting on a coarse beach behave differently from that impacting on a desert of fine sand? We study this question by a series of model experiments, where the packing density of the granular target, the wettability of individual grains, the grain size, the impacting liquid, and the impact speed are varied. We find that by increasing the grain size and/or the wettability of individual grains the maximum droplet spreading undergoes a transition from a capillary regime towards a viscous regime, and splashing is suppressed. The liquid-grain mixing is discovered to be the underlying mechanism. An effective viscosity is defined accordingly to quantitatively explain the observations.

This chapter is published as: Song-Chuan Zhao, Rianne de Jong, and Devaraj van der Meer, "Liquid-grain mixing suppresses droplet spreading and splashing during impact", Phys. Rev. Lett. 118, 054502 (2017). 


\subsection{Introduction}

Droplet impact has been studied over a century since the spark visualizations of Worthington [28, 93]. Owing to the development of experimental techniques and computation power, our knowledge about the dynamics of droplet impact upon a solid surface or a liquid pool has greatly improved [29-31]. In general, the dynamics, quantified by, e.g., the maximum spreading diameter and the splashing threshold, are governed by the interplay of three forces, namely those due to the viscosity, surface tension, and inertia of the impacting droplet. In accordance with which forces are dominant, two distinct regimes can be identified [39, 49].

In contrast, and despite of its ubiquity, droplet impact on sand did not attract much attention until recently $[14,15,17-19,21,94,95]$, and the underlying physics is still largely unexplored. There are at least two unique features about droplet impact on sand. One is the particular force response of a granular target which can be both solid-like and liquid-like [56]. The other is the possibility of mixing between liquid and grains which has been shown to be responsible to the formation of various crater morphologies $[14,17,21,94]$. These features add new dimensions to the parameter space of droplet impact phenomena, e.g., the properties of individual grains and the whole packing, and therefore present new challenges as well. Besides potential applications in environmental science and agriculture [96], revealing the role that these new parameters play provides a framework to test to what extent the concepts established for the conventional droplet impact phenomena may be applied. In this chapter, we report our experimental study of the effect of the wettability of individual grains and the grain size on droplet impact dynamics.

\subsection{Experimental methods}

In our experiments the impacting droplet is composed of either water or ethanol mixed with food dye (mass fraction $<2 \%$ ) for visualization purposes. The diameter of the water droplets, $D_{0}$, is fixed to $2.8 \mathrm{~mm}$ for most experiments and to $3.5 \mathrm{~mm}$ occasionally. The diameter of the ethanol droplets is, in general, fixed to $1.8 \mathrm{~mm}$ and to $2.5 \mathrm{~mm}$ occasionally. The impacting droplet is released from a nozzle above the substrate. The impact speed, $U_{0}$, reaches from $1.1 \mathrm{~m} / \mathrm{s}$ to $5.5 \mathrm{~m} / \mathrm{s}$ by altering the falling height. The target consists of a bed of beads which is prepared at a packing density in the range of $0.55-0.63$ by air fluidization and taps 1 . While the droplet deformation is visualized with a high-speed camera, at the same instance

\footnotetext{
${ }^{1}$ The range of packing fraction for glass and ceramic beads are $0.55-0.6$ and $0.58-0.63$ respectively.
} 
Table 3.1: Contact angles for water and ethanol, $\theta_{w}$ and $\theta_{e}$, respectively and grain size $d_{g}$ for the used granular materials.

\begin{tabular}{lccc} 
Material & $d_{g}[\mu \mathrm{m}]$ & $\cos \theta_{w}$ & $\cos \theta_{e}$ \\
\hline Silane-coated soda lime & 114,200 & $<0$ & - \\
Ceramic & $98,167,257$ & 0.3 & - \\
Piranha-cleaned ceramic & $98,167,257$ & $0.6-0.7^{2}$ & 1
\end{tabular}

the deformation of the substrate surface is measured by an in-house-built highspeed laser profilometer, see chapter 2

We used three types of wettabilities for beads of various sizes [cf. Table 3.1]: hydrophobic silane-coated soda lime, hydrophilic $\mathrm{ZrO}_{2}$ ceramic, and very hydrophilic $\mathrm{ZrO}_{2}$ ceramic cleaned with a piranha solution. The grain size, $d_{g}$, is represented by the mean of the size distribution which is measured under a microscope for a sample of more than 100 grains. The contact angle of both types of ceramic beads is measured by recording the penetration time after a droplet deposition on a packing of grains [97], and no penetration is observed for the silane-coated beads.

\subsection{Maximum droplet spreading}

It is well known that the rigidity of a granular substrate is very sensitive to its packing density, $\phi_{0}$ [66, 68, 88, 90]. In the previous chapter2we have discussed the dependence of the maximum droplet spreading diameter, $D_{d}^{*}$, on $\phi_{0}$ and have shown that it can be understood from the partition of the kinetic energy of the impacting droplet into the deformation of both the droplet and the substrate. This partition leads to replacing the Weber number, We $=\frac{\rho_{l} D_{0} U_{0}^{2}}{\sigma}$, which is used to describe droplet spreading when it is limited by surface tension $\sigma$, by an effective Weber number, $\mathrm{We}^{\dagger}=\frac{D_{0}}{D_{0}+2 Z_{c}^{*}}$ We, where $Z_{c}^{*}$ is the maximum vertical deformation of the substrate measured by the dynamic laser profilometry and $\rho_{l}$ is the liquid density. It has been shown that $\mathrm{We}^{\dagger}$ collapses the $D_{d}^{*}$ data for various packing densities (see chapter2 and appendix 3.A).

In figure 3.1, $D_{d}^{*}$ normalized by $D_{0}$ is plotted against the effective Weber number $\mathrm{We}^{\dagger}$ for various combinations of liquids, grain types and grain sizes. It comes as no surprise to see that $D_{d}^{*}$ increases with $\mathrm{We}^{\dagger}$, yet the large spread in figure 3.1

\footnotetext{
${ }^{2}$ Due to aging under exposure to the ambient air, the contact angle of cleaned ceramic beads varies, however, its value is measured after the experiments of each data set.
} 

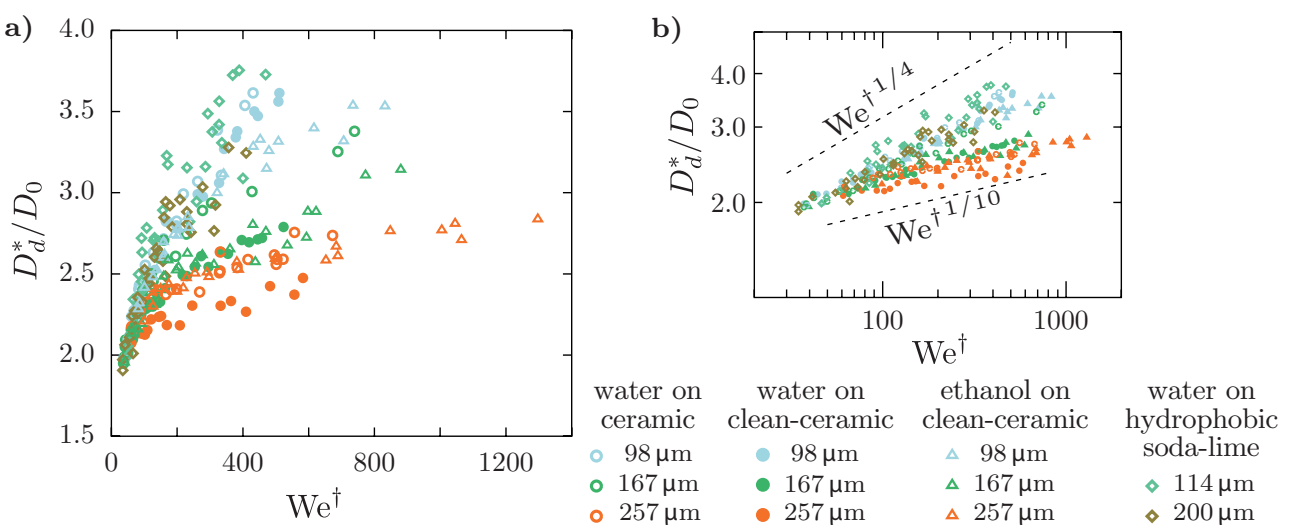

Figure 3.1: a) Maximum droplet spreading diameter $D_{d}^{*}$ scaled by the initial diameter of the droplet $D_{0}$ versus the effective Weber number $\mathrm{We}^{\dagger}$ (see the text for its definition). The results are plotted for different grain sizes (indicated by colors) and combinations of droplets and granular substrates (denoted by symbols). $b$ ) The same data is shown in logarithmic scale.

clearly indicates that $\mathrm{We}^{\dagger}$ alone is not sufficient to describe droplet spreading. Taking a closer look at the data set, four features can be distinguished:

i) The spreading diameter $D_{d}^{*}$ is suppressed with increasing grain size for any given combination of liquid and hydrophilic grain type (circles and triangles in the figure).

ii) For hydrophobic soda-lime beads, the grain size does not significantly affect $D_{d}^{*}$ (open diamonds).

iii) Water droplets impacting on the very hydrophilic ceramic grains result in smaller $D_{d}^{*}$ than those impacting on plain ceramic grains (open and solid circles).

iv) When plotted in the doubly logarithmic scale, the data appear to separate along two power laws: $\mathrm{We}^{\dagger^{1 / 4}}$ and $\mathrm{We}^{\dagger^{1 / 10}}$ (figure 3.1b).

In summary, these features indicate that the bulk wettability of the substrate affects $D_{d}^{*}$. This bulk wettability contains both the permeability of the substrate and the wettability of individual grains. The crucial question is therefore: how does the bulk wettability influence the relation between $D_{d}^{*}$ and $\mathrm{We}^{\dagger}$ ? Our investigation begins with a clue provided by the last listed feature. 
The two different power laws observed in figure $3.1 \mathrm{~b}$ imply different stopping mechanisms for droplet spreading. The impacts on hydrophobic grains and those on small hydrophilic grains behave as $D_{d}^{*} / D_{0} \propto \mathrm{We}^{\dagger 1 / 4}$ which indicates a force balance between inertia and surface tension [39, 94]. However, for the impacts on large hydrophilic grains we observe another type of scaling, namely close to $\mathrm{We}^{\dagger / 110}$. Such behavior is equivalent to $D_{d}^{*} / D_{0} \propto U_{0}^{1 / 5} \propto \mathrm{Re}^{1 / 5}$ which is a hallmark of the dominance of viscous dissipation [36, 39], where the Reynolds number, Re $=$ $U_{0} D_{0} / v_{l}$, stands for the significance of inertia relative to viscosity.

For a droplet impacting on a solid surface, the scaling $D_{d}^{*} / D_{0} \propto \mathrm{Re}^{1 / 5}$ can be understood as follows. While the droplet flattens during spreading, the thickness of the viscous boundary layer grows with time like $\sim \sqrt{v_{l} t}$, where $v_{l}$ is the kinematic viscosity of the liquid. If at the moment of maximum spreading the thickness of the liquid film, $\sim D_{0}^{3} / D_{d}^{* 2}$, matches that of the boundary layer, $\sqrt{v_{l} D_{d}^{*} / U_{0}}$, the spreading flow is stopped by viscosity, and one recovers the relation $D_{d}^{*} / D_{0} \propto$ $\mathrm{Re}^{1 / 5}[36$. It is plausible that the spread in figure 3.1 may be interpreted as a transition from a capillary regime to a viscous one. However, since the liquid viscosity, $v_{l}$, is virtually constant for all studied impacts, it is clear that the Reynolds number of the droplet is insufficient to explain such a transition. Nonetheless, the effect of the bulk wettability observed in figure 3.1 inspired us to regard the mixing between the liquid and grains as a boundary layer. In analogy to the viscous boundary layer, this mixing layer ceases liquid motion within it, due to strong viscous dissipation at the length scale of a grain. For hydrophobic grains the mixing is negligible, which explains that for those grains no grain size dependence of $D_{d}^{*}$ is observed. However, for hydrophilic grains the droplet spreading dynamics may well be altered. Therefore, to understand the two power laws shown in figure $3.1 b$ we analyze the development of the mixing layer.

\subsection{Effective viscosity}

We use Darcy's law to quantify the penetration flux of the impacting droplet into the substrate,

$$
\vec{Q}=\frac{\kappa A}{\mu_{l}} \vec{\nabla} P
$$

In the above equation the permeability of the substrate, $\kappa=(1-\phi)^{3} d_{g}^{2} /\left(180 \phi^{2}\right)$, is defined by the Carman-Kozeny relation [98], $\vec{\nabla} P$ is the pressure gradient, $A$ is the contact area between the droplet and the substrate, and $\mu_{l}=\rho_{l} v_{l}$ is the dynamic viscosity of the liquid. Since the pressure gradient is mainly in the vertical 
direction, equation 3.1 can be reduced to a scalar equation. The penetration of liquid into the substrate can now be viewed as the growth of a "boundary layer" into the droplet, whose thickness, $L$, is defined by its time derivative: $\mathrm{d} L / \mathrm{d} t=Q / A$. $L$ denotes the thickness of the liquid layer that merges with the sand, but due to the presence of the grains the penetration depth of the liquid into the sand bed is larger, namely $L /(1-\phi)$, and the pressure gradient can be estimated as $(1-\phi) P / L$. Equation 3.1 thus becomes an ordinary differential equation for the mixing layer thickness $L$ with respect to time $t$, and its solution is

$$
L(t)=\sqrt{\frac{2 \kappa P(1-\phi)}{\mu_{l}} t} .
$$

Besides the aforementioned physical analogy between the mixing layer and the viscous boundary layer, equation 3.2 indicates that the analogy extends to the mathematical form of the growth of their thicknesses as well, i.e., both are diffusive. Therefore, it can be used to define an effective viscosity, the quantity $v_{p} \equiv 2 \kappa P(1-\phi) / \mu_{l}$ that appears in front of $t$. While most quantities in equation 3.2 are merely properties of the substrate or the impacting liquid, the pressure $P$ that drives mixing is not. Therefore, estimating $P$ is the last remaining piece of the puzzle.

There are three potential sources of the driving pressure $P$ : inertia, capillarity and gravity. We estimate their orders of magnitude with typical parameters for the water droplets used in our experiments: liquid density $\rho_{l}=1.0 \times 10^{3} \mathrm{~kg} / \mathrm{m}^{3}$, surface tension $\sigma=72 \times 10^{-3} \mathrm{~N} / \mathrm{m}$, impact speed $U_{0} \sim 1-5 \mathrm{~m} / \mathrm{s}$, droplet diameter $D_{0} \approx 3 \mathrm{~mm}$, and grain size $d_{g} \sim 100 \mu \mathrm{m}$. Then one obtains a typical inertial pressure of $P_{i} \approx \rho_{l} U_{0}^{2} \sim 10^{3}-10^{4} \mathrm{~Pa}$, a capillary pressure of $P_{c} \approx 4 \sigma \cos \theta_{c} / d_{g} \sim 10^{3} \cos \theta_{c} \mathrm{~Pa}$, and a gravitational pressure of $P_{g} \approx \rho_{l} g D_{0} \sim 10 \mathrm{~Pa}$. For the liquids and hydrophilic grains that we used the contact angle stays in a range of $\cos \theta_{c} \in[0.3,1]$, hence, $P_{c}$ is at least one order of magnitude larger than $P_{g}$ which is therefore neglected. Though $P_{i}$ is again at least one order of magnitude larger than $P_{c}$, previous simulation and experimental works have shown that $P_{i}$ acts only within an inertial time scale $\tau_{i} \approx D_{0} / U_{0}[36,91]$. We correct this time scale as $\tau_{i}=\left(D_{0}+2 Z_{c}^{*}\right) / U_{0}$ by taking the deformation of the substrate, $Z_{c}^{*}$, into account. In contrast, $P_{c}$ lasts as long as the contact between liquid and grains exists. This contact time is estimated as half of the intrinsic oscillation time of the droplet [17, 99, 100], $\tau_{c}=\frac{1}{2} \sqrt{\frac{\pi}{6} \frac{\rho_{l} D_{0}^{3}}{\sigma}}$, and represents the time it takes until maximum droplet spreading is reached. Note that, in general, $\tau_{c}>\tau_{i}$. These two time scales provide relative weights for $P_{i}$ and $P_{c}$ in the spreading phase of the droplet, and the average effect of the total pressure is evaluated as $P=\frac{\tau_{i}}{\tau_{c}} P_{i}+P_{c}$, see appendix 3.B. Inserting this total pressure into 


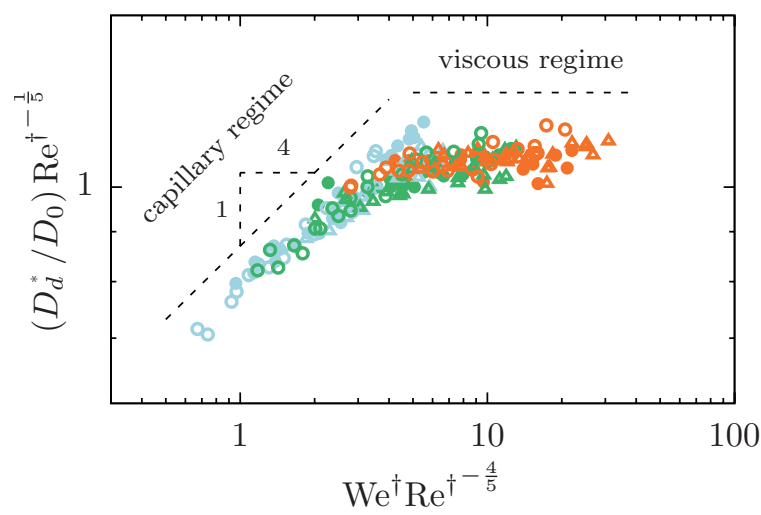

Figure 3.2: The maximum droplet spreading diameter, $D_{d}^{*} / D_{0}$, for all hydrophilic impacts of figure 3.1 in a doubly logarithmic plot. The same symbols and colors as in figure 3.1 are used. The data have been compensated in such a way that a transition between a capillary $\left(\propto \mathrm{We}^{\dagger 1 / 4}\right)$ and a viscous regime $\left(\propto \mathrm{Re}^{\dagger 1 / 5}\right)$ can be observed. The power laws of these two regimes are indicated by dashed lines.

equation 3.2 , the effective viscosity is estimated as

$$
v_{p}=\frac{2 \kappa(1-\phi)}{\mu_{l}} P=\frac{2 \kappa(1-\phi)}{\mu_{l}}\left(\frac{\tau_{i}}{\tau_{c}} P_{i}+P_{c}\right) \text {, }
$$

and a corresponding effective Reynolds number, $\operatorname{Re}^{\dagger}=U_{0} D_{0} / v_{p}$, is defined

When evaluating $v_{p}$, the inertial pressure (as in chapter 2) is corrected by the deformation of the substrate $Z_{c}^{*}, P_{i}=\rho_{l} U_{0}^{2} \frac{D_{0}}{D_{0}+2 Z_{c}^{*}}$; the capillary pressure is given by $P_{c}=4 \sigma \cos \theta_{c} / d_{c}$, where $d_{c}=\frac{2(1-\phi)}{3 \phi} d_{g}$ is the average diameter of capillaries between grains derived from the Carman-Kozeny relation; and the critical packing fraction of dilatancy, $\phi^{*}=0.59$, is used for all packings during impact (see [68], chapter 2 and appendix 3.B. We then find that $v_{p}$ is in the range of $10^{-5}-10^{-4}$ $\mathrm{m}^{2} / \mathrm{s} 3$, i.e., at least one order of magnitude larger than the kinematic viscosity of water, $v_{l}$. As a consequence the viscous boundary layer inside the droplet can be neglected. It is worthy to point out that $v_{p}$ could be smaller than $v_{l}$ when using parameters out of the range studied here, e.g., using highly viscous liquids and/or very small grain size, where the viscous boundary layer is likely to become dominant in turn.

\footnotetext{
${ }^{3}$ The magnitude of the resultant effective 'boundary' layer thickness, $L=\sqrt{v_{p} \tau_{c}}$, is $\sim 10^{-4} \mathrm{~m}$, and the actual mixing layer thickness, $L /(1-\phi)$, is in the range of $10^{-4}-10^{-3} \mathrm{~m}$.
} 
The effective viscosity defined in equation 3.3 grows with increasing grain size, $d_{g}$, on which it depends through $\kappa$ and $P_{c}$. In consequence, for large $d_{g}$ the droplet spreading is more likely to be stopped by liquid-grain mixing before surface tension can do so, and hence $D_{d}^{*} / D_{0} \propto \mathrm{Re}^{\dagger^{1 / 5}}$ would be expected. In contrast, for small $d_{g}$ mixing is slower and the surface tension balances inertia, leading to $D_{d}^{*} / D_{0} \propto \mathrm{We}^{\dagger 1 / 4}$. To illustrate the transition between these two scaling relations, data of all hydrophilic impacts are plotted as $D_{d}^{*} / D_{0} \mathrm{Re}^{\dagger^{-1 / 5}}$ versus $\mathrm{We}^{\dagger} \mathrm{Re}^{\dagger^{-4 / 5}}$ in figure 3.2 [39]. The newly introduced $\mathrm{Re}^{\dagger}$ successfully collapses data of various surface tensions, grain sizes, and wettabilities on a master curve without free parameters. Further discussion on the scaling laws can be found in appendix 3.C.

Leaving the mathematical details aside here (see appendix [3.B), the transition in figure 3.2 can be interpreted as a crossover from a regime where $D_{0}$ is the dominant length scale to one where both $D_{0}$ and $d_{g}$ matter, which, since $d_{g} \ll D_{0}$, implies that viscous dissipation in the mixing layer becomes important. This happens when $v_{p}$ is large, i.e., $\mathrm{Re}^{\dagger}$ is small. Previous studies about droplet spreading on sand have used the traditional Weber number and reported various scaling relations [15, 18, 19]. The introduction of $\mathrm{We}^{\dagger}$ and $\mathrm{Re}^{\dagger}$ (cf. figure 3.2), which take the deformability and bulk wettability of the substrate into account respectively, may provide a universal framework to understand droplet spreading when impacting on sand or other porous media.

\subsection{Splashing suppression}

With increasing impact velocity the inertia of the spreading liquid may overcome both surface tension and viscosity, and splashing can occur. Therefore, for an impact of droplets on solid substrates at a given Weber number, the Reynolds number determines whether a droplet will splash or not [49]. Is the same true for the effective Reynolds number, $\mathrm{Re}^{\dagger}$, introduced here? As the effective viscosity $v_{p}$ increases with $d_{g}$, resulting in a smaller $\mathrm{Re}^{\dagger}$, large grains are expected to suppress the splash. Indeed, as shown in figure 3.3, an ethanol droplet already splashes for $\mathrm{We}^{\dagger}=431$ when impacting on ceramic beads of $d_{g}=98 \mu \mathrm{m}$, whereas when impacting on the same grain type but with $d_{g}=257 \mu \mathrm{m}$, splashing is delayed until $\mathrm{We}^{\dagger}>6524$. To quantify the splashing threshold, Mundo, Sommerfeld and Tropea [49] proposed a dimensionless splashing parameter, $K_{d}=\mathrm{We}^{1 / 2} \mathrm{Re}^{1 / 4}$ relating inertial force to viscous and surface tension forces. Here, we replace the We-

\footnotetext{
${ }^{4}$ The splashing considered here consists of fragments expelled from a fully developed droplet rim rather than the prompt splashing which happens at the very early stage of the impact.
} 


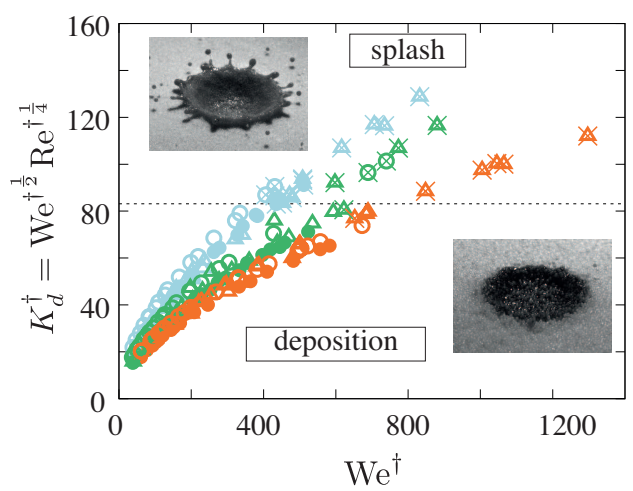

Figure 3.3: The splashing parameter $K_{d}^{\dagger}=\mathrm{We}^{\dagger^{1 / 2}} \mathrm{Re}^{\dagger^{1 / 4}}$ as a function of $\mathrm{We}^{\dagger}$. Here, $\mathrm{We}^{\dagger}$ and $\mathrm{Re}^{\dagger}$ are the effective Weber and Reynolds number, respectively, as defined in the text. The same colors and symbols as in figure 3.1 are used to denote various combinations of liquid and wettability of grains, while those impacts resulting in splashing/fragmentation are highlighted with a cross. The dashed line marks the threshold separating deposition and splashing regimes. The top left and bottom right insets show examples of a water droplet with $D_{0}=2.8 \mathrm{~mm}$ and $U_{0}=5.2 \mathrm{~m} / \mathrm{s}$ impacting on clean ceramic beads with $d_{g}=$ $98 \mu \mathrm{m}$ and ceramic beads with $d_{g}=257 \mu \mathrm{m}$ respectively.

ber and Reynolds numbers by their effective counterparts in the definition of $K_{d}$, which leads to $K_{d}^{\dagger}=\mathrm{We}^{\dagger 1 / 2} \mathrm{Re}^{\dagger / 1 / 4}$. A transition can be seen around $K_{d}^{\dagger} \approx 85$ for all hydrophilic impacts in figure 3.3, see appendix 3.D on the usage of the modified versus normal Reynolds number. It is necessary to point out that since the definition of $K_{d}$ is insensitive to substrate properties such as wettability and roughness [101, 102], the value of the splashing threshold differs from one situation to another, e.g., different values of $K_{d}=57.7,80$, and 120 are reported for impacts on a solid surface [49], nanofibers [50], and dry granular packings [18] respectively. Therefore, the threshold value reported here is not intended to be compared directly with the above-mentioned ones. Nevertheless, the existence of a unified splashing threshold for impacts on different grain sizes is another manifestation of how liquid-grain mixing is captured by $\mathrm{Re}^{\dagger}$.

\subsection{Discussion}

In this chapter we introduced effective Weber and Reynolds numbers $\mathrm{We}^{\dagger}$ and $\mathrm{Re}^{\dagger}$, which incorporate the deformability and bulk wettability of a granular substrate respectively. This reveals the hidden similarities between droplet impact 
on sand and that on a solid substrate for two aspects: maximum droplet spreading and splashing. Despite the similarities represented by $\mathrm{We}^{\dagger}$ and $\mathrm{Re}^{\dagger}$, there are distinctions resulting from the characteristics of a sand bed. One example stems from the mobility of individual dry grains which can result in a shear band under external driving [56]. It is thus plausible that, when mixing between liquid and grains is subtle, the boundary condition experienced by a spreading droplet on sand is neither purely slip nor no slip but one with a finite slip length [103] with the magnitude of the grain size. Another example is the role of ambient air. Owing to recent development of high-speed imaging techniques, ambient air is found to be responsible for splashing [46, 47] and bubble entrapment [104-107]. In contrast, the permeability of a sand bed may prevent the existence of such a thin air film. This also differentiates splashing suppression in figure 3.3 from that on deformable substrates [108]. Further work is necessary to understand the role of these unique features of a sand bed on the impact dynamics. 


\section{A Effective Weber number}

During impact, the kinetic energy of the droplet is transferred into deformations of both droplet and granular substrate. The partition of the impact energy depends on the stiffness of the droplet (surface tension) and the granular target, and latter is a function of its packing density, $\phi_{0}$. This energy partition can be estimated with the measured maximum crater depth, $Z_{c}^{*}$ as follows, where the crater depth is measured by laser profilometry in experiments.

The droplet experiences a deceleration force. This force does work on both the droplet and the granular target along the total displacement, $\frac{1}{2} D_{0}+Z_{c}^{*}$, where $D_{0}$ is the initial droplet diameter. Out of the total displacement, $\frac{1}{2} D_{0}$ is the contribution of the droplet deformation, while $Z_{c}^{*}$ is that of the substrate deformation. Such a force, $E_{k} /\left(\frac{1}{2} D_{0}+Z_{c}^{*}\right)$, indicates the average interaction between droplet and the substrate, from which we can estimate the energy absorbed by the droplet $E_{d}$ and that absorbed by the substrate $E_{s}$ as the work done by this interaction force to deform the droplet and substrate respectively:

$$
E_{d}=\frac{D_{0}}{D_{0}+2 Z_{c}^{*}} E_{k} ; E_{s}=\frac{Z_{c}^{*}}{D_{0} / 2+Z_{c}^{*}} E_{k}
$$

An effective Weber number is thus defined as $\mathrm{We}^{\dagger}=\frac{E_{d}}{\sigma D_{0}{ }^{2}}=\frac{D_{0}}{D_{0}+2 Z_{c}^{*}}$ We, a fraction of the normal Weber number. As shown in the previous chapter, $\mathrm{We}^{\dagger}$ collapses the maximum spreading diameter of the droplet for different $\phi_{0}$. It can also be seen in figure 3.4 for the data of water droplet impact on ceramic beads used in the main text.

\section{B The effective viscosity}

We used Darcy's law to model the penetration flux of the liquid into the granular substrate. The liquid mixed with grains are considered to be captured in a 'boundary layer', and the thickness of this boundary layer is denoted by $L$ (see figure 3.5 and main text). As a result, $L$ grows with time as

$$
\frac{\mathrm{d} L^{2}}{\mathrm{~d} t}=\frac{2 \kappa(1-\phi)}{\mu_{l}} P(t)
$$

Here, $\kappa=(1-\phi)^{3} d_{g}^{2} /\left(180 \phi^{2}\right)$ is the permeability defined by the Carman-Kozeny relation; $\mu_{l}$ is the dynamic viscosity of the liquid; $\phi$ is the packing density of the granular target; and $d_{g}$ is the grain size. The driving pressure, $P(t)$, consists of 

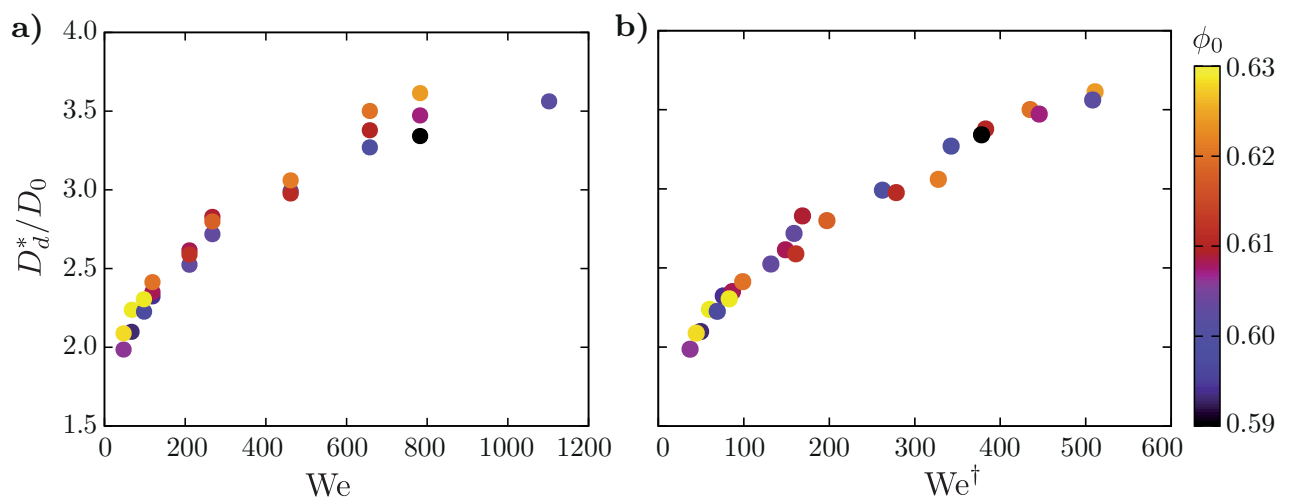

Figure 3.4: The maximum spreading diameter, $D_{d}^{*}$, versus the traditional Weber number, We, $a$ ) and the effective Weber number, $\mathrm{We}^{\dagger}, b$ ) for water droplet impact on piranha cleaned ceramic beads (bead diameter $=98 \mu \mathrm{m}$ ). Color represents initial packing density of the granular target. Clearly, $\mathrm{We}^{\dagger}$ collapse the data better than We.

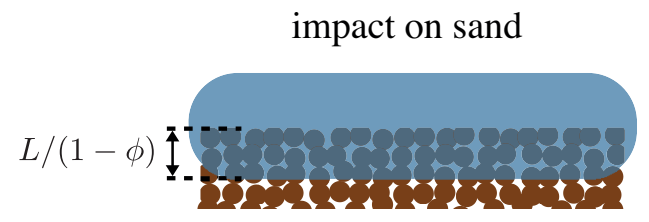

impact on solid surface

Figure 3.5: The mixing layer (left) is regarded as an analogy of the viscous boundary layer of a droplet impacting on solid surface (right). The actual thickness of the mixing layer is defined as $L /(1-\phi)$ (see main text).

two parts: the inertial and the capillary pressure, $P_{i}$ and $P_{c}$. These two pressures are different in both intensity and duration, see the sketch in figure 3.6, $P_{i}$ only acts during the time scale $\tau_{i}=\left(D_{0}+2 Z_{c}^{*}\right) / U_{0}$, while $P_{c}$ acts as long as the liquidgrain interface exists. Herein we only consider the dynamics within the droplet spreading phase the duration of which is estimated as $\tau_{c}=\frac{1}{2} \sqrt{\frac{\pi}{6} \frac{\rho_{l} D_{0}^{3}}{\sigma}}$, i.e., half of the intrinsic oscillation time. The total pressure can thus be written as: $P(t)=$ $P_{i} \not{H}\left(1-t / \tau_{i}\right)+P_{c}$ for $t \leq \tau_{c}$, where $\mathscr{H}$ is the Heaviside step function.

Integrating equation 3.5 over a time $t$ of the order of $\tau_{c}>\tau_{i}$ gives the result that $L^{2}(t)$ is a linear combination of $P_{i}$ and $P_{c}$

$$
L^{2}(t)=\frac{2 \kappa(1-\phi)}{\mu_{l}}\left(\frac{\tau_{i}}{\tau_{c}} P_{i}+P_{c}\right) t .
$$

The time scales $\tau_{i}$ and $\tau_{c}$ therefore represent the relative weight of $P_{i}$ and $P_{c}$, and the average effect of the total pressure during the droplet spreading phase is thus 


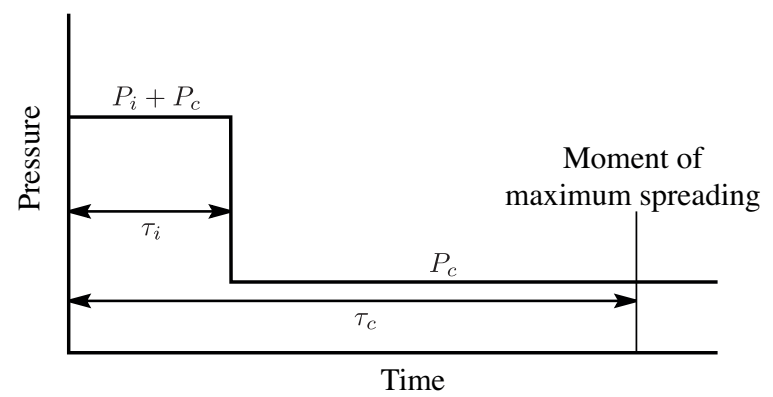

Figure 3.6: A sketch of the relative intensity of inertial and capillary pressure, $P_{i}$ and $P_{c}$, against time.

$\left(\frac{\tau_{i}}{\tau_{c}} P_{i}+P_{c}\right)$. The corresponding effective viscosity appears in front of $t$

$$
v_{p}=\frac{2 \kappa(1-\phi)}{\mu_{l}}\left(\frac{\tau_{i}}{\tau_{c}} P_{i}+P_{c}\right) .
$$

The ingredients of equation 3.7 may now be evaluated as follows (see main text)

$$
\begin{aligned}
P_{i} & =\frac{D_{0}}{D_{0}+2 Z_{c}^{*}} \rho_{l} U_{0}^{2} ; & P_{c} & =\frac{4 \sigma \cos \theta_{c}}{d_{c}}=\frac{6 \phi}{1-\phi} \frac{\sigma \cos \theta_{c}}{d_{g}} ; \\
\tau_{i} & =\frac{D_{0}+2 Z_{c}^{*}}{U_{0}} ; & \tau_{c} & =\frac{1}{2} \sqrt{\frac{\pi}{6} \frac{\rho_{l} D_{0}^{3}}{\sigma}},
\end{aligned}
$$

with which equation 3.7 can now be explicitly written in the experimental parameters

$$
\begin{array}{r}
v_{p}=\frac{1}{\mu_{l}}\left(A(\phi) \sqrt{\frac{\sigma \rho_{l} U_{0}^{2}}{D_{0}}} d_{g}^{2}+B(\phi) \sigma \cos \theta_{c} d_{g}\right), \\
\text { with } A(\phi)=\sqrt{\frac{6}{\pi}} \frac{(1-\phi)^{4}}{45 \phi^{2}} \text { and } B(\phi)=\frac{(1-\phi)^{3}}{15 \phi} \text {. }
\end{array}
$$

According to equations 3.6 and 3.7 $v_{p}$ is the diffusion rate of the liquid penetration into the substrate, and the first and second term are the contribution from inertial and capillary pressure respectively. It is noteworthy that the resultant $v_{p}$ in equation 3.8 is independent of the correction of the deformation of the sand bed, $Z_{c}^{*}$, and depends only on experimental parameters and material properties. According to our previous chapter, the granular bed just below the impacting droplet 
arrange itself towards $\phi^{*}=0.59$ during impact. This is the critical packing fraction of dilation which is found in solid ball impact experiments [68] as well. It is the maximal packing fraction grains can have when an object is moving through it. More dilute packings will be (locally) compressed to $\phi^{*}$, whereas more dense packings will dilate to $\phi^{*}$. We thus choose the critical packing fraction for all impacts, $\phi=\phi^{*}$, and therefore, $A(\phi), B(\phi)$ are constants in the following 5 . The corresponding effective Reynolds number, which stands for the ratio of advection rate due to the droplet spreading, $U_{0} D_{0}$, and diffusion rate, $v_{p}$, is

$$
\begin{aligned}
\operatorname{Re}^{\dagger} & =\frac{U_{0} D_{0}}{v_{p}}=\frac{1}{A\left(\frac{d_{g}}{D_{0}}\right)^{\frac{3}{2}} \frac{\sqrt{\sigma \rho_{l} d_{g}}}{\mu_{l}}+B \frac{\sigma \cos \theta_{c} d_{g}}{U_{0} D_{0} \mu_{l}}} \\
& =\frac{1}{\frac{A}{\mathrm{Oh}}\left(\frac{d_{g}}{D_{0}}\right)^{2}+\frac{B}{\mathrm{Ca}} \cos \theta_{c}\left(\frac{d_{g}}{D_{0}}\right)} .
\end{aligned}
$$

Here, the Ohnesorge and Capillary number are defined as $\mathrm{Oh}=\mu_{l} / \sqrt{\sigma \rho_{l} D_{0}}$ and $\mathrm{Ca}=U_{0} \mu_{l} / \sigma$. The ratio of the two terms in the denominator in equation 3.9 is $\eta \approx 0.32 \frac{\mathrm{We}^{1 / 2}}{\cos \theta_{c}} \frac{d_{g}}{D_{0}}$, where $\mathrm{We}=\frac{\rho_{l} D_{0} U_{0}^{2}}{\sigma}$ is the Weber number. If $\eta \ll 1$, the second term dominates over the first term, and the diffusion rate $v_{p}$ is governed by the capillary pull, such that $\operatorname{Re}^{\dagger} \approx \frac{\mathrm{Ca} D_{0}}{B \cos \theta_{c} d_{g}}$. Otherwise, if $\eta \gg 1$, the capillary effect is negligible, and $\mathrm{Re}^{\dagger}$ approaches the asymptotic value $\frac{\mathrm{Oh}}{A}\left(\frac{D_{0}}{d_{g}}\right)^{2}$ which is noticeably independent of the impact speed $U_{0}$, because the influence of the impact velocity in the advection rate (of droplet spreading) and that in the diffusion rate (of liquid penetration caused by inertial pressure) cancel each other out. In between these two extremes in $\eta$, both terms need to be taken into account. In our experiments, for a water droplet of $D_{0} \approx 3 \mathrm{~mm}$, the Weber number is between 42 and 1043, and the contact angle stays in a range of $\cos \theta_{c} \in[0.3,1]$. Therefore, $\eta \sim \mathscr{O}(1)$ for most impacts except that of small $U_{0}$ and hydrophilic ceramic beads of $d_{g}=98 \mu \mathrm{m}$. Our experiments are thus in the regime where both inertial pressure and capillary suction are important.

\footnotetext{
${ }^{5}$ Note that $A(\phi)$ and $B(\phi)$ are slowly varying functions of $\phi$ anyway, so one may take them constant in the range of $\phi$ used here.
} 

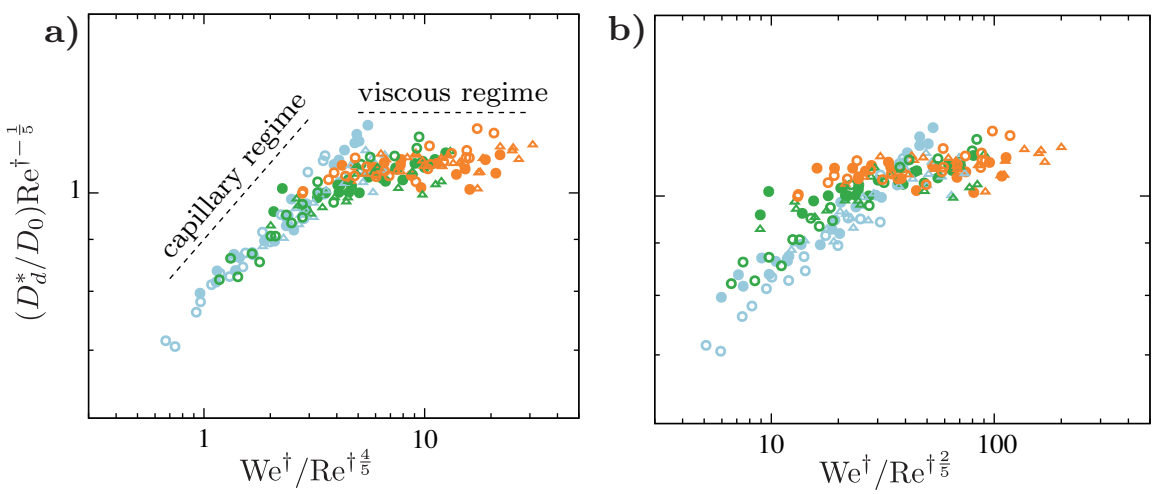

Figure 3.7: The maximum spreading diameter of the droplet, $D_{d}^{*}$, normalized by its initial diameter, $D_{0}$, for all impact on hydrophilic grains. The symbols and colors are the same as in figure 3.1 $a$ ) and $b$ ) show the same data but against different exponent of $\operatorname{Re}^{\dagger}$. See the main text for the definition of $\mathrm{We}^{\dagger}$.

\section{C The transition from the capillary regime to the viscous regime}

A transition of the maximum droplet spreading diameter, $D_{d}^{*}$, is expected from the capillary regime to the viscous regime by increasing $d_{g}$, which decreases $\operatorname{Re}^{\dagger}$. In the viscous regime, a scaling of $D_{d}^{*} / D_{0} \propto \mathrm{Re}^{\dagger^{1 / 5}}$ is predicted (see Sec. 3.4), and in the capillary regime $D_{d}^{*} / D_{0}$ follows a scaling of $\mathrm{We}^{\dagger}{ }^{\alpha}$. Whether the droplet spreading is in the capillary regime or in the viscous regime is determined by the relative significance of the scaling laws in these two regimes, i.e., $\mathrm{We}^{\dagger^{\alpha}} / \mathrm{Re}^{\dagger^{1 / 5}}$. The transition between them occurs, when $\mathrm{We}^{\dagger^{\alpha}} / \mathrm{Re}^{\dagger^{1 / 5}} \sim \mathscr{O}(1)$. Therefore, the scaling exponent for the capillary regime, $\alpha$ in $\mathrm{We}^{\dagger}{ }^{\alpha}$, has some consequence for the location of the transition point.

For the capillary regime, it is suggested that $D_{d}^{*} / D_{0} \propto \mathrm{We}^{\dagger^{1 / 4}}$ by the momentum balance model [39]. On the other hand, from the perspective of the total energy balance analysis for drop impact on a solid surface [32-35, 38], $D_{d}^{*} / D_{0}$ can not be described by a single scaling law of the Weber number, and recently a broad crossover between $\mathrm{We}^{1 / 2}$ and $\mathrm{Re}^{1 / 5}$ is proposed, implying that $D_{d}^{*} / D_{0}$ is a function of $\mathrm{We}^{1 / 2} / \mathrm{Re}^{1 / 5}$ [37]. We plot our data with both $\alpha=1 / 2$ and $\alpha=1 / 4$ in figure 3.7 . Note that in both figures the vertical axis are the same, and the plateau representing the viscous regime is close to 1 , which indicates that $\operatorname{Re}^{\dagger}$ captures the droplet spreading dynamics in this regime quantitatively. However, the two figures differ 

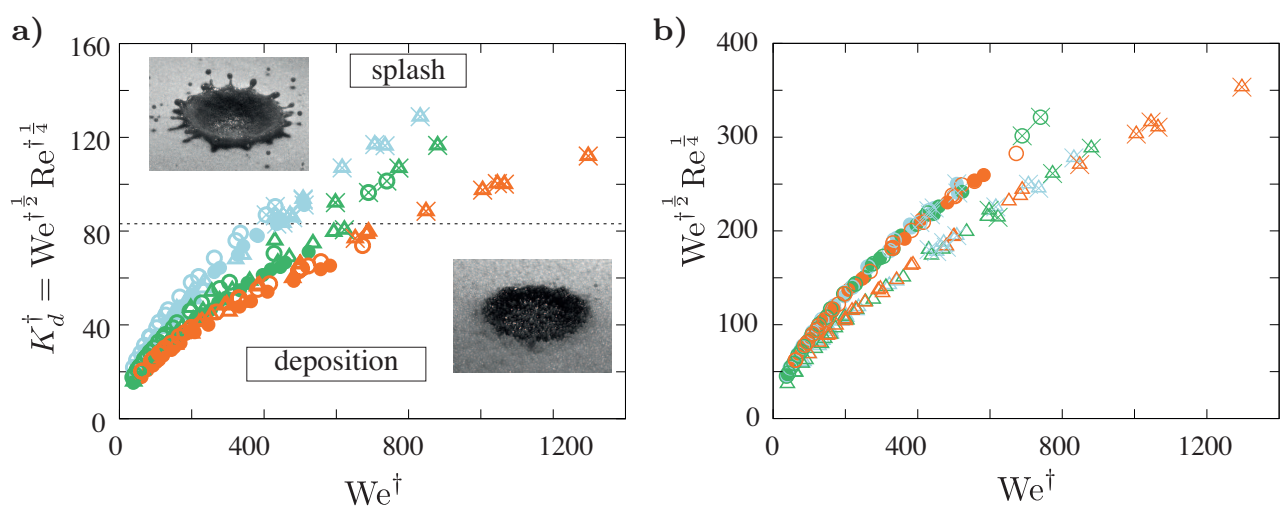

Figure 3.8: $a$ ) This figure is the same as figure 3.3 in the main text. $b$ ) The same data is plotted, but on the vertical axis the traditional Reynolds number is used. No splashing threshold can be identified for this plot.

in the exponent of $\mathrm{Re}^{\dagger}$ in their abscissa axis, which stems from different scalings of $\mathrm{We}^{\dagger}$ in the capillary regime. Our data fit the first mentioned way better (figure $3.7 a$ which is the same as figure 3.2). Furthermore, the $\mathrm{We}^{1 / 4}$ scaling has been reported in various impact experiments [39, 45, 94] and has been realized for We $>30$ using a more detailed energy balance model [44]. In conclusion, we find that the power law $D_{d}^{*} / D_{0} \propto \mathrm{We}^{\dagger 1 / 4}$ most accurately describes the capillary regime for the range of parameters studied in our experiments.

\section{D Splashing threshold}

The effective Reynolds number, $\mathrm{Re}^{\dagger}$, is also used to explain droplet splashing. With $\mathrm{Re}^{\dagger}$, a splashing threshold, $K_{d}^{\dagger}=\mathrm{We}^{\dagger^{1 / 2}} \mathrm{Re}^{\dagger 1 / 4} \approx 85$, can be defined (cf. figure 3.8 a). However, with the traditional Reynolds number no clear threshold can be identified (figure $3.8 b$ ). For a large range of values of $\mathrm{We}^{\dagger 1 / 2} \mathrm{Re}^{1 / 4}$ both splashes and impacts without splash occur. 


\section{4 \\ Crater formation during impact}

॥

After a raindrop impacts on a granular bed, a crater is formed as both drop and target deform. After an initial, transient, phase in which the maximum crater depth is reached, the crater broadens outwards until a final steady shape is attained. By varying the impact velocity of the drop and the packing density of the bed, we find that avalanches of grains are important in the second phase and hence, affect the final crater shape. In chapter 2, we introduced an estimate of the impact energy going solely into sand deformation and here we show that both the transient and final crater diameter collapse with this quantity for various packing densities. The aspect ratio of the transient crater is however altered by changes in the packing fraction.

This chapter is published as: Rianne de Jong, Song-Chuan Zhao, and Devaraj van der Meer, "Crater formation during raindrop impact on sand", Phys. Rev. E 95, 042901 (2017). 


\subsection{Introduction}

The impact of a droplet on a bed of sand or soil is amongst the most common events in nature; it is observed in agriculture, where rain is needed for proper crop growth; and it occurs in industry in the process of wet granulation which is the basis of the production of many pharmaceuticals. The rich phenomenon of drop impact on granular matter has only started to draw attention recently $14-18,20$, 21, 86, 94, 109, 110] and its underlying physics is still largely unrevealed. One of these aspects that is not yet fully understood, is the formation of impact craters during droplet impact, which is the focus of this work.

In the literature, craters formed by solid intruders have been observed from small laboratory to very large scale geological events, where the development and shape of these (planetary) craters continuously intrigued the scientific field for more than 30 years $[70,71,79,81-83,87$, 111, 112]. Melosh [70] divided the evolution of a geological crater into three phases: first, the initial contact, almost instantly followed by a shock wave through the granular bed, second, the transient crater growth and, third, slumping of the crater walls and/or collapse of the splash (or jet) at the center [70, 71]. These three phases have also been observed in laboratory experiments for solid intruder impact on dry grains $[61,73,81,82,87,112]$. If impact energy is lowered, if gravitational energy is increased or if the target is harder to permeate, the third phase may not occur [79] and, for dry grains, be replaced by avalanches.

Impact of droplets on grains results in a very different type of complex behavior. Observations for single drop impact on (dry) grains, show that both intruder and target deform, and mixing occurs (for wettable grains) $14-18,20,21,86,94$, 110], and recently an analogy between the raindrop imprints and asteroid strikes was drawn [21]. In contrast to solid ball impact on sand, droplet deformation also consumes part of the kinetic energy $E_{k}$ of the impactor, and the energy partition depends on the stiffness of both intruder and target (chapters 2 and 3). We will show that the deformability of the intruder can significantly affect crater formation. This is consistent with what is found for deformable granular projectiles impacts on sand [113].

The sand bed response after drop impact is mainly investigated by means of determining the final crater diameter, for which various scaling laws are obtained [14, 16-18, 21]. The maximum depth of the crater usually cannot be measured from the craters final profile as the irregularly shaped liquid-grain residue obstructs it, but it may be obtained in the cases where the droplet (or a mixture of droplet and grains) rebounces [21]. In chapter 2, however, high-speed laser profilometry during impact is used to acquire the transient behavior of the crater 
depth.

In this work we study both the transient and final crater shape, focusing on the evolution from the moment in time $t^{*}$ at which the maximum crater depth is reached until the crater obtains a final steady shape (i.e., at some point in time $t^{\infty}$ long after the impact). In particular, we will show that avalanches play a key role in the post-impact evolution of the crater, which is a mechanism that is not traditionally accounted for in the literature. Although here we focus on deformable intruders, our results are expected to be of relevance as well for, e.g., solid intruders. In addition, we study the effect of both the initial drop energy $E_{k}$ and the packing density of the bed. The structure of this chapter is as follows: We will first discuss the experimental method (Sec.4.2), after which we start the result section, Sec. 4.3 with discussing the final crater shape and how it depends on the impact parameters. It is followed by the crater evolution (Sec.4.3.3), from the perspectives of the slope of the crater and the displaced volume. Finally, we review and compare our results with previous works in Sec. 4.4, where various scaling laws will be discussed in more detail.

\subsection{Experimental method}

The intruder, an ultrapure (milli-Q) water droplet (density $\rho_{l}=998 \mathrm{~kg} / \mathrm{m}^{3}$, surface tension $\sigma=73 \mathrm{mN} / \mathrm{m}$, viscosity $\mu=1.0 \mathrm{mPa} / \mathrm{s}$ ), is released from a needle when gravity overcomes surface tension, resulting in a droplet with a diameter of $D_{0}=$ $2.84 \pm 0.03 \mathrm{~mm}$. The flow rate of the syringe pump is $0.15 \mathrm{ml} / \mathrm{min}$, which is sufficiently small to minimize inertial effects and obtain a reproducible droplet size. The droplet is released from various heights, giving it an impact speed $U_{0}$ varying between $1.3-4.2 \mathrm{~m} / \mathrm{s}$, which is determined from a calibrated height-impact speed curve. As target, a granular bed of at least $23 \mathrm{~mm}$ in height and $39 \mathrm{~mm}$ in diameter is used, consisting of polydisperse glass beads with a diameter $d_{g}=70-110 \mu \mathrm{m}$ and specific density $\rho_{g}=2.5 \cdot 10^{3} \mathrm{~kg} / \mathrm{m}^{3}$. Before the experiments, the beads are dried in the oven for at least half an hour. The bed is carefully prepared by air fluidization and optionally compacted with a tapping device (figure $4.1 \mathrm{a}$ ) to reach a variety of packing fractions between $0.54<\phi_{0}<0.61$. The latter is calculated by means of weighing the grains in combination with measuring the surface height before impact relative to the container rim (along the line of major height variation, i.e., the direction of tapping).

We study the effect of droplet impact on grains during as well as after the impact. To obtain both horizontal and vertical substrate deformation, we shine a laser sheet (with a depth of focus of $20.4 \mathrm{~mm}$ and width of $79 \mu \mathrm{m}$ ) across the gran- 
a)

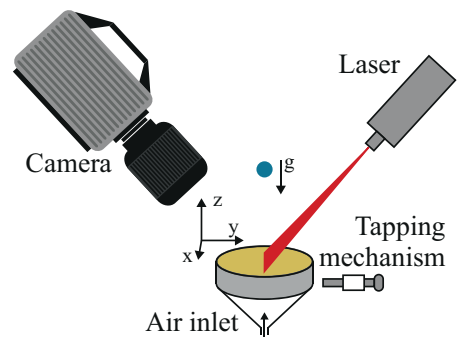

b)

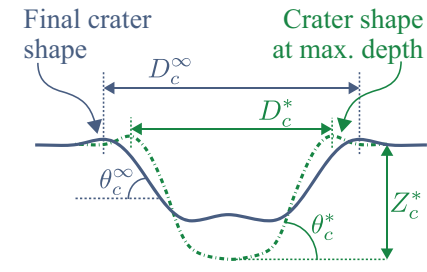

Figure 4.1: $a$ ) Experimental setup of droplet impact on a granular bed. The height profile, $z_{c}(x, y, t)$ is measured with laser sheets and high speed camera. $\left.b\right)$ Relevant length scales of the crater at the moment it reaches its maximum depth and at its final shape.

ular bed and focus a high speed camera (Photron SA-X2) on the target surface, see figure $4.1 \mathrm{a}$. Laser and camera angle are kept between $45^{\circ}$ and $65^{\circ}$ with the substrate. During impact, two parallel laser sheets are used, in combination with a camera frame rate of $10000 \mathrm{fps}$, to acquire a radial height profile $z_{c}(r, t)$ by assuming axisymmetry. When the location of impact is far from the laser lines, the part of $z_{c}(r, t)$ close to the crater center $(r=0 \mathrm{~mm})$ is unresolved. We discard the experiments where the unresolved region is beyond $r=1 \mathrm{~mm}$, and extrapolate the crater profile to $r=0$ with a hyperbola fit otherwise. For more details, see chapter 2 Some minutes after impact, the final crater shape is scanned by carefully translating the substrate horizontally through the laser sheet (accuracy: $16 \mu \mathrm{m} /$ frame), such that a full height profile $z_{c}\left(x, y, t^{\infty}\right)$ can be obtained after analysis, see figure 4.2. When reconstructing the profile $z_{c}\left(x, y, t^{\infty}\right)$, we set the horizontal resolution in the scan direction to the smallest grain size $(70 \mu \mathrm{m}) \sqrt{1}$. In the vertical direction and in the direction along the laser line the minimal resolution is $0.1 \mathrm{~mm}$ per pixel.

From this three-dimensional crater shape $z_{c}\left(x, y, t^{\infty}\right)$ we reconstruct a 2D profile $z_{c}\left(r, t^{\infty}\right)$, by acquiring the crater center and averaging over the azimuthal coordinate. In this procedure, the presence of a liquid residue at the crater base necessitates careful analysis. Hence, to locate the crater center, we restrict ourselves to the crater walls and only consider height contours which are circular. The centers of these contours are averaged and consequently $z_{c}\left(r, t^{\infty}\right)$ is obtained.

\footnotetext{
${ }^{1}$ Note that as the laser is under an angle, we also take into account the horizontal shift when reconstructing the depth $z_{c}\left(x, y, t^{\infty}\right)$.
} 


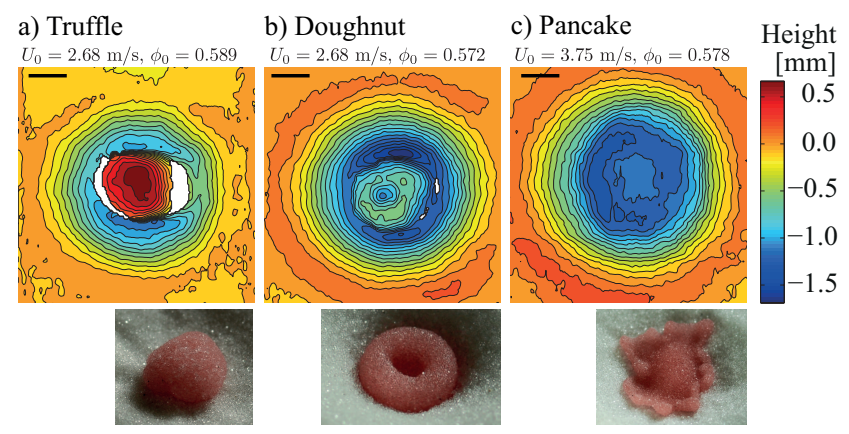

Figure 4.2: Height profiles, $z_{c}\left(x, y, t^{\infty}\right)$, obtained by scanning the crater after impact. Typical liquid-grain residues are denoted as truffle $a$ ), doughnut $b$ ) and pancake $c$ ), and of each an example picture is added, where the liquid was colored red to increase contrast. The bar at the top left of each figure represents $2 \mathrm{~mm}$. White areas in the contour plot lack height information as either laser or camera is blocked due to a sharp height change (as the scan is executed with a translation of the substrate only).

\subsection{Results}

\subsubsection{Final crater profile}

A selection of these averaged final crater profiles $z_{c}\left(r, t^{\infty}\right)$ is illustrated in figure 4.3 . which shows that the crater shape, diameter and depth strongly, and sometimes even non-monotonously, depend on impact velocity $U_{0}$ and packing fraction $\phi_{0}$. One can observe that the final crater depth, measured adjacent to the granular residue, decreases for denser packings, which is plausible as a dense sand bed behaves more solid-like and hence, it is more difficult to deform. The maximum crater depth is reached, however, during impact rather than at its final shape, as contraction of the liquid-grain residue and avalanches tend to make the crater shallower. Therefore, in this chapter we will distinguish two phases: the initial crater opening until its maximum depth is attained and the crater evolution from this moment onward until the final shape has been reached. Besides the above effect on the crater shape, also the shape of the granular residue, i.e., the liquid-grain mixture that remains in the center of the crater after impact, is altered greatly with $U_{0}$ and $\phi_{0}$. A theory, developed in chapter2, explains well the transition observed from the contracted doughnut and truffle residue to the flat pancake shapes (cf. figures 4.2 and 4.3). Here we will concentrate on the behavior of the characteristic length scales of the crater as a function of velocity and packing fraction, and discuss this in the following (sub)sections for both the final and the transient case. 


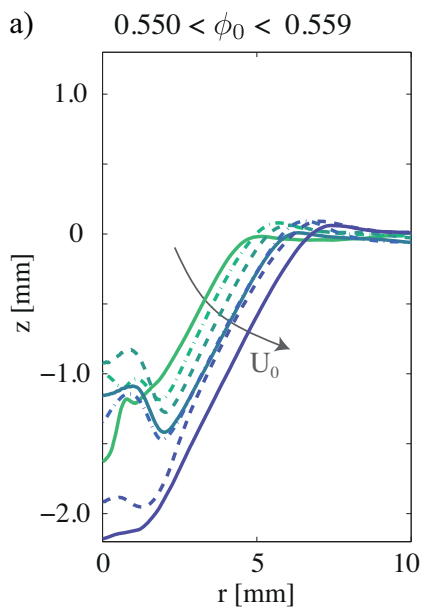

b) $0.570<\phi_{0}<0.574$

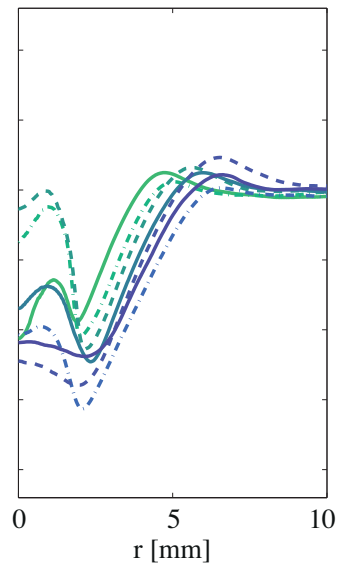

c) $0.589<\phi_{0}<0.595$

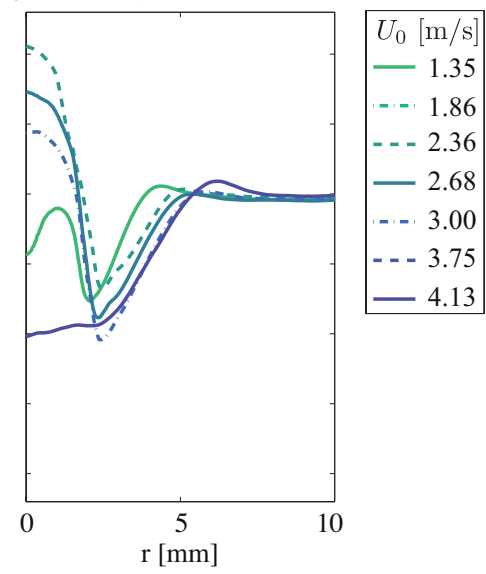

Figure 4.3: Various radial height profiles $z_{c}\left(r, t^{\infty}\right)$ of the final crater are shown for increasing impact velocities and, from left to right, for increasing packing fraction. Note that the center region is occupied by the granular residue, which for all packing fractions changes from a doughnut, through a truffle to a pancake shape for increasing velocity.

\subsubsection{Final crater diameter}

The final crater diameter $D_{c}^{\infty}$, which is defined in this work as the diameter of the final crater shape at the maximum height of the rim (see figure $4.1 \mathrm{~b}$ ), is shown in figure 4.4 in which it is non-dimensionalized with the droplet diameter $D_{0}$. Figure $4.4 a$ displays the data against the kinetic energy of the intruder, $E_{k}=\frac{\pi}{12} \rho_{l} D_{0}^{3} U_{0}^{2}$. Here it is seen that the diameter $D_{c}^{\infty}$ increases with kinetic energy, but scatters greatly with packing fraction. As both intruder and target deform during impact, part of the initial kinetic energy will be transferred into droplet deformation $\left(E_{d}\right)$ and another part into sand deformation energy $\left(E_{s}\right)$. In chapter2 a division of the kinetic energy is proposed related to the stopping force that the drop experiences, $F=E_{k} /\left(D_{0} / 2+Z_{c}^{*}\right)$ with $Z_{c}^{*}$ the maximum depth of the crater (figure $4.1 b$ ). Multiplying the stopping force $F$ with the penetration depth $Z_{c}^{*}$ leads to the following estimate of the energy that is transferred to the granular bed $E_{s}=\frac{Z_{c}^{*}}{D_{0} / 2+Z_{c}^{*}} E_{k}$. When we plot the final crater diameter data $D_{c}^{\infty} / D_{0}$ versus this rescaled energy $E_{s}$ (figure $4.4 b$ ), we obtain a good collapse of the data for various packing fractions, at least for low energies. As indicated in figure $4.4 \mathrm{c}$, the data are consistent with a power-law scaling of $D_{c}^{\infty} / D_{0}$ with $E_{s}^{\alpha}$ with $\alpha \approx 0.16 \pm 0.02$, obtained from a weighted robust fit. The data confirm the hypothesis that a denser 

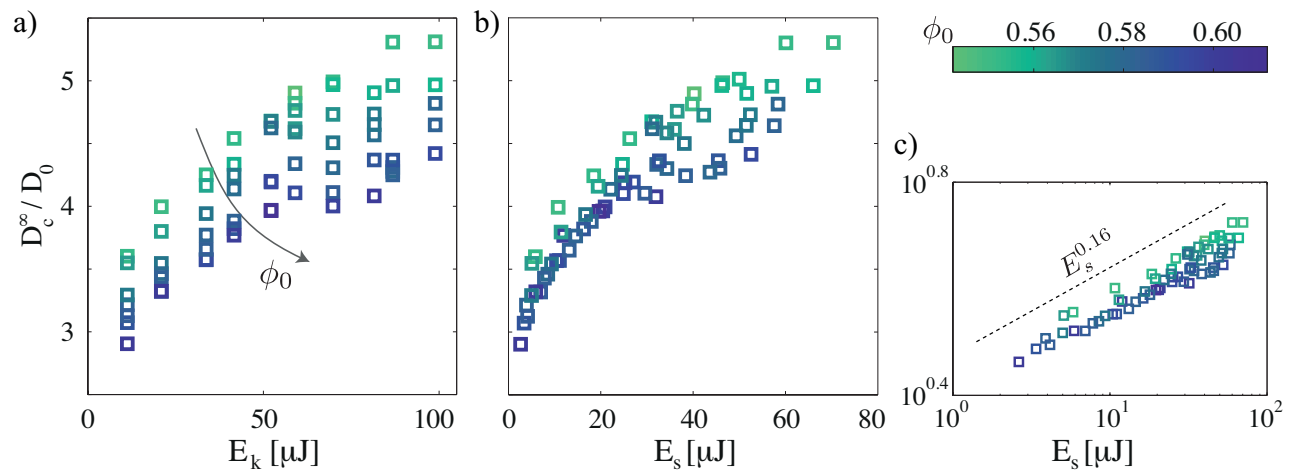

Figure 4.4: Dimensionless final crater diameter is plotted against $a$ ) the kinetic energy $E_{k}$ of the impacting droplet, $b$ ) the estimated amount of kinetic energy transferred to the sand $\left.E_{s}=\left[Z_{c}^{*} /\left(Z_{c}^{*}+D_{0} / 2\right)\right] E_{k} . c\right)$ The same data as in $\left.b\right)$ is shown on a doubly logarithmic scale. The dashed line indicates $D_{c}^{\infty} / D_{0} \propto E_{s}^{0.16}$.

packing leads to a shallower crater due to a smaller energy adsorption.

For the higher energy data in figure $4.4 \mathrm{~b}$, there is however a significant spread with packing fraction. Various mechanisms may be important: after the crater reaches its maximum depth, the crater evolves further, as grains shoot away [20] and avalanches occur before reaching a final state in which the crater diameter is maximal. As a consequence, the final crater shape is formed by complex and subtle processes and therefore, we will focus in the rest of the result section on the evolution of the crater from the moment it reaches its maximum depth onto the final crater shape.

\subsubsection{Crater evolution}

In figure 4.5, the transient crater profile at the moment of reaching the maximum depth, $z_{c}\left(r, t^{*}\right)$ and the final profile $z_{c}\left(r, t^{\infty}\right)$ are compared. One clearly observes how the crater evolves after reaching its largest depth: The crater rim broadens, its depth decreases and the slope of the crater wall diminishes (both for the compact (doughnut or truffle) and flat (pancake) morphology of the residues presented in figures $4.5 a$ and $b$ respectively). Furthermore, it evidences that the crater shape is strongly affected by the packing density, especially for the high impact velocities.

To capture this quantitatively, in figures $4.6 a-c$ we look at the relevant length scales extracted from these $z(r)$ plots, namely the maximum depth $Z_{c}^{*}$, the diameter at the moment of reaching the maximum depth $D_{c}^{*}$ and the final crater di- 

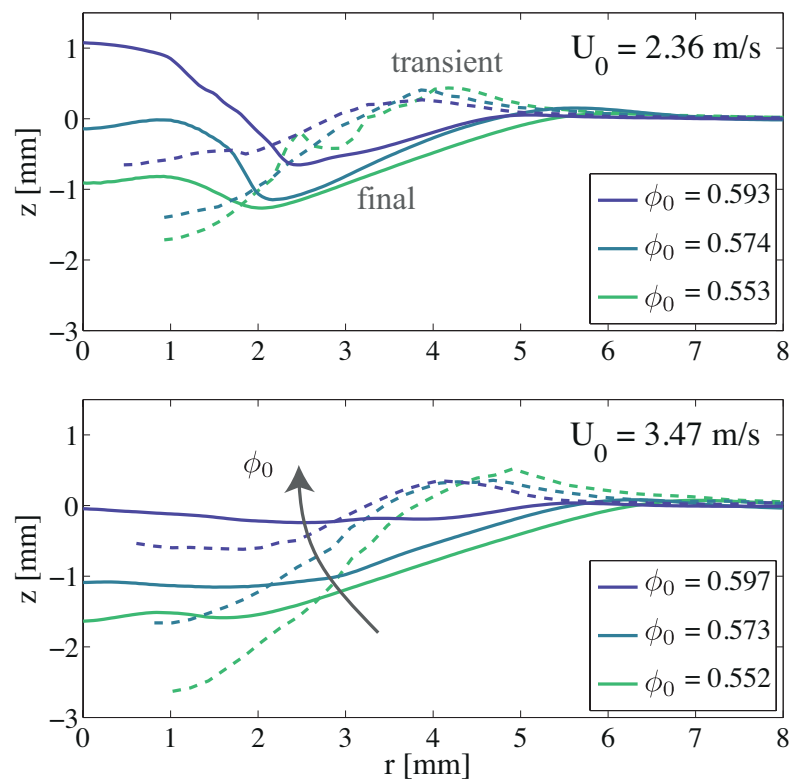

Figure 4.5: Transient and final radial height profiles $z_{c}\left(r, t^{*}\right)$ (dashed lines) and $z_{c}\left(r, t^{\infty}\right)$ (solid lines) for two impact velocities and three different packing fractions. As the droplet impact location is usually at a distance from the laser lines, data for the transient height profile near the center $(r=0)$ is missing.

ameter $D_{c}^{\infty}$, as defined in figure $4.1 b^{2}$. We observe that $Z_{c}^{*}$ and $D_{c}^{\infty}$ noticeably decrease with packing density, but surprisingly, that the transient diameter, $D_{c}^{*}$, hardly varies with $\phi_{0}$. Only for the high velocity cases, one may observe a small dependence. Therefore, the shape and diameter of the crater rim must be affected by the packing fraction mainly in a later stage. This gives the impression that events occurring after the opening of the crater, such as splashing [112, 114] and avalanches [15-117], shape the final crater rim diameter in a profound way. Furthermore, figure 4.6 shows that the transient crater diameter and depth respond differently to packing fraction variations, which we will discuss further in Sec.4.4.3.

What could be the initial driving mechanism for the formation of the crater

\footnotetext{
${ }^{2}$ The crater diameter can also be determined at $z=0$, i.e., the excavation diameter 79,81$]$. For the transient case, we can trustfully do so and it consistently gives a result of approximately $0.8 D_{c}^{*}$. For the final crater shape, the zero base line cannot be determined without ambiguity, and hence, we don't calculate it.
} 


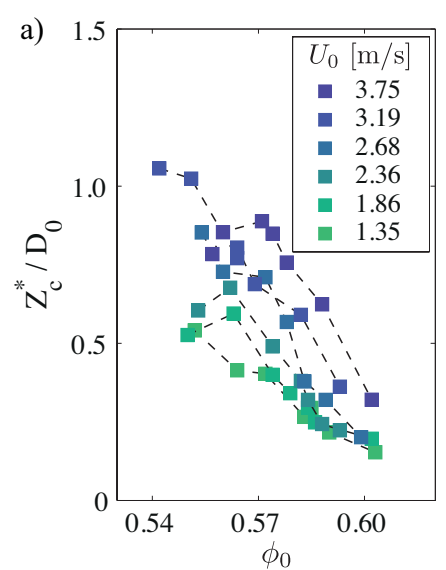

d)

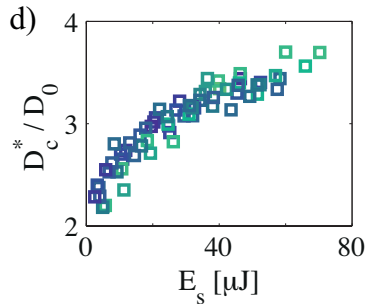

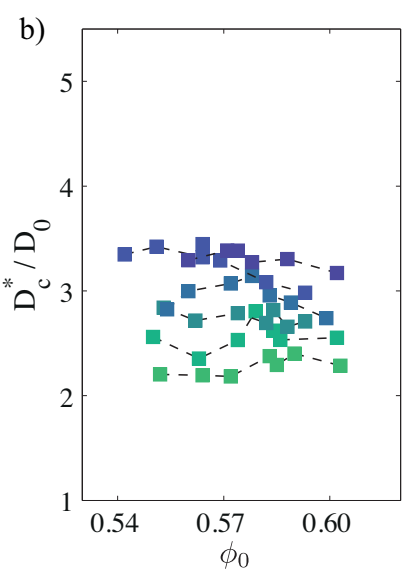
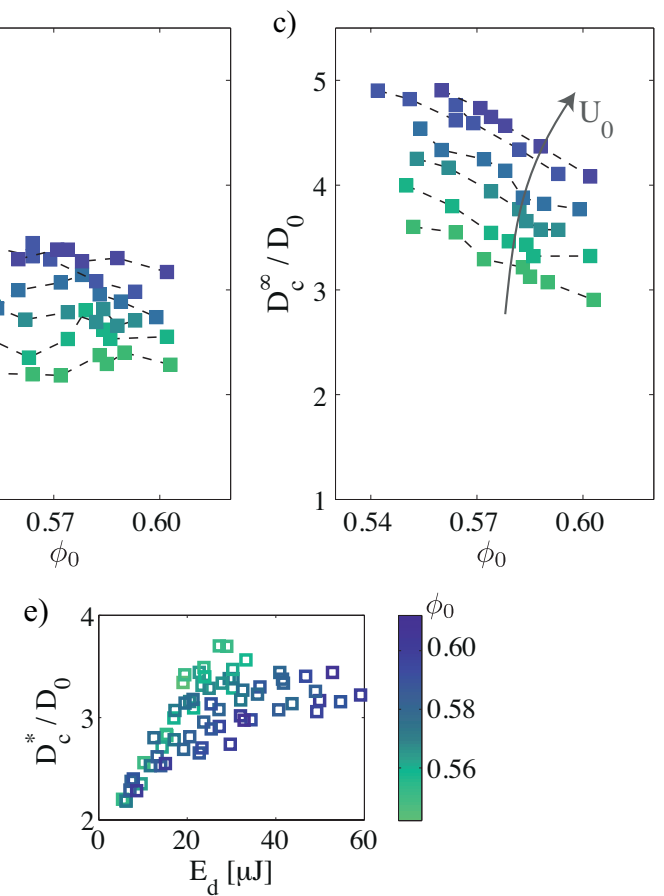

Figure 4.6: $a$ ) -c) Relevant length scales non-dimensionalized with the initial drop diameter $D_{0}$ are plotted against packing fraction with in $a$ ) the maximum depth $Z_{c}^{*}$, in $b$ ) the crater diameter $D_{c}^{*}$ at the moment the maximum crater depth is reached, and in $c$ ) the maximum diameter $D_{c}^{\infty}$ of the final crater. The legend in $a$ ) corresponds to all three subfigures. $d$ ) - $e$ ) The transient diameter $D_{c}^{*} / D_{0}$ is plotted versus the energy $E_{s}$ transferred to the sand $d$ ) and versus the energy $E_{d}$ transferred to the droplet $e$ ). Clearly $E_{s}$ does collapse the data whereas $E_{d}$ does not. For both figures the color bar at the right indicates the packing fraction. 
rim $D_{c}^{*}$ ? It is natural to consider the droplet deformation, which starts right upon impact, and therefore, could be the main cause for pushing grains away. If so, it would be plausible that $D_{c}^{*}$ scales with the kinetic energy imparted on the droplet $E_{d}=E_{k}-E_{s}=D_{0} /\left(D_{0}+2 Z_{c}^{*}\right) E_{k}$ rather than that going into the sand. We find, however, that the data in figures $4.6 d-e$, collapses nicely with $E_{s}$ and not at all with $E_{d} 3$, and hence emphasizes the validity of our approach to divide the initial drop energy into deformation of both the sand bed and the droplet intruder. This result indicates that rather than droplet spreading it is the stress inside the granular bed that is redirected towards the free surface [61, 118, 119] that determines the location of the transient rim. Furthermore, as the $D_{c}^{*}$ data smoothly collapses, the broadening of the final diameter $D_{c}^{\infty}$ versus $E_{s}$ that is observed for high energy in figure $4.4 b$ is most likely due to the post crater formation evolution.

\subsubsection{Crater slope}

The final diameter $D_{c}^{\infty}$ is always larger than the transient diameter $D_{c}^{*}$ (figure 4.6). This implies that the crater broadens and that the slope of the crater walls changes from an initially steep to a flattened-off state, which can also be observed in figure 4.5. We extract the angle of each crater wall averaged around the maximum crater slope, thereby excluding the residue, and plot the result in figure 4.7 as a function of packing fraction. The transient angle lies between $20^{\circ}$ and $55^{\circ}$, scatters with impact velocity and decreases for increasing packing density of the bed. The final angle varies around a value a bit below the angle of repose of $28^{\circ}$ (which we measured using the funnel method), in contrast to the much smaller final crater angle reported in experiments of solid ball impact and the collapse of a cylindrical cavity in granular media [83, 120]. It has been shown from a phenomenological model (of the BCRE-type 4 ) that the final crater slope depends significantly on the initial condition [121, 122]. Therefore, one plausible reason of our comparatively large final angle observations is that the transient crater angle never exceeds $50^{\circ}$, while the collapse of a cavity created by solid ball impact are typically at a higher impact energy which may well cause much larger transient angles. Additionally, the spreading of the droplet may act as a stabilizing factor in the early crater formation, as upon impact a lot of its momentum is converted sideways. In our re-

\footnotetext{
${ }^{3}$ In chapter2 it has been shown that this energy partition collapses the data of both the maximum droplet spreading diameter and the maximum crater depth, when packing fraction is varied. We suspect that the transient crater diameter data will only collapse with $E_{d}$ if the target's response is solid-like where solely the first layer(s) of grains are affected.

${ }^{4}$ These BCRE type of models, named after Bouchaud, Cates, Ravi Prakash and Edwards, separate the granular substrate into a part that is moving down and a static phase.
} 


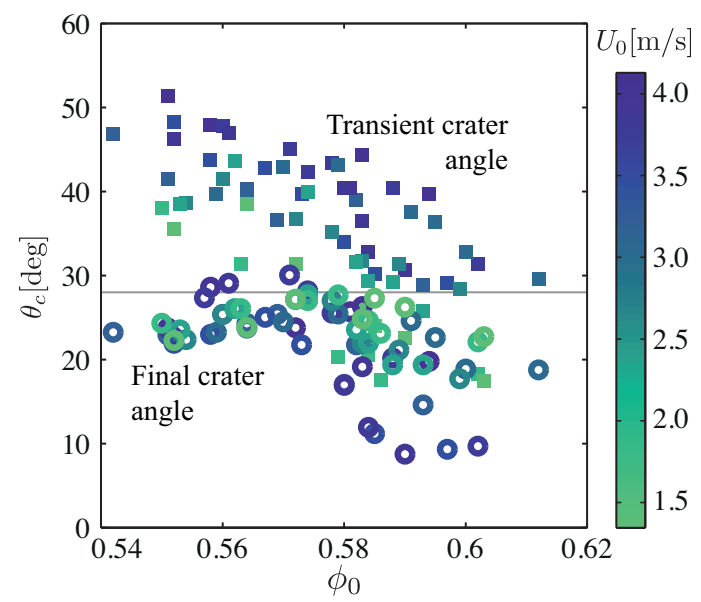

Figure 4.7: Final crater angle $\theta_{c}^{\infty}(\bigcirc)$ and the transient crater angle $\theta_{c}^{*}(\boldsymbol{\square})$ at the moment the maximum crater depth is reached, measured around the maximum crater slope, and plotted versus the packing fraction $\phi_{0}$. The first varies around a slightly smaller value than the angle of repose of $28^{\circ} \pm 2^{\circ}$ indicated by the horizontal solid line.

sults, the variation in the final angles is larger for dense packings $\left(\phi_{0}>0.58\right)$, and the final angles are especially small for the largest impact speeds. We speculate that for those cases, the pancake-shaped residue plays a hard-to-observe-role in producing small final crater angles. In general, the fact that the final angles are close to the angle of repose indicates that avalanching acts as the main mechanism that produces the final crater shape. Combining this information with the observation that the transient depth is greatly affected by packing fraction, but the transient diameter not (as seen from figure 4.6), it implies that avalanches (and maybe splashing) will modify the final crater shape more substantially for looser beds than for the denser ones. This can be seen from the difference between the transient slope and the final one. For example, it is plausible that for an initially deeper crater, more grains will avalanche than for a shallow transient crater (see also the next section). This might be the most important cause for the broadening of the data in figure $4.4 b$.

\subsubsection{Volume displacement}

Lastly, we want to discuss the volume displacement from the moment of reaching the maximum depth to the final crater shape. By comparing the transient and final profiles in figure 4.5 , one can see that volume has moved away from the 
a) $0.551<\phi_{0}<0.553$

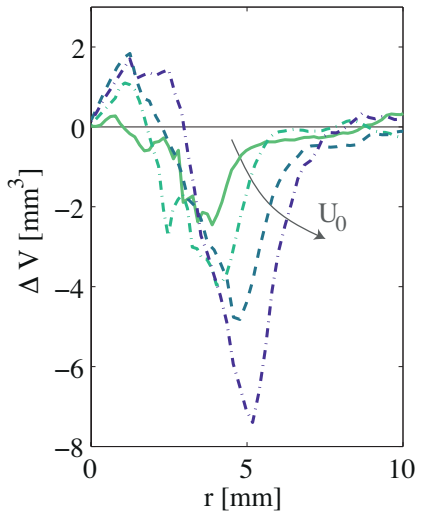

b) $0.560<\phi_{0}<0.564$

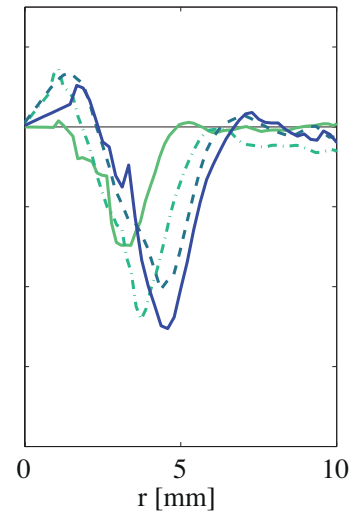

c) $0.590<\phi_{0}<0.594$

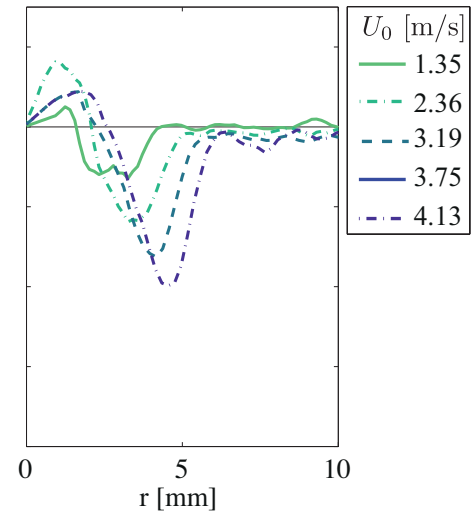

Figure 4.8: Differential volume change $\Delta V$ between the final $z_{c}\left(r, t^{\infty}\right)$ and transient $z_{c}\left(r, t^{*}\right)$ crater profiles are shown per radial distance for increasing impact velocity. It is denoted as: $\Delta V=2 \pi r \Delta r\left[z_{c}\left(r, t^{\infty}\right)-z_{c}\left(r, t^{*}\right)\right]$ with $\Delta r=0.18 \mathrm{~mm}$. The packing fraction is increasing from $a$ ) to $c$ ).

outer crater wall, i.e., near the rim, whereas volume is gained at the center. In figure 4.8 we plot the differential volume between the final and transient shapes, $\Delta V=2 \pi r \Delta r\left[z_{c}\left(r, t^{\infty}\right)-z_{c}\left(r, t^{*}\right)\right]$, as a function of the radius 5 . It shows clearly that the volume loss towards the rim is more significant than the central volume increase, and that the volume change is larger for looser packings, especially for higher impact velocities. The gained central volume in figure $4.8 a$ also increases with impact velocity, which we believe originates from avalanches. We suspect that the packing fraction dependence is mainly caused by the residue, which contracts differently for compact (truffle or doughnut) and flat (pancake) shapes, namely sideways or a bit upwards respectively (see chapter 2).

Around the crater rim, a volume increase should be expected, both through grains that splash away and due to the compression at the center that causes grains to move outwards, towards the free surface. This volume is susceptible to errors (as it is relatively far from the center) and hence, cannot be determined reliably. However, as we observe that the volume loss is greater than the gain, one possible explanation would be that the volume is transferred outwards, to the side of the

\footnotetext{
${ }^{5}$ We choose to plot the radial volume difference and not the total volume, as minor systematic errors may cause a large variation in the total volume. For exammple, for the final crater profile, the zero level has a small systematic uncertainty, which will however contribute significantly to the total crater volume when integrated over the radial coordinate.
} 
crater. It has to be noted that the total displaced crater volume is only expected to become zero for a sand bed at an initially critically packed state, i.e., $\phi_{0}=\phi^{*}$, as shown by [68, 94], as in this case the sand is likely to behave closest to an incompressible fluid.

Regardless the physical reason, we observe that at high impact velocities $U_{0}$, the crater evolution as it is expressed in, e.g., the slope change and volume loss, is much more pronounced and hence we can expect that the crater diameter data gets more dispersed for larger sand energies in figure 4.4 .

\subsection{Discussion}

In addition to reporting and obtaining new insights by data acquisition, we also want to reflect and compare with previous work. We elaborate on two topics, the scaling of the final crater diameter with impact energy and the aspect ratio of the crater, in which case the above presented results may shine new light.

\subsubsection{Maximum crater diameter}

In literature various scalings have been obtained for the maximum crater diameter after drop impact. Some report a scaling with Weber number $\left(\mathrm{We}=\rho_{l} D_{0} U_{0}^{2} / \sigma\right.$ ) [15-17], whereas others determine scalings with the initial kinetic energy of the droplet [18, 21]. Since the impact velocity $U_{0}$ is the most prominently varied control parameter, we may look at the simpler relation of $D_{c}^{\infty}$ with velocity, i.e., $D_{c}^{\infty} \propto U_{0}^{\beta}$, where $\beta$ is found to range from about $0.34[18,21]$ up to 0.50 [15-17]. Also for variation with droplet size, different dependencies are reported. Nefzaoui and Skurtys [18] obtain an experimental fit of $D_{c}^{\infty} \propto E_{l}^{0.18}$ which matches most of their data, where, in the Cheng group, Zhao et al. [21] find a fit of $D_{c} \propto$ $D_{0}^{0.32} E_{k}^{0.17} /\left(\rho_{l} g\right)^{0.17}$, including an additional term containing the droplet diameter and $g$ the gravitational acceleration. In [16, 17], the drop size is simply included in the Weber number.

The influence of the bulk density of the bed, $\rho_{g} \phi_{0}$, is studied by Katsuragi [16], where in order to collapse the data a pre-factor in front of his $\mathrm{We}^{1 / 4}$ dependence is included, which consists of the granular bulk density over the liquid density. Packing fraction variation is considered only theoretically by Holsapple and Schmidt [69], who expect that, for large energy impact of solid intruders on sand or rocks, an increasing compressibility should alter the scaling exponent from what they call energy to momentum scaling.

In this chapter, we have varied impact velocity and packing fraction separately. 
For a given $E_{k}$, a large packing fraction dependence of $D_{c}^{\infty}$ can be observed in figures $4.4 a$ and 4.6 c, i.e., $D_{c}^{\infty}$ decreases with $\phi_{0}$, which is in contradiction to the bulk density dependence suggested by Katsuragi [14]. We find, however, that the data collapses with the part of the kinetic energy going into the sand, $E_{S}$ (cf. figure4.4b), where the stiffness of the sand bed is taken into account. A power-law scaling of $D_{c}^{\infty} / D_{0}$ with $E_{s}^{\alpha}$ with $\alpha \approx 0.16 \pm 0.02$ is obtained. For the nondimensional transient crater diameter a fit with $E_{s}$ gives a power-law exponent of $0.15 \pm 0.02$.

We believe that the variation in reported scaling laws may be related to the fact that in these works, it was not considered that not all initial kinetic energy $E_{k}$ of the impacting drop will be transferred into the sand, but also goes into drop deformation. The partition of $E_{k}$ will depend on the relative stiffness of intruder and target, where the one which is relatively easier to deform, will obtain most of the droplet kinetic energy. Here the extreme cases are the impact of a solid intruder on a granular bed and a deformable drop on a solid target. As we vary the packing density of our bed, this relative deformability is altered, since the stiffness of the bed increases at higher packing fractions. With the inclusion of the (experimentally measured) pre-factor in front of the kinetic energy, i.e., by introducing the portion of the impact energy that is transferred into the sand, $E_{s}=\frac{Z_{c}^{*}}{D_{0} / 2+Z_{c}^{*}} E_{k}$, we effectively account for the varying deformability that originates from changes in the packing fraction (figures $4.4 b$ and $4.6 d$ ). Note that $Z_{c}^{*}$ crucially depends on both kinetic energy and packing fraction, and with that also the just mentioned pre-factor $Z_{c}^{*} /\left(D_{0} / 2+Z_{c}^{*}\right)$. Therefore, the sand energy and initial kinetic energy of the drop do not have to be equivalent (i.e., $D_{c}^{\infty}$ does not scale with the same power with $E_{k}$ as with $E_{s}$ ). All in all, we think that the effect of the relative deformability together with avalanches, constitutes the origin for the variety in power laws that have been observed in previous studies for the maximum crater diameter.

\subsubsection{Excavated mass}

In the dimensional analysis of crater formation by solid intruders, the impact energy is commonly balanced by either plastic deformation or by gravitational excavation [79, 80], i.e., to lift grains against gravity. If the latter is the largest energy sink, and the crater dimensions would scale similarly, the dimensionless maximum diameter scales with the dimensionless energy with a power 1/4 (as also observed in [81, 82]). In our case however, the power differs from 1/4. Furthermore, we find that the excavated mass is not constant when packing fraction is altered: the crater volume decreases more with packing fraction than can be explained from the increase of the bulk density $\left(\rho_{g} \phi_{0}\right)$. More specifically, as can be seen in figure 4.6, the transient crater volume, $\sim D_{c}^{* 2} Z_{c}^{*}$, decreases by about two 


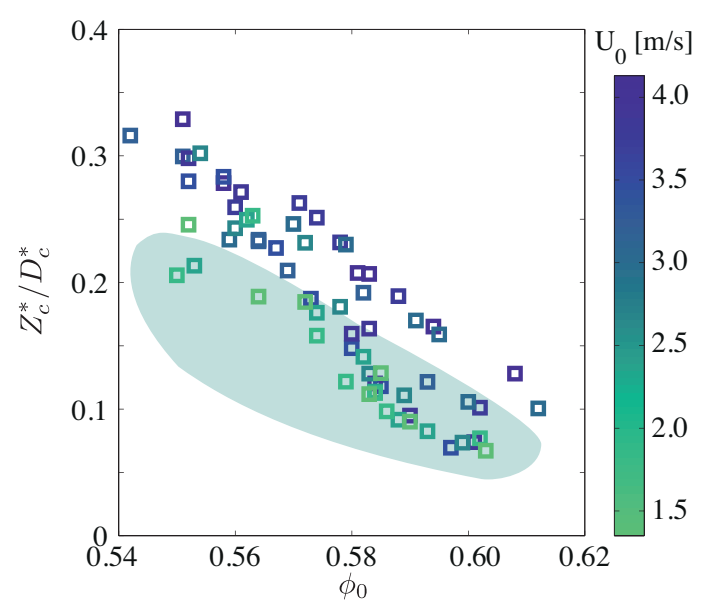

Figure 4.9: Ratio between the maximum crater depth $Z_{c}^{*}$ and the crater diameter $D_{c}^{*}$ at the moment at which the maximum crater depth is reached, is plotted against packing fraction. The light (green) area shows the location of the data cloud for the ratio of $Z_{c}^{*}$ to the final crater diameter $D_{c}^{\infty}$, i.e., $Z_{c}^{*} / D_{c}^{\infty}$.

thirds with an increase of $\phi_{0}$ of only $12 \%$. Therefore, $D_{c}^{\infty}$ will not collapse with the impact energy rescaled by the typical potential energy corresponding to the bulk density, $E_{k} /\left(\rho_{g} \phi_{0} g D_{0}^{4}\right)$. Lastly, we can make a rough estimate of the gravitational potential energy of the final crater shapes, $E_{p} \propto \sum 2 \pi \rho_{g} \phi_{0} g z_{c}^{2}\left(r, t^{\infty}\right) r \Delta r$, looking only at the excavated part (i.e., excluding the rim) and find that $E_{p}$ reaches at most $3 \%$ of the initial impact energy. Moreover, $E_{p}$ decreases with packing fraction. These findings indicate that dissipation processes during the deformation of the granular target are of paramount importance, and since the power-law exponent deviates from $1 / 4$, the energy loss increases with $E_{s}$ in a non-linear manner.

\subsubsection{Crater depth versus diameter}

For planetary and solid intruder impact craters, where the impact energy is usually much larger than for droplet impact, it has often been argued or assumed that the typical crater dimensions, e.g., diameter and depth, scale similarly with impact energy $179,80,123$. There are studies that actually measure the final depth and diameter of the crater. From data of planetary craters, refs. 79, 111 reported a constant depth-to-diameter ratio for so-called simple craters. For smaller impact energies, namely for object impact onto sand, this ratio was only obtained for large grain size $(>180 \mu \mathrm{m})$ [81]. However, de Vet and de Bruyn [83] find that 
depth and radius scale differently with energy. For the first they report an extra dependence on projectile density and for the latter with intruder radius. Uehara et al. [82] investigated the maximum depth (measured below the intruder) and the final diameter and acquired different dependencies on impact energy. For droplet impact on sand, Zhao et al. [21] reported again a constant ratio of depth to diameter of 0.2 , similar to [111], where they compare the depth of the crater at the moment the droplet-grain mixture rebounces (which happens for a subset of the impacts) and the final crater diameter. There they did vary the material properties of the sand, but the packing fraction was fixed to a high value.

In figure 4.9, we plot our result for the ratio of the maximum crater depth $Z_{c}^{*}$ and the diameter at maximum depth $D_{c}^{*}$ and observe that it has a clear dependence on packing fraction and scatters slightly with velocity. When comparing $Z_{c}^{*}$ with the final crater diameter $D_{c}^{\infty}$ as, e.g., was done in [21] (indicated by the green shaded area in figure 4.9), these dependencies do become smaller but do not disappear. The earlier mentioned ratio of 0.2 lies within our data range.

To reconcile our findings with those of Zhao et al. 21], we compare the way in which the ratio has been measured. In [21] the depth is acquired for the cases where a droplet-grain mixture bounces upward. This rebounce occurs at a much later time ( $\sim 40 \mathrm{~ms}$ after the impact) than the moment at which the maximum depth $Z_{c}^{*}$ is reached in our experiments (droplet spreading time scale $\sim 5 \mathrm{~ms}$ ). During this time interval the crater may be modified by e.g., avalanches and mixing of grains into the droplet. Therefore, these two depth measurements may differ in nature. However, as we did not observe droplet rebounce in our experiments, we cannot directly compare these two different methods. To justify the results in figure 4.9, we need to clarify that there are two sources of uncertainties in the measurements of $Z_{c}^{*}$. One stems from the refraction of light through the liquid film which introduces a systematical underestimation of $Z_{c}^{*}$, and the other is introduced by the hyperbola extrapolation used for the unresolved crater centers (cf. figure 4.5. We estimated the uncertainties and find that the tendency shown in figure 4.9 is robust 6 . We want to stress that Zhao et al. [21] obtained the depth only

\footnotetext{
${ }^{6}$ The largest uncertainty introduced by refraction is $\sim 0.14 \mathrm{~mm}$ which is very close to our depth resolution $0.1 \mathrm{~mm}$. This results in a slight underestimation of the maximum depth that is proportional to the thickness of the liquid film at maximum spreading which decreases with packing fraction and impact energy. For a more detailed discussion, we refer to footnote1in chapter2 Note that the reflectance from air to water is negligible, as according to the Fresnel' equations, it is $<10 \%$ and in our experiments the deformed liquid is concave, resulting in an even smaller effect. To estimate the uncertainty of extrapolation, we select a case where the crater center depth is well measured. We fit a hyperbola to the crater profile for $z<0$ and $r>1 \mathrm{~mm}$. The difference between the fitted depth and the measured one is $0.06 \mathrm{~mm}$. Both uncertainties are less than $2 \%$ of $D_{c}^{*}$ and $D_{c}^{\infty}$ and hence, their influence on the aspect ratio is negligible.
} 
for a subset of their experiments, where we acquired data for all impacts that we have studied.

To understand the reason for a non-constant aspect ratio in our data, one may refer to the opening phase of the crater. We see that the transient crater diameter, $D_{c}^{*}$, collapses simply with $E_{s}$, and no packing fraction dependence is observed (figure $4.6 d$ ). For the maximum depth $Z_{c}^{*}$, however, we observed that there is both a (distinguishable) dependence on sand energy and on packing fraction (see figure 2.5 b). This suggests that the crater depth and diameter are determined by different mechanisms, at least for low intruder energies. For high energy or planetary impacts, the material around the impactor might rather get destroyed or molten and hence, the liquid-like material below and beside the impactor would respond similarly leading to the observation of a more constant aspect ratio.

\subsection{Conclusion}

In this chapter, we have shown that in crater formation after the impact of a droplet onto a granular material subtle and complex processes play a role. We compared the transient crater shape, determined at the moment that the crater reaches its maximum depth, and the final crater profile. By including a measure for the relative stiffness of intruder and target, we could approximate the portion of the initial kinetic energy going into sand bed deformation. As can be seen in figures 4.4 and 4.6 $d-e$, both the transient and final crater diameter collapse with this quantity. For the transient crater diameter all packing fraction dependence is captured by using the energy division, however, for the final diameter at the largest sand energies a dependence is still observed. Avalanching is likely to be responsible for this diameter evolution, as it becomes more pronounced for looser beds (figure 4.7). In addition, we surprisingly observed that in the transient phase, the crater may respond differently in the downward and outward direction, as in figures $4.6 a-b$ the depth displays a clear packing fraction dependence which is not seen for the diameter. 



\title{
Impact cratering in sand: solid intruders vs deformable droplets
}

\begin{abstract}
|
How does the impact event of a deformable droplet differ from the one caused by a solid impactor? In this chapter we want to study this question and focus on the effect of intruder deformability on the crater shape. We will show that the crater diameter is larger for droplets than for solid intruders, but that the latter result in deeper craters than for deformable objects. Interestingly, we find for initially dense beds of packing fractions larger than 0.58 , that the resultant excavated crater volume is independent of the tested intruder variation, indicating that the dissipation mechanism in the sand is the same.
\end{abstract}

To be submitted as Rianne de Jong, Song-Chuan Zhao and Devaraj van der Meer, "Impact cratering in sand: solid intruders vs deformable droplets". 


\subsection{Introduction}

Impacts of objects on sand and soils are often observed in Nature. This is not only true on a small scale (e.g., raindrops), but equally on a very large scale with asteroid impacts, the craters of which can be seen on the moon, mars and even our own planet [70, 72]. For planetary craters, it has often been assumed that the intruders are rigid in shape, and accordingly various lab-scale experiments were performed, for which similarities were found between the small craters caused by solid impactors on the laboratory scale and the planetary ones [79-81, 123]. Recently, it was suggested that small scale solid intruders do not quite resemble asteroids, as the latter are expected to fluidize when they hit the target. Pacheco-Vázquez \& Ruiz-Suárez [113] studied impact of granular intruders on sand and found similarities with impact craters on the planetary scale [113, 124]. More specifically, they found that if the yield strength of the impactor is overcome during impact, and hence, it breaks, the resultant crater shapes are similar to craters from asteroid impacts; especially the inner crater shapes qualitatively agree. Moreover, they observed that the craters caused by granular intruders are broader then those of solid intruders. Finally, comparisons were made between liquid drops and large-scale craters [21], where it was found that crater dimensions in both cases follow similar scaling laws.

In all of these works however, the comparison between a solid and a deformable liquid intruder on the laboratory scale is lacking. It is precisely this question that we will address in this chapter, namely to what extent the impacts of a solid and a liquid intruder are different in the first place. A clear distinction is that for liquid intruders impact on sand, the droplet continuously deforms upon impact until a maximum droplet spreading is reached [14-18, 21, 86, 94, 110]. Therefore, as the droplet deforms upon impact, not all of the impact energy is transferred to the sand bed, but goes into droplet deformation as well. In Sec.2.3.2 we proposed a partitioning of the initial kinetic energy into energy going into either drop or sand deformation, that depends on the relative stiffness of intruder and target. Furthermore, for droplet impacts the liquid and grains mix, resulting in various morphologies at the center [14, 17, 86, 94]. Despite the marked difference in intruder response upon impact, are there similarities between the crater shapes observed for the two types of intruders?

In this chapter, we want to answer this question by comparing the impact on sand of a deforming droplet versus a solid impactor of similar size and density as a water droplet. We will use hydrophobic sand to prevent mixing of liquid and grains, such that we focus solely on the effect of the deformability of the intruder. We study these craters by using (high-speed) laser profilometry, and look into typ- 
ical crater measures such as the maximum crater diameter in Sec. 5.3, the maximum depth in Sec.5.4 and the final excavated crater volume in Sec.5.5,

\subsection{Experimental methods}

In our experiments we compare two kinds of intruders: the first are deformable millimeter-size water drops, mixed with food dye to increase contrast (mass fraction $<2 \%$ ) and the second are solid intruders of similar dimension and density as the water droplets. The diameter of the water drops is mostly $2.8 \mathrm{~mm}$ and occasionally $3.5 \mathrm{~mm}$. Three sizes of spherical solid intruders are used, with diameters of 2.01, 3.28 and $4.00 \mathrm{~mm}$, for which the smallest and largest size are made of polypropylene and the intermediate ones out of polyethylene. The solid intruders are painted white to make them opaque, such that we have a proper reflection when we image them. For every size, we paint a batch of particles, as the intruders are small and therefore easily lost during the experiments. The mass of each intruder size is measured by averaging over a group of the solid balls as the paint layer may vary slightly in thickness per intruder, and are for increasing diameter, $3.9 \mathrm{mg}, 17.1 \mathrm{mg}$ and $36.6 \mathrm{mg}$ respectively. This results in densities, $\rho_{i}$, between 900 and $1100 \mathrm{~kg} / \mathrm{m}^{3}$. The solid intruders are pulled into a holder by an underpressure, as the holder is connected to a vacuum pump, and are released when pressure is sufficiently increased by means of the closure of a needle valve. To warrant a smooth release, both holder and intruder are regularly cleaned with ethanol to prevent sticking. In addition, the holder and target are grounded to avoid build up of static electricity. The liquid drops are released from a needle when gravity overcomes surface tension. The impact velocity, $U_{0}$, is varied in a range of $1-5.5 \mathrm{~m} / \mathrm{s}$ by changing the release height and is for every droplet measured just before impact. For the solid intruders the impact velocity is conveniently computed from a calibrated height-velocity profile.

The target consists of a bed of grains. The grains are white, silane coated (i.e., hydrophobic), soda-lime beads (specific density $\rho_{g}=2.5 \times 10^{3} \mathrm{~kg} / \mathrm{m}^{3}$ ) of two batches, of which the mean of the size distributions are $d_{g}=114$ and $200 \mu \mathrm{m}$ respectively. The packing fraction of the bed is varied between $0.55-0.62$. In this first part we focus on a packing fraction range of 0.58 to 0.60 and on the batch consisting of larger grains $(200 \mu \mathrm{m})$ only. The beads are dried in an oven around a temperature of $105^{\circ} \mathrm{C}$ for at least half an hour.

We capture the deformation of the bed surface upon impact with an in-house built high-speed laser profilometer, from which we acquire a dynamic crater profile, $z_{c}(r, t)$, by assuming axisymmetry. With the same laser and camera, and help 
a)

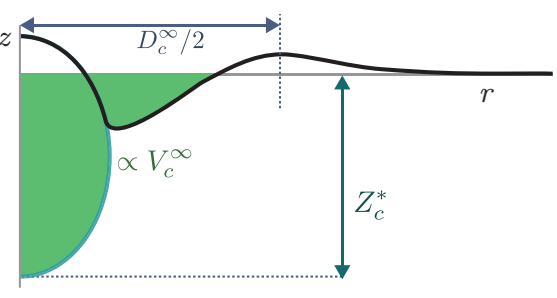

b)

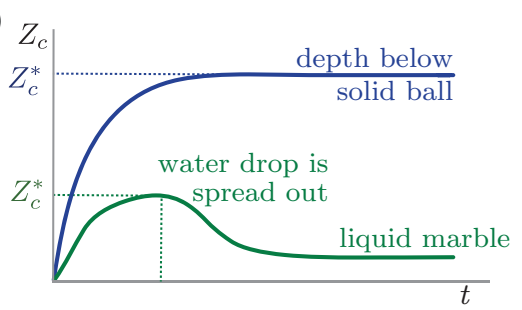

Figure 5.1: $a$ ) The relevant length scales of the crater and the excavated crater volume $V_{c}^{\infty}$ determined from the final crater shape $z_{c}\left(r, t^{\infty}\right)$. b) Time evolution of the center crater depth (at $r=0$ ) for a solid intruder (top line) and water drop on hydrophobic grains (bottom line). For the latter, the maximum depth is only perceivable during impact, as the droplet contracts after the maximum spreading has reached. The maximum depth of a solid intruder will be constant as soon as the object comes to rest.

of a translation stage, the final crater shape $z_{c}\left(x, y, t^{\infty}\right)$ is obtained in 3D. By assuming axisymmetry, we obtain a radial $z_{c}\left(r, t^{\infty}\right)$ profile, from which we acquire the crater dimensions. See chapters 2 and 4 for more details on this method.

\subsection{Maximum crater diameter}

The diameter of the final crater shape, $D_{c}^{\infty}$, obtained here at the top of the rim of the crater, is a typical measure for the target deformation after impact (see figure $5.1 a$ ). As the impact energy that goes into sand deformation is smaller for deformable intruders (see previous chapters), intuition would suggest that for the same impact speed the resulting crater diameter should be smaller for the droplet than for the solid intruder impacts. As the size of solid and liquid intruders varies, we can compare them by non-dimensionalizing the final diameter with the initial intruder diameter $D_{0}$ and the kinetic energy just upon impact $E_{k}=(\pi / 12) \rho_{i} D_{0}^{3} U_{0}^{2}$ can be rescaled with the potential gravitational energy $E_{g}=(\pi / 6) \rho_{s} \phi_{0} g D_{0}^{4}$, corresponding to a cavity with dimensions $D_{0}$ dug into the sand 1 . In figure $5.2 a$, one can see that, in striking contrast to our intuition, the crater diameter is larger when caused by a deformable intruder than by a solid one. This effect is even more pronounced when we take into account that for drop impact, the droplet spreading consumes part of the initial impact energy (figure $5.2 \mathrm{c}$ ). Here, the sand defor-

\footnotetext{
${ }^{1}$ The dimensionless energy $E_{k} / E_{g}$ can also be written as a density ratio times the Froude number, i.e., $\frac{1}{2} \frac{\rho_{i}}{\rho_{s} \phi_{0}} \frac{U_{0}^{2}}{g D_{0}}$.
} 

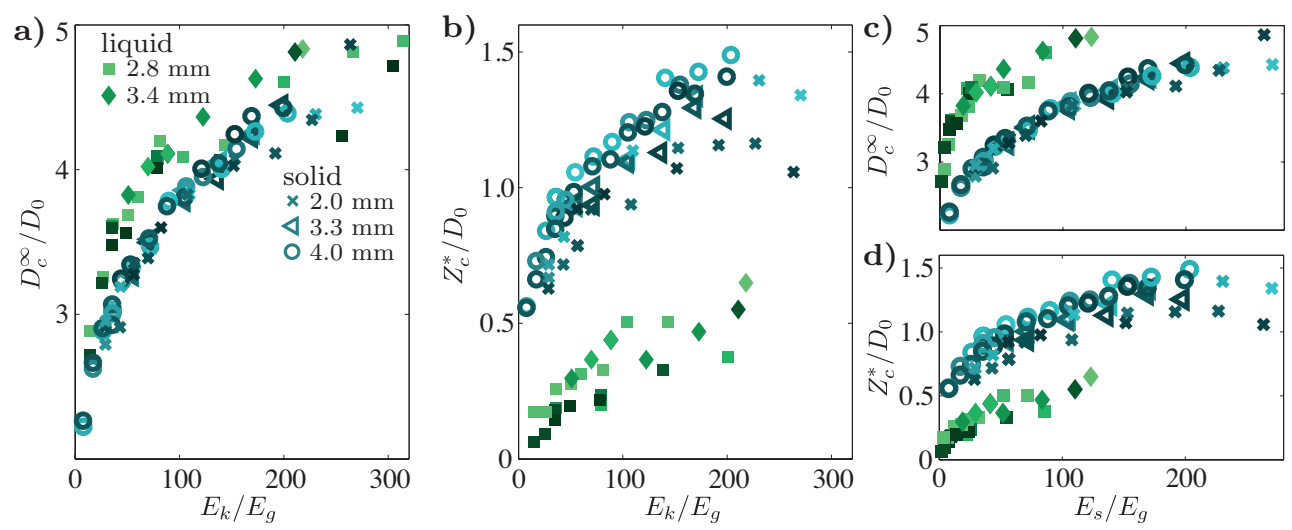

Figure 5.2: $a$ ),b) The maximum dimensionless crater diameter $D_{c}^{\infty} a$ ) and depth $Z_{c}^{*} b$ ) are plotted against the non-dimensional kinetic energy $E_{k} / E_{g}$ for deformable water droplets (filled green $\square, \diamond$ symbols) and solid balls (blue $\times, \triangleleft$, ० symbols ). The packing fraction is limited to the range $\phi_{0}=0.58-0.60$, where the color of the symbols darkens with increasing $\phi_{0}$. Only data for grains of mean diameter $d_{g}=200 \mu \mathrm{m}$ are shown. $c$ ), $d$ ) The same data sets are plotted as in $a, b$ ), but for the droplet data only the part of the kinetic energy going into the deformation of the sand $E_{s}$ is taken into account (and $E_{s}$ is simply equal to $E_{k}$ for solid objects). See the main text for the definitions of $E_{s}, E_{k}$, and $E_{g}$.

mation energy $E_{s}$ is given by $E_{s}=Z_{c}^{*} /\left(\frac{1}{2} D_{0}+Z_{c}^{*}\right) E_{k}$ for the droplets, where $Z_{c}^{*}$ is the maximum crater depth discussed in greater detail in the next section, and $E_{s}=E_{k}$ for the solid intruder data. So, our simple hypothesis that a crater caused by solid intruders is larger in all directions than the one formed by a droplet does not hold. The opposite is true: the final crater is narrower for solid intruders than for droplets2.

How does droplet spreading influence the crater diameter to such an extent that it becomes larger than that of a solid impactor? Upon impact, the droplet spreads outwards until a maximum spreading diameter is reached (chapter 3). An intuitive approach used in [14, 17, 72] and implicitly in [21], relates the crater diameter directly to the maximum droplet spreading diameter. We however found no support for this reasoning, but showed in chapter 4 that the crater diameter is already developing independently of drop deformation at a quite early stage of the impact. More specifically, we found that the transient crater diameter (figure 4.6, measured at the moment the maximum crater depth is reached, and the

\footnotetext{
${ }^{2}$ Note that the crater diameter data collapses irrespective of whether it is plotted against $E_{k} / E_{g}$ or $E_{S} / E_{g}$, which is due to the fact that, other than in chapters 3 and 4 the data is restricted to a narrow range of packing fractions.
} 
final crater diameter (figure 4.4) collapses with the sand deformation energy $E_{s}$ and definitely not with the droplet deformation energy $E_{d}=E_{k}-E_{s}$ for variation in packing fraction 3 . In addition, we observed that droplet spreading is greatly reduced when mixing between liquid and grains becomes important, i.e., when wettability of the grains and their size are increased (see chapter 3). If we compare the final crater diameter and maximum droplet spreading diameter for the same set of experiments, we find that they collapse with different parameters, indicating that the final drop and crater diameter are not directly related to one another (when taken into account the amount of energy going into each). See appendix 5.A for further details.

Despite the fact that droplet spreading cannot explain the increase of the crater diameter for droplet impact, it is a very intrinsic feature of a liquid drop that it will deform under forcing. It can redirect the downward momentum partly into horizontal momentum: while it penetrates into the bed, it deforms sideways and can push grains radially outwards 4 . We can understand this better when we look at the difference between solid and liquid intruders impacting onto a liquid bath. A solid intruder will, initially, continue with a velocity close to the impact velocity $U_{0} 5$ A droplet will move into the bath with a much smaller velocity of maximally $U_{0} / 2[125] 6$, simply because both intruder and target are deformable. Seen from a potential flow perspective the streamlines move first vertically inward towards the stagnation point and then bend horizontally outward. For our experiments this means that for solid intruders the forcing due to its inertia is simply directed downwards, but for the deformable drops, this inertial forcing is directed both downward and sideways already right after impact. A large difference between a liquid pool and a granular bed as a target is that for the latter the grains close to the surface can move out freely as soon as they are set into motion and are not slowed down by surface tension. Hence, the broader crater diameter for drop impact does not have to relate to the droplet spreading (which can be quickly slowed down if mixing is important), but connects to the early dynamics that occurs directly after impact.

\footnotetext{
${ }^{3}$ This energy $E_{d}$ is used in chapter[2] to obtain a modified Weber number that collapses the maximum droplet spreading.

${ }^{4}$ This concept of vertical-to-horizontal momentum transfer was also postulated by PachecoVázquez \& Ruiz-Suárez 113] for their experiments of granular ball impact onto a granular substrate.

${ }^{5}$ Upon impact, the liquid will be set into motion by the intruder, giving rise to an added mass force that will decelerate the intruder. By assuming a similar density for intruder and target, the velocity of the intruder and the virtual added mass can be approximated as $2 / 3 U_{0}$.

${ }^{6} \mathrm{~A}$ stagnation velocity of $U_{0} / 2$ is the limiting case for a liquid jet impacting with a velocity $U_{0}$ on a pool. For a single drop it will be smaller and for a much shorter time duration of order $t=$ $\left(D_{0} / 2+Z_{c}^{*}\right) / U_{0}$. 125]
} 


\subsection{Maximum crater depth}

As the droplet deforms and not all the inertial forcing will be directed downward, we can presume that the penetration depth of a drop will be smaller then that of a solid intruder. For the droplet experiments, the maximum crater depth, $Z_{c}^{*}$, is observed during impact (figure 5.1). After the droplet has spread out upon impact, it will contract under surface tensional force and becomes a liquid marble: grains are collected at the interface of the drop, decreasing the center crater depth. We therefore obtain $Z_{c}^{*}$ from the dynamic $z_{c}(r, t)$ profiles. It is easier to acquire for the solid balls, as the solid intruders get rarely buried in our set of experiments. The crater depth will remain unaltered as soon as the impactor comes to rest, and $Z_{c}^{*}$ can simply be found by subtracting the intruder diameter from the center part of the $z_{c}\left(r, t^{\infty}\right)$ profiles. In figure $5.2 b$, we see that for the same impact energy the solid intruder craters become much deeper than for the ones created by the impact of a droplet and this difference persists when we take into account that for droplets not all kinetic energy goes into deformation of the sand by plotting the same data versus $E_{s} / E_{g}$ rather than $E_{k} / E_{g}$ (figure $5.2 d$ ). So we observe that the crater formed by drop impact is broader and considerable shallower compared to the solid intruder crater, consistent with the conjecture that momentum is transferred both downward and sideways for deformable intruder impact.

\subsection{Excavated volume}

Knowing that exchanging a solid impactor for a deformable one simultaneously increases the crater diameter and decreases its depth makes one wonder what may happen with the total excavated volume. We quantify the crater volume $V_{c}^{\infty}$ as the void that is created into the granular bed after impact, i.e., by computing all the granular material that has moved away from below the original surface of the bed before the impact. More quantitatively, we calculate it as $V_{c}^{\infty}=$ $\sum\left(2 \pi z_{c}\left(r, t^{\infty}\right) r \Delta r\right)-V_{i}$, with $z_{c}\left(r, t^{\infty}\right)$ the radial height profile of the final crater, and $V_{i}$ the intruder volume? We only consider the volume until $z=0$ is reached (nearby the crater rim), see figure $5.1 a$ for a sketch. In figure $5.3 a$, the excavated crater volume, normalized with the volume of the intruder $V_{i}$, is plotted against the dimensionless impact energy. The crater volume for the solid intruders impacts is noticeably larger for all impact energies than for the droplet intruder cases. If we plot, however, the volume against the non-dimensional sand deformation energy

\footnotetext{
${ }^{7}$ For the droplet impacts, we assume that the liquid volume that may have splashed away is not significantly affecting this calculation.
} 

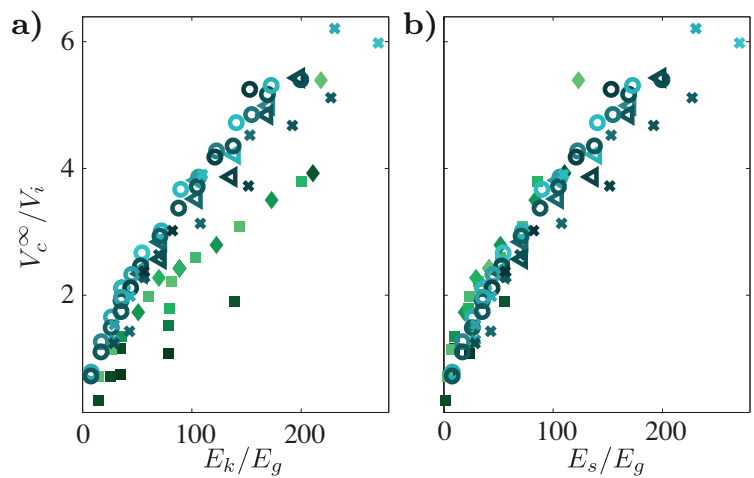

Figure 5.3: The final crater volume $V_{c}^{\infty}$ rescaled with the intruder volume $V_{i}$, is plotted against $a$ ) the non-dimensional kinetic energy $E_{k} / E_{g}$ and $b$ ) the non-dimensionalized portion of the kinetic energy going into sand deformation $E_{s} / E_{g}$ for deformable water droplets (filled green $\square, \diamond$ symbols) and solid balls (blue $\times, \triangleleft$, ० symbols). The same data set, colors and symbols as in figure 5.2 are used.

$E_{s} / E_{g}$, we see the data nicely collapses onto a single curve. This result shows that despite the fact the horizontal and vertical crater dimensions differ significantly, the total energy going into crater formation gives rise to the same excavated volume for a solid and a deformable intruder. Strikingly, it suggests that for this set of experiments, the dissipation processes within the sand yield the same result for the dissipated energy during the impact of the two types of intruders, even if the details of the crater formation process are different. This is even more evident when it is realized that the potential energy stored in the displaced sand volume is found to be less than $5 \%$ of the impact energy, i.e., the crater formation process is heavily dominated by dissipation?

\subsubsection{Packing fraction dependence}

Until so far, we kept the packing fraction range limited to $\phi_{0}=0.58-0.60$. However, for drop impact on sand, we found that the crater shapes and the energy transferred to the sand greatly vary with packing density (see chapter 4). Also for solid intruders, the crater shapes are altered with packing fraction [68], where it was found that the total displaced volume of sand (including the rim) goes from

\footnotetext{
${ }^{8}$ As a matter of fact, also the percentage of momentum going into splashing has been investigated in 20] by tracking splashing grains after drop impact on sand, and it was found that the splash momentum was only about $4 \%$ of the initial droplet momentum before impact.
} 

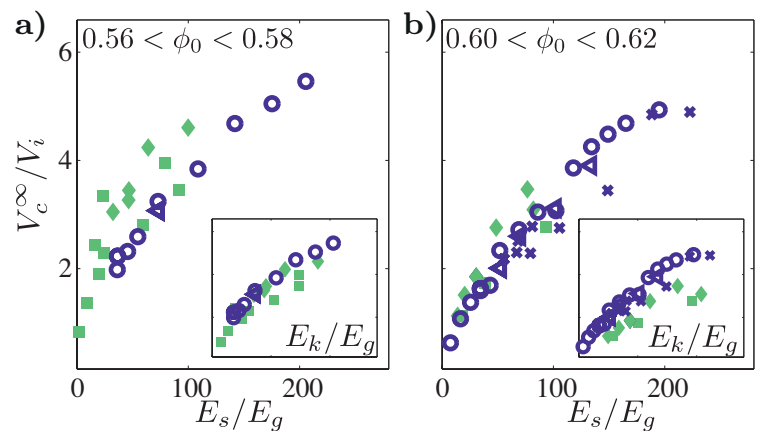

Figure 5.4: The final crater volume, normalized with the intruder volume $V_{i}$, is plotted against the non-dimensional kinetic energy $E_{s} / E_{g}$ for $a$ ) low packing fraction, $0.56<\phi_{0}<$ 0.58 and $b$ ) high ones, $0.60<\phi_{0}<0.62$. The insets display the same data plotted for $E_{k} / E_{g}$ (axis are the same). The same symbols as in figure 5.2 are used, with the droplet data again filled light green and the solid intruder data with dark purple symbols.

a compressed state for loose beds (i.e., the total displaced sand volume is smaller than the crater volume), crosses zero at a critical packing fraction $\left(\phi^{*} \approx 0.59\right)$, to a dilated state for dense beds (where the displaced sand volume is larger than the crater).

In figure 5.4 all excavated volume data for packing fraction ranges from $\phi_{0}=$ $0.56-0.58$ and $\phi_{0}=0.60-0.62$ is displayed and we observe that the data for solid and deformable intruders collapses with $E_{s} / E_{g}$ for the densest packed beds as well. For the loose beds of $0.56<\phi_{0}<0.58$, however, the droplet data lies above the crater volumes formed by solid intruders 9 . These findings are consistent for grains of $d_{g}=114 \mu \mathrm{m}$, see appendix 5.B for a figure and details. Remarkably, for these low packing densities, the data seems to collapse better with the dimensionless impact energy $E_{k} / E_{g}$ (see inset in figure 5.4 a and figure 5.6) rather than $E_{s} / E_{g}$. The energy consumed by drop deformation $E_{d}$ varies between $30 \%-80 \%$ of the impact kinetic energy in this set of experiments and it is therefore a highly unexpected result. We postulate that the apparent collapse of the data with $E_{k} / E_{g}$ is a coincidence. In summary, we observe that the excavated volume $V_{c}^{\infty} / V_{i}$ collapses with the non-dimensional sand deformation energy $E_{s} / E_{g}$ for beds with a packing fraction of $\phi_{0}>0.58$, For loose beds of $\phi_{0}<0.58$, droplet impacts result in larger crater volumes than the ones created by solid intruders.

What could be the reason for this apparent packing fraction dependence? The

\footnotetext{
${ }^{9}$ We want to stress that for the whole packing fraction range, the crater diameter and depth display similar trends as in figure5.2 i.e., the crater shapes differ always for droplet and solid intruders.
} 
answer may lie in the flow and force response of a sand bed that changes significantly around a critical packing fraction $\phi^{*}[66-68,88,90,126]$. Above $\phi^{*}$ the packing will dilate under forcing [127], but for $\phi_{0}<\phi^{*}$ the substrate will get compressed to $\phi^{*}$. For the grains used in these experiments, $\phi^{*} \approx 0.58-0.59$, so the earlier mentioned change around $\phi_{0} \approx 0.58$ from a collapse of all excavated volume data (dense beds) to a regime with a difference in the excavated crater volume for the two types of intruders (loose beds) may be interpreted in the light of the dilatancy onset. For the loose beds, we could imagine that the substrate will first get relatively easily compressed to $\phi^{*}$ upon impact, after which the majority of the dissipation between the grains will occur. This may set the difference between the crater volume of the solid and liquid impactor: the droplet can transfer its energy to the bed more effectively as it can move or deform the bed there where it experiences the lowest resistance, and thus the droplet will be a more effective "compressor", resulting in larger crater volume for the same sand deformation energy $E_{s}$. Further work is necessary to see if this hypothesis holds, e.g., by impact of slightly deformable solid intruders, (such as hydrogels) of varying stiffness onto beds of varying packing density.

\subsection{Discussion}

We directly compared craters formed by the impact of solid intruders and deformable droplets on sand. We found that the crater length scales differ for all cases we studied: solid intruder impact results in deeper but shallower craters compared to the impact of deformable impactors. As explanation we proposed that for droplets the inertial forcing is directed downwards and sideways, but for solid intruders, the forcing will simply be directed downward. This concept of vertical-to-horizontal momentum transfer was also postulated by PachecoVázquez \& Ruiz-Suárez [113] for their experiments of consolidated granular ball impact onto a granular substrate. They observe that, as soon as their granular intruder breaks into pieces, i.e., its yield strength is overcome, the final crater becomes broader and shallower than for solid intruders of the same mass. Where they find that the difference between the crater diameter of the granular intruder and the solid one is constant and independent of impact energy, in our case, the diameters of the solid and the deformable intruder impacts follow different powerlaw scalings. The exponent $\alpha$ for a fit to the functional form $D_{c}^{\infty} / D_{0} \propto\left(E_{s} / E_{g}\right)^{\alpha}$ is for the water drops $\alpha \approx 0.16$ and for the solid intruders $\alpha \approx 0.21$. In addition, in [113] the depth of the crater formed by a granular object goes towards a relatively constant value as soon as the intruder falls apart, whereas for our drops the crater 


\subsection{DISCUSSION}

depth increases with impact energy, see figure $5.2 b$.

Subsequently, we studied the resultant excavated crater volume and found, surprisingly, that for dense beds of $\phi_{0}>0.58$, the volume is independent of the chosen intruder type when taking into account that for droplets, part of the initial energy goes into drop deformation. Since these type of impacts are heavily dominated by dissipation, this indicates that for these dense beds the dissipation processes within the sand bed generate the same amount of dissipated energy during impact of a solid object or a deformable drop. We speculate that the dependence on packing fraction for this observation is related to the onset to dilatancy. Further research is needed to validate this idea. 


\section{A Final crater diameter vs maximum droplet spreading}

In this appendix we compare the maximum droplet spreading diameter $D_{d}^{*}$ with the final crater diameter $D_{c}^{\infty}$ that is reached later in time. We include in the comparison not only the hydrophobic grains of 114 and $200 \mu \mathrm{m}$ that were used in the main text, but also hydrophilic ceramic beads of three sizes: 98,167 and $257 \mu \mathrm{m}$ with two types of wettabilities. See for more details, chapter 3, table 3.1 on page 29. In that chapter, we found that the maximum droplet spreading $D_{d}^{*}$ is greatly reduced when mixing between liquid and grains is important. For simplicity, we replot this data in figure 5.5 $\mathrm{b}$, where on the horizontal axis the modified Weber number $\mathrm{We}^{\dagger}$ is plotted, which is a measure of the deformability of the droplet, and could be written as $\mathrm{We}^{\dagger} \propto E_{d} / E_{g}\left(\rho_{g} \phi_{0} g D_{0}^{2}\right) / \sigma$ (see for more details, Sec. 2.4). In this figure one can see that the diameter decreases for increasing bulk wettability (i.e., the wettability and/or size of the grains). Note that in chapter 3 we developed a theory including the bulk wettability and found a collapse of the data. If the crater diameter is related to the maximum droplet spreading, as postulated by [14, 17, 72], we expect that when we plot these two measures against each other, they collapse onto a single curve (figure $5.5 \mathrm{c}$ ). However, the data of $D_{c}^{\infty} / D_{0}$ versus $D_{d}^{*} / D_{0}$ scatters greatly. In the figure, we indicated the changes that occur with packing fraction and impact energy: both diameters increase with increasing impact energy and when the packing fraction is increased, $D_{d}^{*}$ increases and $D_{c}^{\infty}$ decreases. Furthermore, if we plot the non-dimensional final crater diameter against $E_{s} / E_{g}$ (figure 5.5a), a simple collapse is observed, with no dependence on the bulk wettability. All in all, this shows that the hypothesis that maximum droplet spreading and crater diameter are related, does not hold.

\section{B Excavated crater volume for small grains}

In Sec. 5.5, the excavated crater volume $V_{c}^{\infty}$ for impacts on $200 \mu$ m hydrophobic grains was discussed. Here, we show $V_{c}^{\infty}$ for the same type of hydrophobic grains, but with an average diameter of $d_{g}=114 \mu \mathrm{m}$, see figure 5.6. Similar to the results of the larger grain size, we observe that the crater volume data of droplet and solid intruder collapses with $E_{s} / E_{g}$ for $\phi_{0}>0.58$ (figure $[5.6 c)$ ). For loose beds of $\phi_{0}<0.56$ the data is disperse (figure 5.6 $a$ ) and seems to collapses better with $E_{k} / E_{g}$ (inset), which is an unexpected result (see main text). For the packing fractions between $0.56<\phi_{0}<0.58$, it is indefinite. The excavated volume created by impacts on these smaller grains $\left(d_{g}=114 \mu \mathrm{m}\right)$ result in slightly smaller crater volumes $V_{c}^{\infty} / V_{i}$ then the ones observed on beds with a mean diameter of $d_{g}=200 \mu \mathrm{m}$. 


\section{B. EXCAVATED CRATER VOLUME FOR SMALL GRAINS}
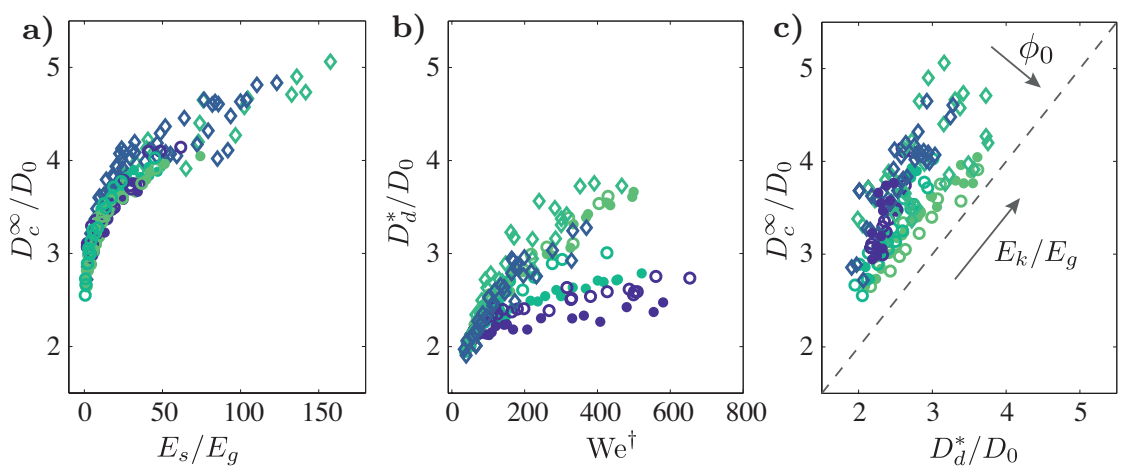

$$
\begin{aligned}
& \text { water on } \\
& \text { ceramic } \\
& \text { - } 98 \mu \mathrm{m} \\
& \text { - } 167 \mu \mathrm{m} \\
& \text { - } 257 \mu \mathrm{m} \\
& \text { water on } \\
& \text { clean-ceramic } \\
& \text { - } 98 \mu \mathrm{m} \\
& \text { - } 167 \mu \mathrm{m} \\
& \text { - } 257 \mu \mathrm{m} \\
& \text { water on } \\
& \text { hydrophobic } \\
& \text { soda-lime } \\
& \diamond 114 \mu \mathrm{m} \\
& \diamond 200 \mu \mathrm{m}
\end{aligned}
$$

Figure 5.5: $a$ ) The maximum crater diameter $D_{c}^{\infty}$ normalized with $D_{0}$ collapses against the non-dimensional $E_{s} / E_{g}$ for water droplet impact on various substrates. $b$ ) The maximum droplet spreading is plotted against a modified Weber number $\mathrm{We}^{\dagger}$ for the same data sets. c) The dimensionless final crater diameter $D_{c}^{\infty} / D_{0}$ is plotted against the dimensionless maximum droplet spreading diameter $D_{d}^{*} / D_{0}$. The arrows indicate the increase in packing fraction $\phi_{0}$ and the dimensionless impact energy $E_{k} / E_{g}$.
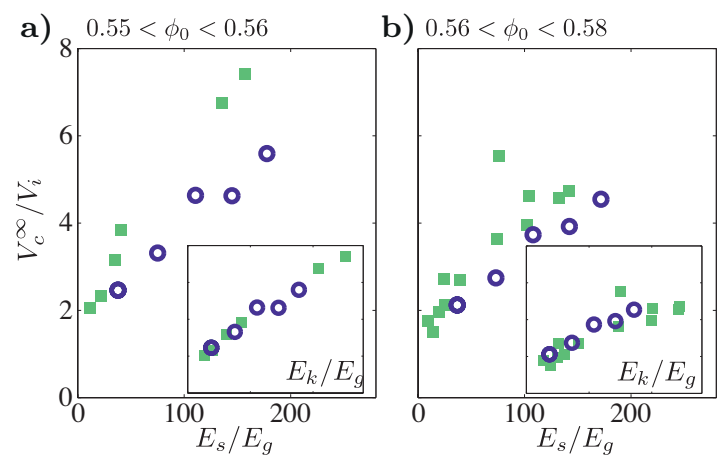

c) $0.58<\phi_{0}<0.60$

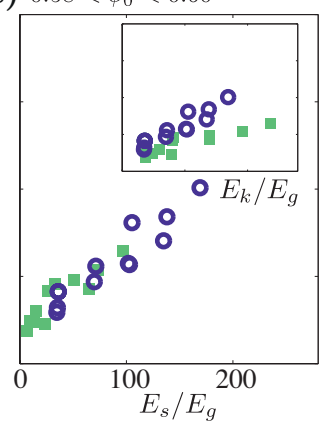

Figure 5.6: The final crater volume $V_{c}^{\infty}$, normalized with the intruder volume $V_{i}$, is plotted against the non-dimensional kinetic energy $E_{S} / E_{g}$ for $a$ ) low packing fractions, $0.55<\phi_{0}<$ $0.56, b) 0.56<\phi_{0}<0.58$ and $c$ ) the same range as in figure 5.3. $0.58<\phi_{0}<0.60$. The insets display the same data plotted for $E_{k} / E_{g}$ (axis are the same). The same symbols as in figure 5.2 are used, with the droplet data again filled light green and the solid intruder data with open dark purple symbols. Only data for grains of mean diameter $d_{g}=114 \mu \mathrm{m}$ are shown. 



\section{Droplet impact near a millimeter-size hole}

\section{॥}

We investigate drop impact dynamics near closed pits and open-ended pores experimentally. The resulting impact phenomena differ greatly in each case. For a pit, we observe three distinct phenomena, which we denote as a splash, a jet and an air bubble, whose appearance depends on the distance between impact location and pit. Furthermore, we found that splash velocities can reach up to seven times the impact velocity. Drop impact near a pore, however, results solely in splashing. Interestingly, two distinct and disconnected splashing regimes occur, with a region of planar spreading in-between. For pores, splashes are less pronounced than in the pit case. We state that, for the pit case, the presence of air inside it plays the crucial role of promoting splashing and allowing for air bubbles to appear.

This chapter is published as: Rianne de Jong, Oscar R. Enríquez, and Devaraj van der Meer, "Exploring droplet impact near a millimetre-size hole: comparing a closed pit with an open-ended pore", J. Fluid Mech. 772, 427-444 (2015). 


\subsection{Introduction}

Droplet impact can be observed regularly in daily life, for example, when raindrops hit the ground or while washing the dishes. Additionally, in industrial processes such as spray coating, spray cooling and inkjet printing, drop impact is of major importance. The topic has been studied for more than a century, starting with Worthington, who in 1876 listed the huge variety in shapes that the drop can take after impact on a solid substrate [28]. It has been found that the liquid properties, the droplet size and velocity, the wettability and roughness of the substrate, as well as the surrounding gas pressure, all have an influence on the behavior of the drop after impact. For example, under different conditions the lamella spreading may lead to prompt splashing, corona splashing and fingering [30, 39, 46, 99, 102, 106, 128].

Despite of the fact that many real surfaces are likely to be rough and inhomogeneous, droplet impact research has mostly concentrated on homogeneous isotropic solid surfaces. However, some authors have looked at smooth solid substrates containing a single obstacle [129-133], such as the impact of a droplet near an open-ended hole (pore), revealing different flow behaviour depending on the impact location. For a centred impact, the inner part of the droplet will enter the pore and the remaining liquid will spread [129, 130]. If the droplet falls beside a pore on a wetting substrate, the liquid will be slightly deflected downwards as it spreads over the pore and a splash is created at the outer edge [132]. This is a localized splash, which differs from lamella splashing (e.g., prompt or corona splashing [30, 102]).

Just as in the previous examples, our interest is in droplet impact on a smooth surface containing a hole. The difference is that we focus on impacts on closed holes (pits) of varying diameter and depth which we experimentally investigate and compare to the behavior for impacts on (open-ended) pores. A pit will induce significantly different flow patterns through the interaction of the liquid with both the bottom and the air inside it. This gives rise to richer phenomena than in the pore case. Furthermore, we will address the different regimes by varying the distance of impact to the hole in small steps.

We have structured this chapter as follows. In Sec. 6.2, we introduce the experimental setup in detail. In Sec.6.3 and Sec.6.4, we describe our experimental observations for impact near pits and pores respectively. In addition, Sec.6.4 provides a comparison between impact near pit and pore. Finally, Sec.6.5]summarises our findings. 

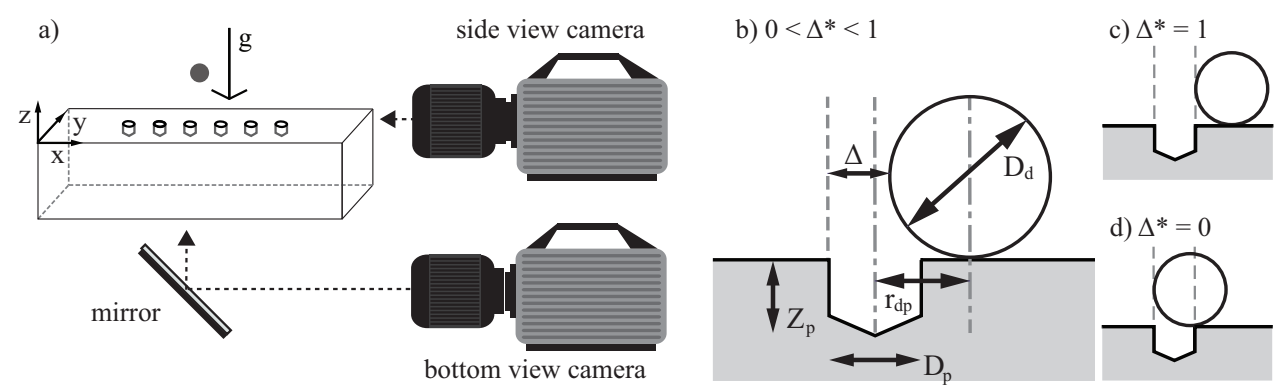

Figure 6.1: a) Schematic representation of the impact of a droplet on a plate with a row of holes, in this case closed pits. The impact is recorded simultaneously by both a side view camera and a bottom view camera with help of a mirror. $b$ ) The relevant parameters are denoted: droplet diameter $D_{d}$, pit diameter $D_{p}$, pit depth $Z_{p}$, the distance between the centers of pit and droplet, $r_{\mathrm{dp}}$, and the distance between the outer pit edge and the nearest droplet edge, $\Delta$. The latter can be normalized with $D_{p}$ to a new parameter $\Delta^{*}$. All parameters are the same for a pore. c) No overlap occurs when $\Delta^{*} \geq 1$. d) There is complete overlap at $\Delta^{*}=0$.

\subsection{Experimental details}

To study the effect of droplet impact near a hole, we use the experimental setup illustrated in figure 6.1 $a$. Droplets are generated by expelling liquid from a syringe at a low rate $(\leq 0.4 \mathrm{ml} / \mathrm{min})$, through a pipe and into a capillary needle (outer diameter $0.85 \mathrm{~mm}$ ). The droplet formed at the needle tip detaches as soon as the gravitational force overcomes that of surface tension. We use milli-Q water (density $\rho=998 \mathrm{~kg} / \mathrm{m}^{3}$, surface tension $\sigma=73 \mathrm{mN} / \mathrm{m}$ and viscosity $\mu=1.0 \mathrm{mPas}$ ) with about $0.5 \%$ in volume of red food dye added to increase contrast (without changing the surface tension). With these properties and the fixed needle size, the droplet diameter equals $D_{d} \approx 3.0 \mathrm{~mm}$. Unless stated otherwise, the impact velocity $U_{i} \approx 3.0 \mathrm{~m} / \mathrm{s}$ and therefore the Weber number is given by $\mathrm{We}=\frac{\rho D_{d} U_{i}{ }^{2}}{\sigma} \approx 370$ and the Reynolds number is $\operatorname{Re}=\frac{\rho D_{d} U_{i}}{\mu} \approx 9 \times 10^{3}$. Hence, all experiments are performed in the inertial regime and no prompt or corona splashing occurs (the threshold velocity for corona splashing is $4-5 \mathrm{~m} / \mathrm{s}$ for water, We $=650-1050$ [47]).

The target surface is a perspex plate containing a row of six equidistant holes, with a distance between the hole centers of $5.0 \mathrm{~mm}$ which is sufficient to prevent that the flow at one hole affects the flow at the other one. The milli-Q water has a contact angle on the substrate of $70^{\circ} \pm 10^{\circ}$. The pits and pores were made with a drill which has a tip angle of $118^{\circ}$, such that the bottom of the pit has a slight angle 
Table 6.1: Overview of the investigated pit sizes.

$\begin{array}{rlllll}\text { Diameter } D_{p}[\mathrm{~mm}] & 2.0 & 2.0 & 2.0 & 1.0 & 1.5 \\ \text { Depth } Z_{p}[\mathrm{~mm}] & 0.5 & 2.0 & 4.0 & 1.0 & 1.5\end{array}$

as well (figure 6.1). The edge of the holes is sharp, of about $90^{\circ}$, and due to the drilling process, the edge can be slightly rough on a scale of $0.1 \mathrm{~mm}$. For the pit dimensions (table6.1) we selected a large diameter-to-depth aspect ratio (2.0/0.5), a small one (2.0/4.0) and one of order unity (2.0/2.0). To also assess the importance of the absolute size (or pit-to-droplet-diameter ratios), we selected several different pits, while keeping the aspect ratio fixed at unity (1.0/1.0), (1.5/1.5) and (2.0/2.0). The depth is measured at the pit center. We tested one pore (i.e., a hole that penetrates through the entire substrate) with a diameter $D_{p}$ of $2.0 \mathrm{~mm}$ and a length of $10 \mathrm{~mm}$.

Two relevant geometrical dimensionless parameters can be denoted for the substrate: $D_{d} / D_{p}$ and $D_{d} / Z_{p}$. Regarding the first one, if $D_{d} / D_{p} \gg 1$, we speculate that the spreading droplet will be hardly affected by the pit. It will act as small-scale roughness, and can enhance prompt splashing [102, 134]. For $D_{d} / D p \ll 1$, the droplet will just spread inside the pit and if impact energy is sufficient, splash at the edges of it (see [135]). Therefore, we chose $D_{d} / D_{p}$ in this chapter always slightly larger than 1, i.e., $D_{d} \geq D_{p}$. The other parameter $D_{d} / Z_{p}$ varies between $0.75-6$ (pit) and for the pore case, it goes effectively to 0 . This parameter is of interest as it gives the ratio of droplet volume to the volume of air enclosed in the pit, and therefore indicates the relative importance of the role of air.

The key parameter varied in this chapter, $\Delta$, is given by the distance between the outer pit edge and the nearest drop edge, see figure 6.1 b. To explore $\Delta$ in a uniform way for the various pit diameters, we normalize it by the pit diameter $D_{p}$ and denote this dimensionless distance as $\Delta^{*}$ :

$$
\Delta^{*}=\frac{\Delta}{D_{p}}=\frac{r_{\mathrm{dp}}+D_{p} / 2-D_{d} / 2}{D_{p}}
$$

where $r_{\mathrm{dp}}$ is the distance between the centers of droplet and pit. Figures $6.1 b-d$ provide a schematic explanation of the significance of this quantity $\Delta^{*}$. The region $0<\Delta^{*}<1$ corresponds to a partially covered pit at the moment of droplet impact. When $\Delta^{*}$ is greater than 1 , the droplet falls beside the pit. At a negative $\Delta^{*}$, the droplet completely covers the pit. Hence, this dimensionless $\Delta^{*}$ contains information about both inner and outer pit edges, which is relevant for the phenomena we observe. This information would be lost if $\Delta$ were scaled with the droplet diameter. In our experiments, $\Delta^{*}$ is adjusted between -0.4 and 1.3 with small increments. 


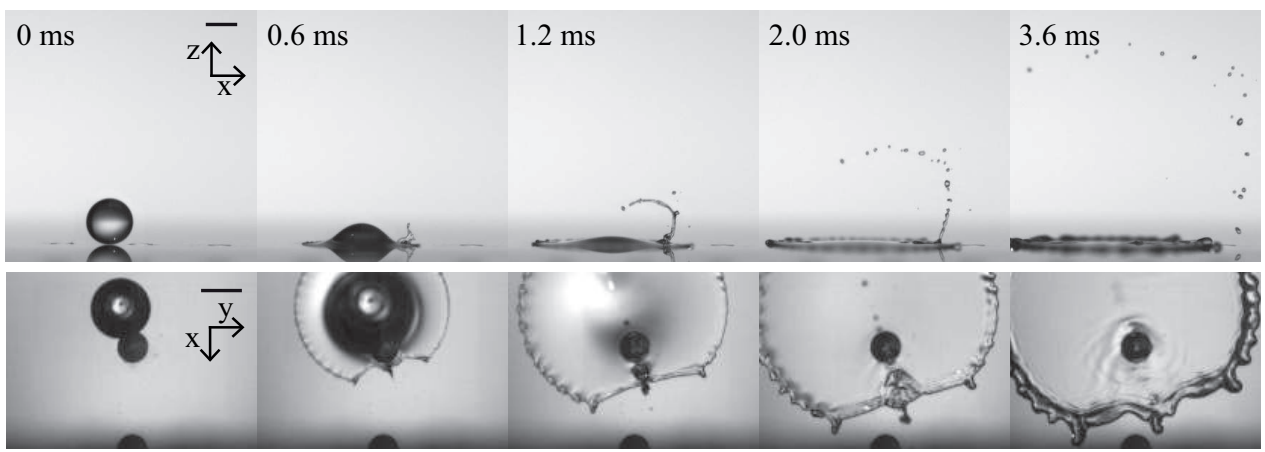

Figure 6.2: Time evolution of a splash imaged from the side (top) and from below (bottom). The droplet impacts at a dimensionless distance $\Delta^{*}=0.89$ from a pit with a diameter $D_{p}=1.5 \mathrm{~mm}$ and a depth $Z_{p}=1.5 \mathrm{~mm}$. At $t=0.6 \mathrm{~ms}$ the splash is created by the deflection of liquid at the outer pit wall. The splash velocity is $4.8 \mathrm{~m} / \mathrm{s}$, averaged over the first millisecond. Droplet diameter $D_{d}$ and velocity $U_{i}$ are $2.9 \mathrm{~mm}$ and $2.93 \mathrm{~m} / \mathrm{s} \mathrm{respec}$ tively. The bar in the two leftmost figures represents $2 \mathrm{~mm}$. Note that the orientation of the side and bottom view differs by $90^{\circ}$, see figure 6.1 for the coordinate system.

The impact events are recorded simultaneously by high-speed cameras from the side (Photron SA1.1) and from below (pits: Photron APX-RS, pores: Photron SAX2) at a frame rate of $5-10 \mathrm{kHz}$ (figure 6.1 a). Additional high-speed imaging experiments are performed by zooming in from below with a long-distance microscope to capture the flow behavior inside the pit (Photron SA1.1). The droplet diameter $D_{d}$, impact velocity $U_{i}$ and the distance between the location of impact and the pit/pore center, $r_{\mathrm{dp}}$, are determined from the recordings.

\subsection{Impact near pits}

\subsubsection{Observed phenomena}

Droplet impact near a pit may result in any of the following three phenomena: (i) a splash, where spreading liquid is partly diverted at the outer pit edge into the vertical direction; (ii) a jet, where some liquid is expelled from the pit at the inner pit edge in an almost exclusively vertical direction; and (iii) an air bubble, which is seen to emerge from the pit. More details on the phenomena are given in table 6.2 Figures 6.2 and 6.3 present a series of side and bottom view images extracted from the recordings. 
Table 6.2: Overview and definitions of various phenomena.

\begin{tabular}{|c|c|c|}
\hline Phenomenon & Hole type & Description \\
\hline Jet & Pit & $\begin{array}{l}\text { A jet emerges at the inner edge, i.e., the edge closest to } \\
\text { the point of impact, and originates from liquid that en- } \\
\text { ters the pit and is deflected upwards by the presence of } \\
\text { a bottom (and side walls). In time, a jet always occurs } \\
\text { after a splash. }\end{array}$ \\
\hline Air bubble & Pit & $\begin{array}{l}\text { An air bubble appears for almost full overlap between } \\
\text { droplet and pit caused by a pressure build-up inside the } \\
\text { latter. }\end{array}$ \\
\hline Splash & Pit or pore & $\begin{array}{l}\text { A splash is created as spreading liquid hits the outer } \\
\text { edge of pit or pore, which acts as an obstacle to the } \\
\text { flow. It is observed for all pit experiments (as long as } \\
\Delta^{*}<1.5 \text { ) and always happens before a jet or an air bub- } \\
\text { ble. }\end{array}$ \\
\hline Slow splash & Pore & $\begin{array}{l}\text { A slow splash occurs for } 1.0<\Delta^{*}<1.5 \text { and has most } \\
\text { likely the same origin as the pit splash. It is broad and } \\
\text { moves inwards in the horizontal direction. }\end{array}$ \\
\hline Thin splash & Pore & $\begin{array}{l}\text { A thin splash also appears at the outer edge, but for } \\
0.5<\Delta^{*}<0.7 \text {. It has less momentum than the pit } \\
\text { splash (under the same conditions), is thin and shoots } \\
\text { upwards. Notice that for } 0.7<\Delta^{*}<0.85 \text {, there is no } \\
\text { splash. This marks the distinction between the slow } \\
\text { and the thin splash. }\end{array}$ \\
\hline Blob & Pore & $\begin{array}{l}\text { A blob is a slow splash, however, only visible for a few } \\
\text { frames and with a negligible velocity. It appears in the } \\
\text { region } 0.85<\Delta^{*}<1.0 \text {. }\end{array}$ \\
\hline
\end{tabular}


a) Splash and jet

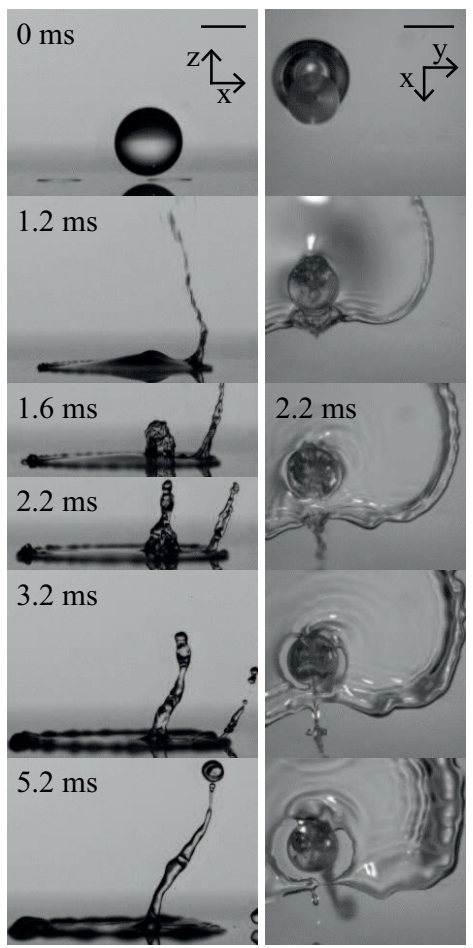

b) Splash and air bubble

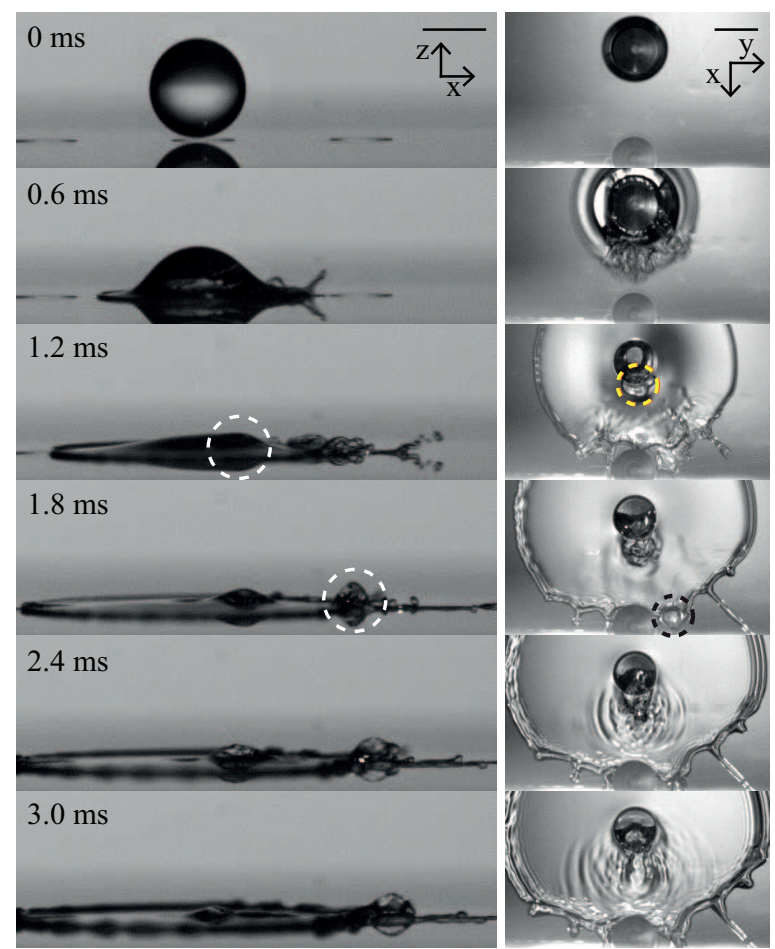

Figure 6.3: Snapshots of two distinct phenomena: The formation of a jet $a$ ) and the appearance of an air bubble $b$ ) imaged from the side (left) and from below (right). The bar in the top figures represents $2 \mathrm{~mm}$. Note that the orientation in side and bottom view differs by $90^{\circ}$. For both cases the parameters are as follows: $D_{p}=2.0 \mathrm{~mm}, Z_{p}=2.0 \mathrm{~mm}, D_{d}=3.1$ $\mathrm{mm}, U_{i}=2.99 \mathrm{~m} / \mathrm{s}$. $a$ ) For a dimensionless distance of $\Delta^{*}=0.16$, first a fast splash appears at $t=1.2 \mathrm{~ms}$, and subsequently a jet is observed at $t=1.6 \mathrm{~ms}$. The velocities in the first millisecond are $17.7 \mathrm{~m} / \mathrm{s}$ for the splash and $2.8 \mathrm{~m} / \mathrm{s}$ for the jet. $b$ ) For full overlap between droplet and pit $\left(\Delta^{*}=-0.25\right)$, the resulting phenomenon is totally different. Air is pushed out of the pit by the impacting water. An air bubble is visible at the pit edge at time $t=1.2$ $\mathrm{ms}$ and another air bubble is observed at the spreading edge from $t=1.8 \mathrm{~ms}$ onward. The first is the air bubble we will focus on in this chapter. Both are indicated with dashed circles. 


\section{Splash}

In figures 6.2 and 6.3 we can see examples of splashes forming as spreading liquid impacts on the outer edge of the pit, with which we denote the spot that is most distant from the droplet's impact location. Although this phenomenon is observed for the whole range of $\Delta^{*}$ explored, the direction and velocity of the splash varies. For small overlap (figure 6.2), the splash is thin, curved inwards and its speed is comparable to the impact velocity. As overlap increases (figure6.3 $a$ ), the splash becomes faster and more vertical. For full overlap (figure 6.3b), it is broad, slow and moving mainly horizontally. The splash velocity is discussed in detail in Sec. 6.3.3. From the high-speed imaging experiments capturing the flow inside the pit (figure 6.4a), we found that liquid is also deflected downwards and that some will remain in the pit after the impact event. The flow is captured in a simple schematic in figure $6.6 a$.

\section{Jet}

A jet is only created when the impacting droplet overlaps with the pit. The necessary amount of overlap depends on the pit depth. In figure 6.3 $a\left(\Delta^{*}=0.16\right)$ two phenomena occur: first, a splash at the outer edge (at $t=1.2 \mathrm{~ms}$ ) and subsequently, a thicker object appears at the inner edge, which we denote as a jet. In this case, there are two competing flow mechanisms: on the one hand, the spreading liquid that leads to splashing as described above; on the other hand, as there is overlap between droplet and pit, part of the liquid can immediately enter.

By what mechanism is the jet created? The flow inside the pit cannot be directly extracted from the close-up views in figure 6.4c. For pits of $4.0 \mathrm{~mm}$ in depth, however, the flow is visible on the side view images, see figure 6.5 As the pit is not totally in focus, the air-water interface is indicated by a dashed line. The extracted flow, which is also illustrated in figures $6.6 \mathrm{c}-e$, is as follows: after impact, the liquid will spread outwards and hit the outer edge, resulting in an upward and a downward splash. The latter will continue on the pit bottom and will be deflected upward at the inner edge, resulting in a jet. The location where the jet appears, indicates that the outward flow dominates the downward one. This flow path is consistent with the observed jet emergence times, which will be discussed in Sec.6.3.4.

\section{Air bubble}

Another observed phenomenon, an air bubble, is found for large overlap $\left(\Delta^{*} \approx 0\right)$. Here we show the case for which $\Delta^{*}=-0.25$ (see figure $6.3 \mathrm{~b}$ ). The air bubble is vis- 
a)
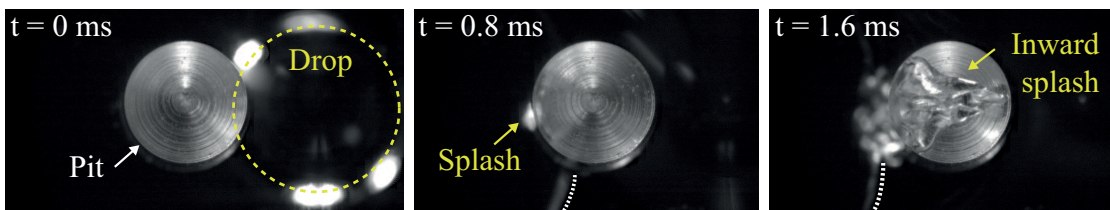

b)
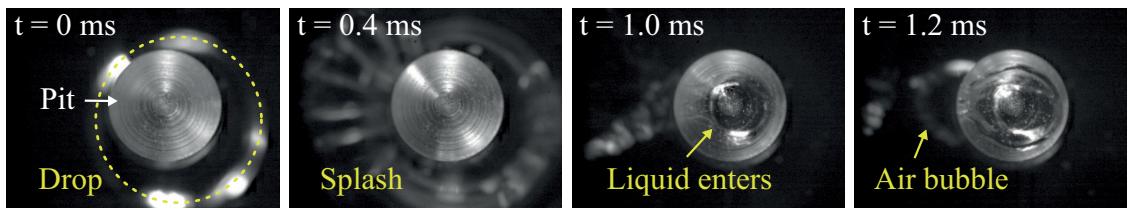

c)
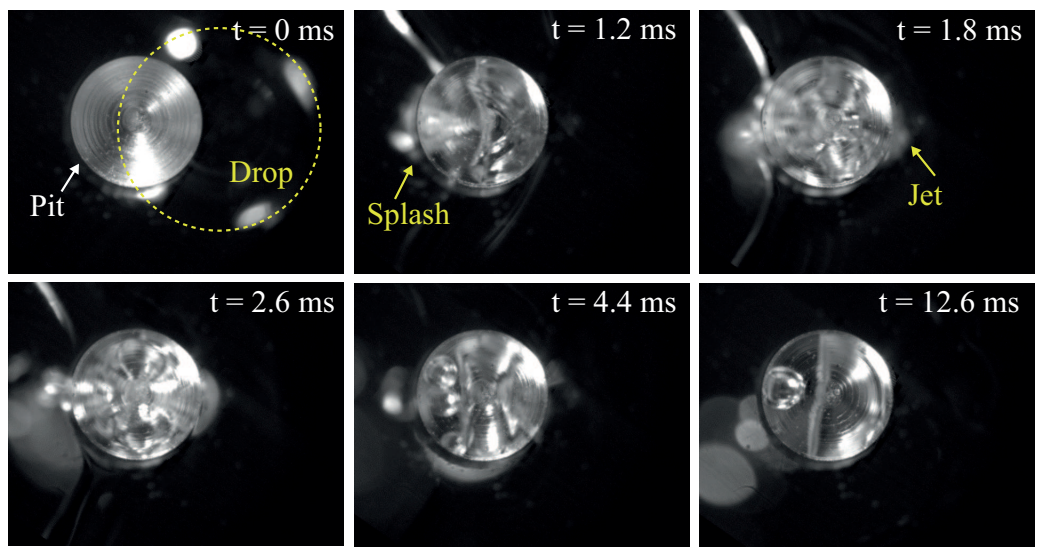

Figure 6.4: Images showing the flow inside a pit of $2.0 \mathrm{~mm}$ in both diameter and depth. a) For small overlap between droplet and pit $\left(\Delta^{*}=0.83 \pm 0.05\right)$, liquid enters the pit at the outer edge, resulting in an inward flow. $b$ ) For full overlap $\left(\Delta^{*}=-0.06 \pm 0.05\right)$, after liquid enters the pit, an air bubble emerges. c) For partial overlap between drop and pit $\left(\Delta^{*}=0.47 \pm 0.05\right)$, the flow behavior is more complex, as liquid and air mix. However, a splash can be seen at the outer edge and a jet at the inner one (as can also be observed in figures $6.3 a$ and 6.5 . 


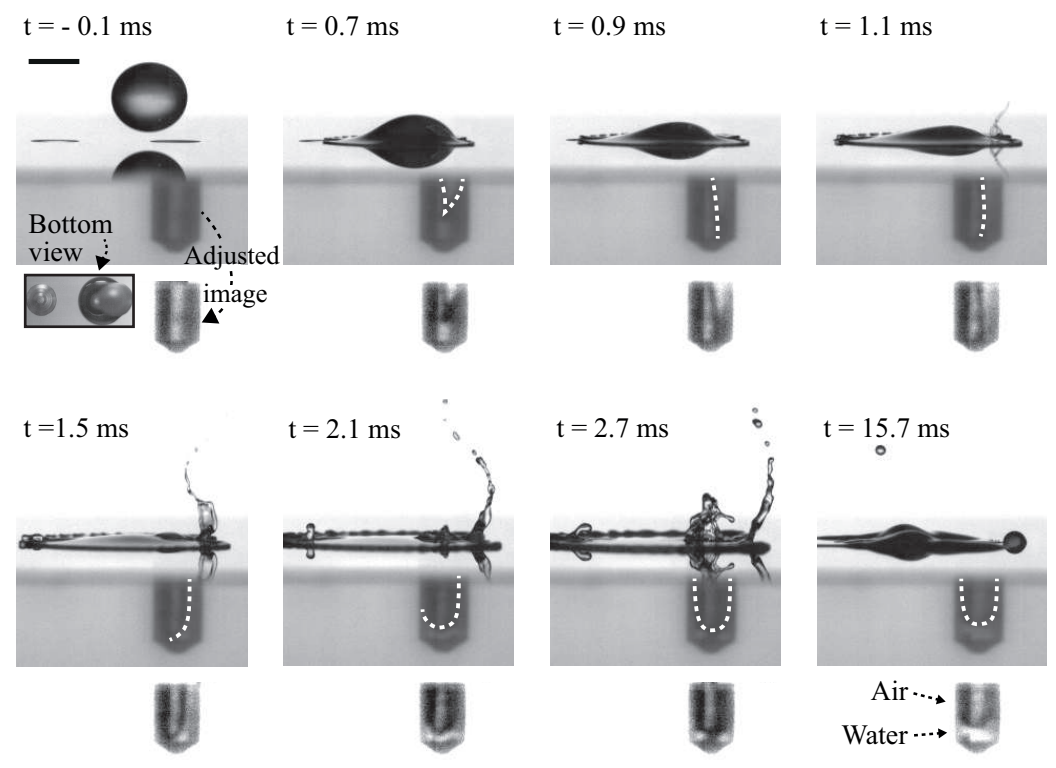

Figure 6.5: For partial overlap $\left(\Delta^{*}=0.22\right)$, the liquid enters at the outer side of the pit, is deflected upwards by the bottom, after which a jet appears at the inner edge. Each frame shows the spreading droplet on top of the substrate as well as the flow inside the pit, with an extra picture for the flow inside the pit with enhanced brightness and contrast directly below it. Pit diameter and depth are 2.0 and $4.0 \mathrm{~mm}$ respectively (The pit on the left is shallow in depth, and hence not visible from the side). The inset in the first figure gives the bottom view at the moment of impact, showing that the droplet impacts in line with the pits and hence in the plane of the side view camera. The scale bar represents $2.0 \mathrm{~mm}$. Other parameters are as follows: $D_{d}=3.0 \mathrm{~mm}, U_{i}=3.03 \mathrm{~m} / \mathrm{s}$ and $U_{s}=9.2 \mathrm{~m} / \mathrm{s}$. 


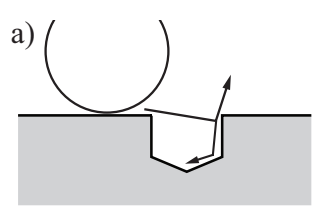

c)

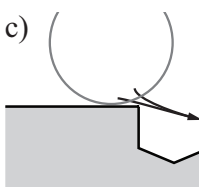

d)

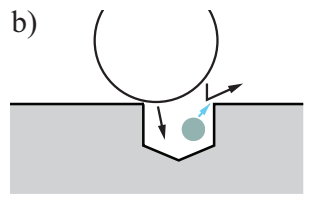

e)

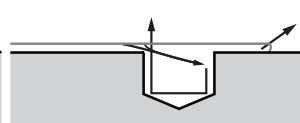

Figure 6.6: A schematic representation of the (possible) flow shortly after impact as inferred from high-speed images taken from below with a long-distance microscope focusing on the pit (see figure 6.4. a) For little overlap, liquid spreads outwards, hits the outer pit edge and is deflected both upward and downward. b) For full overlap, liquid flow is separated: a broad splash outwards (right arrow) and liquid entering the pit near the inner pit edge. This liquid pushes out an air bubble at the outer pit edge (filled circle). $c$ )-e) Liquid hits the outer pit edge $c$ ), creating an upward and downward flow $d$ ), resulting in a splash at the outer edge and a jet at the inner one, penetrating the flat $\operatorname{drop} e$ ).

ible at time $t=1.2 \mathrm{~ms}$ at the outer edge. Subsequently, it moves slightly outwards $(t=1.8 \mathrm{~ms}$ ) and breaks up ( $t=3.0 \mathrm{~ms}$ ). From the bottom view images (figure $6.4 b$ ), we can see that the central part of the droplet enters the pit. The corresponding flow is sketched in figure $6.6 b$.

We state that the air bubble is pushed out as a consequence of liquid sealing the pit. The driving mechanism is a pressure build-up due to liquid entering the closed-off pit, i.e., the air inside it gets compressed. There are three pressures to be considered: the dynamic impact pressure, the hydrostatic pressure and the Laplace pressure. The dynamic pressure is already relevant at early times, because it is responsible for liquid entering the pit. Its initial value scales with $\rho U_{i}^{2} \propto 10 \mathrm{kPa}$ and is observed (from simulations) to drop quickly after $\tau=D_{d} /\left(2 U_{i}\right)$ for droplet impact on both a liquid film and a solid substrate [36, 136]: it is one order of magnitude smaller at $t \approx 1.5 \tau$ and two orders of magnitude at $t \approx 3.0 \tau$. In our case, the impact time scale is $\tau=0.5 \mathrm{~ms}$. The hydrostatic pressure applied by the liquid film is $\Delta P_{\text {film }}=\rho g d$, where $g$ is the gravitational acceleration and $d$ is the thickness of the film. Even if the liquid film were $1 \mathrm{~mm}$ thick, the film pressure $\Delta P_{\text {film }}$ would be approximately $10 \mathrm{~Pa}$ and would therefore constitute a negligible obstacle for the air bubble to move upwards. Calculating the Laplace pressure, one finds it to be of the same order of magnitude as the pressure build-up inside the pit $\Delta P_{\text {pit. }}$ Therefore, it will influence the final shape and size of the bubble, but it will not prevent 
the bubble from appearing.

Hence, the air has to get compressed, such that the pressure build-up $\Delta P$ inside the pit overcomes the impact pressure. With Boyle's law we can estimate the volume of compression that is needed: $\Delta V=V_{p} \Delta P /\left(P_{0}+\Delta P\right) \equiv \alpha V_{p}$. The precise value of $\Delta P$ depends on the moment the pit is sealed, i.e., on the droplet velocity at that time. As we show in figure 6.7 air bubbles are observed for large overlap only, and consequently, time of pit closure is rather short. Therefore, using $U_{i}$ is a reasonable estimate: $\Delta P \approx \frac{1}{2} \rho U_{i}^{2}$ and accordingly $\Delta V \approx 0.04 V_{p}$. This indicates that only a small amount of liquid needs to enter the sealed pit, in order to reach a pressure inside it opposing the dynamic pressure.

As the dynamic pressure quickly drops after $t>\tau$, one can expect air bubbles to form slightly afterwards. We observe air bubbles in a time range of $0.8-1.6 \mathrm{~ms}$. Hence, as soon as the liquid has decelerated sufficiently, the pressure build-up inside the pit becomes dominant and thus the appearance of the air bubble is directly related to the amount of liquid that enters the pit: for all practical purposes the air within the pit can be considered to be incompressible.

For almost the whole $\Delta^{*}$ range, there is an asymmetry between the liquid entering the pit and the pit itself, therefore designating where the air bubble appears. However, when the centers of the droplet and the pit are aligned at impact $\left(r_{\mathrm{dp}}=\right.$ 0 ), a Rayleigh-Taylor (or Richtmyer-Meshkov) instability can play a role. Its characteristic acceleration is given by $a^{*}=U_{i}^{2} /\left(D_{d} / 2+\alpha Z_{p}\right)$, where $\alpha$ is the pressure fraction calculated above: $\alpha \approx 0.04$, indicating that the air quickly decelerates the liquid. The unstable wavelengths for water are $\lambda_{\text {unstable }}>2 \pi \sqrt{\sigma /\left(\rho a^{*}\right)} \gtrsim 0.7 \mathrm{~mm}$ for all the investigated pit depths. Therefore, this instability can dominate for head-on impact as it is smaller than the pit diameters, which vary from $1.0-2.0 \mathrm{~mm}$.

\subsubsection{Phase diagram}

All of the phenomena discussed above are plotted in the phase diagram of figure 6.7 which maps their dependence on the dimensionless distance $\Delta^{*}$ and the dimensionless pit depth $Z_{p} / D_{d}$. The broad picture is as follows: Decreasing $\Delta^{*}$ from large to intermediate to small, we move from a regime with a splash only, through one with a splash and a jet, into a region with a splash together with an air bubble. Decreasing the dimensionless pit depth enlarges the range (of $\Delta^{*}$ ) for which a jet is observed, whereas the domain in which an air bubble is found remains approximately constant in size until the pit becomes too shallow to produce an observable bubble or jet.

The transition between splash only and jetting (near $\Delta^{*}=1$ ) suggests that for deeper pits a larger overlap is needed to produce a jet. This is probably related to 


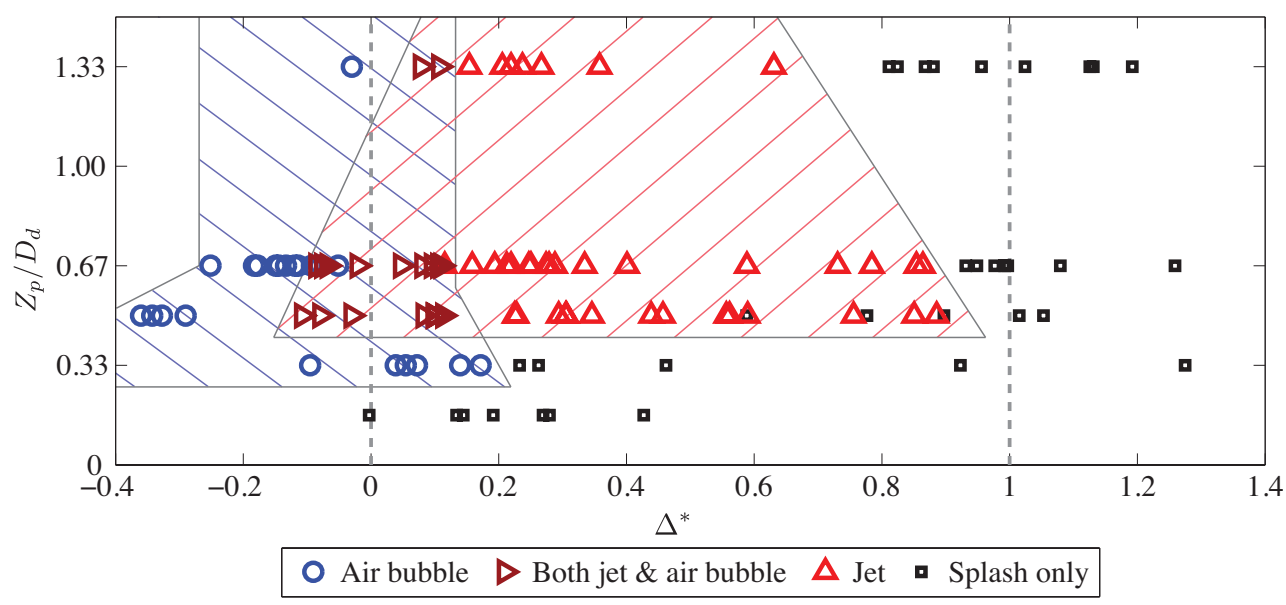

Figure 6.7: Phase diagram of the three different types of phenomena (splash, jet and air bubble) in the parameter space spanned by the dimensionless distance between the outer pit edge and nearest drop edge $\Delta^{*}$ and the dimensionless pit depth $Z_{p} / D_{d}$. All experiments are done in the inertial regime for a Weber number of We $\approx 370$. The shaded regions and their boundaries are guides for the eye: the left one $(\backslash \backslash)$ represents the region where an air bubble appears, the right shaded area ( / / ) shows the region where a jet is present. The droplet diameter and velocity are kept constant within a $3 \%$ accuracy to $3.0 \mathrm{~mm}$ and $2.95 \mathrm{~m} / \mathrm{s}$, respectively. Note that a splash is observed in all cases, albeit sometimes small.

the longer travel path $\left(2 Z_{p}+D_{p}\right)$ before the liquid can exit a deeper pit (i.e., as a jet; figure 6.6 $c-e$ ). There are two lines of reasoning regarding the relation between depth and jet formation. First, a long travel path has more dissipation than a short one, and second, as a longer path will take a longer time, there can be a larger droplet mass above the liquid in the pit, preventing it from leaving. On the other side of the regime, near $\Delta^{*}=0$, where jetting gives way to the formation of a bubble only, the reasoning is similar. Again, we think that the disappearance of the jet, that occurs for larger $\Delta^{*}$ as the pit depth increases, is related to the path that the liquid needs to travel. In addition, for full overlap $\left(\Delta^{*}<0\right)$, a jet is noticed at the outer edge, appearing after the splash.

The air bubble regime is bound between $\Delta^{*} \approx 0.1$ and the minimum value of $\Delta^{*}$, where the latter by its definition increases with increasing pit diameter, causing the blank region in the top left of figure 6.7 where no measurements are possible for the pits we have used. We found that the location of the first-mentioned boundary (around $\Delta^{*} \approx 0.1$ ) solely depends on the absolute distance $\Delta$, i.e., the transition is invariant of the pit diameter and depth. For all pit sizes tested, the 
transition occurs at $\Delta=0.22 \mathrm{~mm}$. We believe that the invariance for the pit diameter $D_{p}$ relates to the spreading front of the droplet near to this critical $\Delta$. The spreading liquid overcomes the disruption by the pit for this small distance and encloses the pit quickly from both sides. In addition, it is independent of pit depth, because the pressure build-up inside the pit is sufficient for any of the pit depths (see Sec.6.3.1 for details); it suffices to have a trapped amount of air. Note that the jet regime and air bubble regime partly coincide $\left(\Delta^{*} \approx 0.1\right)$. It means that there is a slow transition from mainly outward spreading liquid (figures $6.6 c-e$ ) to liquid that mainly enters the pit (figure 6.6b).

Finally, we speculate on the changes to the phase diagram of figure 6.7 if we were to increase the drop size. Keeping $\Delta^{*}$ constant, this would increase the mass flow towards and inside the pit, and therefore it can be expected that the regime where jets are observed would be slightly extended towards higher values of $\Delta^{*}$. We do not anticipate a similar change for the air bubble regime, since the liquid volume entering the pit does not depend on the droplet size (cf. Sec.6.3.1).

\subsubsection{Splash velocity}

Figure 6.8 $a$ shows a plot of the splash velocity against $\Delta^{*}$ for various pit sizes. First, we can observe that for large distance $\left(\Delta^{*} \gtrsim 1\right)$ the splash velocity is close to the impact velocity. Second, the splash velocity increases rapidly with decreasing $\Delta^{*}$, until a maximum is reached of seven times the impact velocity at $\Delta^{*} \approx 0.2$. Third, beyond that maximum, the splash velocity $U_{s}$ decreases with decreasing distance. The splash velocity was measured always within $1.0 \mathrm{~ms}$ from its first appearance and was obtained by averaging over $1.0 \mathrm{~ms}$ to diminish effects of shape fluctuations of the tip droplet. In very few cases, the averaging was done for a shorter time period, typically when splash velocities were small. For fast splashing, air drag will have an effect on the droplets, as the droplets are far above their terminal velocity, especially when they are small [137]. However, this velocity reduction in the averaging time is within our measurement error. Figure $6.8 \mathrm{~b}$, for a pit of 2.0 $\mathrm{mm}$ in both diameter and depth and different impact velocities, indicates that the splash velocity can be normalized with the impact velocity. Some authors have proposed that $U_{s} \sim U_{i}^{3 / 2}$ for another type of splashing, namely the appearance of micro-droplets at the first instant of prompt splashing [137, 138]. Such a scaling does not lead to a better collapse of the data. (For the above, note that this is the only instance in this chapter where the impact velocity is varied.)

As we consider the behavior of the individual pit sizes in figure $6.8 a$, we notice that to reach high splash velocities a pit depth of $1.5-2.0 \mathrm{~mm}$ is optimal (filled symbols). For a deeper pit of $4.0 \mathrm{~mm}, U_{s}$ levels off $(\diamond)$, which is also the case for the 


\subsection{IMPACT NEAR PITS}
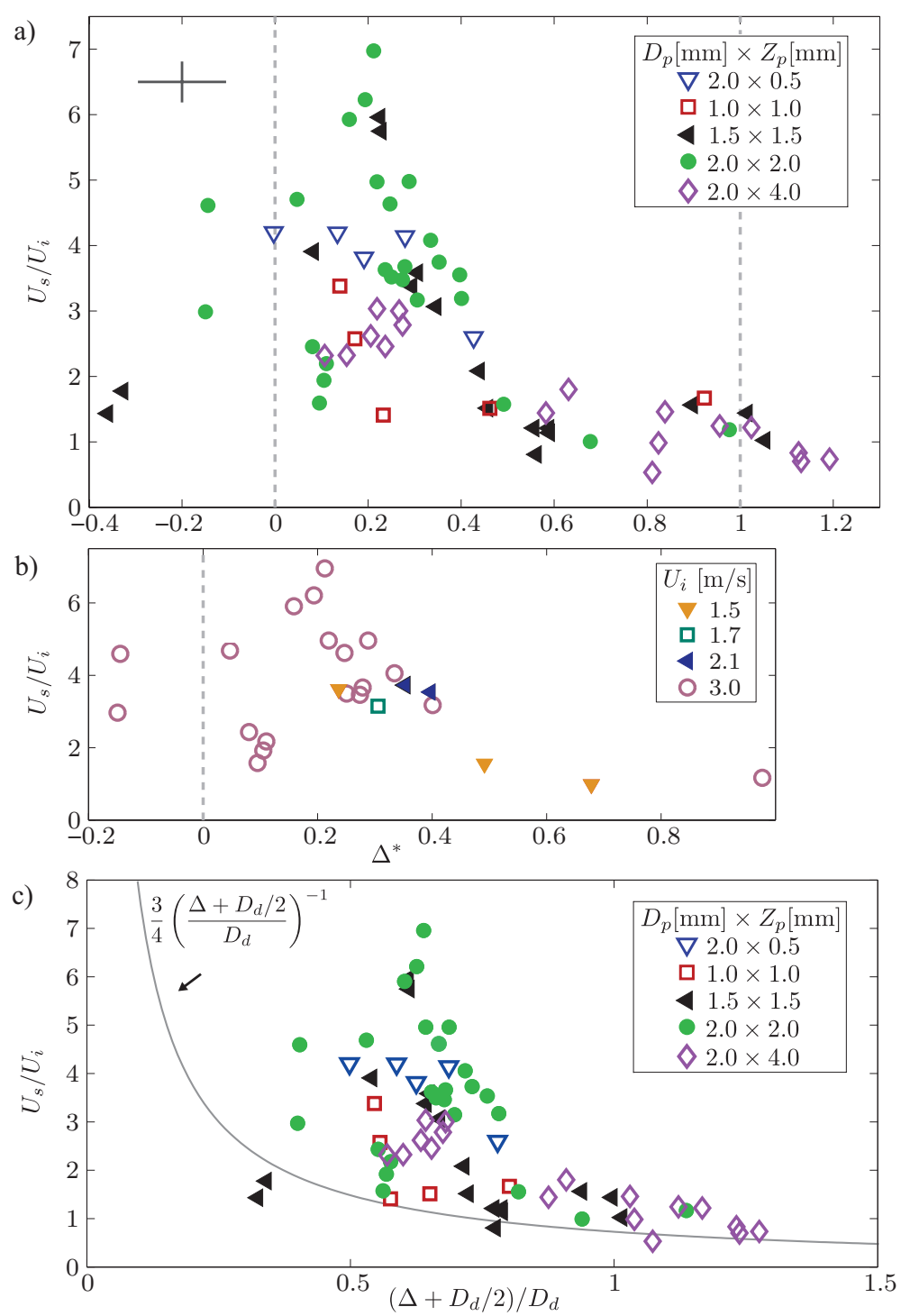

Figure 6.8: $a$ ) The dimensionless splash velocity $U_{s} / U_{i}$ as a function of the dimensionless distance $\Delta^{*}$ for various pit sizes. For decreasing distance $\Delta^{*}$ the splash velocity $U_{s}$ increases up to a maximum of $7 U_{i}$ at around $\Delta^{*}=0.2$. An average error bar is given at the top left. $b$ ) A selection of the data of $a$ ), for $2.0 \mathrm{~mm} \times 2.0 \mathrm{~mm}$ pits, with various symbols indicating the different impact velocities. $c$ ) The dimensionless splash velocity $U_{s} / U_{i}$ is now shown as function of the dimensionless distance between droplet center and pit edge, $\left(\Delta+D_{d} / 2\right) / D_{d}$. The solid line is related to the lamella spreading theory of [47]. 
shallowest pit $(\nabla)$. One outlier is the pit of $1.0 \mathrm{~mm}$ in both diameter and depth $(\square)$, because the splash velocity only increases from $\Delta^{*} \leq 0.2$, which is, interestingly, also the critical $\Delta^{*}$ for the transition to air bubbles for this pit size. For all other pits, the upward trend in velocity starts from $\Delta^{*} \approx 0.5$. For each pit size, the data is consistent within its measurement uncertainties, except for a pit depth of $2.0 \mathrm{~mm}$ $(\bullet)$ at $\Delta^{*} \approx 0.1$. We believe that the main flow behavior at this $\Delta^{*}$ is very sensitive to small fluctuations during impact, since this $\Delta^{*}$ domain is a transition region, in which both a jet and an air bubble occur.

\section{Splash velocity related to lamella spreading}

For droplet impact on solid substrates, the spreading of the lamella in time has been widely studied [47, 139, 140]. A scaling for this spreading radius in time is easily obtained with simple dimensional analysis. However, an exact prediction had only recently been obtained [47], which matches well with lamella spreading experiments. By using potential flow theory combined with Wagner's theory [141], Riboux \& Gordillo [47] find that the spreading of the wetted area goes as $R /\left(D_{d} / 2\right)=\sqrt{3 t U_{i} /\left(D_{d} / 2\right)}$. This can be related to our experiments by looking at the distance between the droplet center and the pit edge, which is $L \equiv \Delta+D_{d} / 2$ (see figure 6.1 b). We can try to relate $L$ to the splash velocity, by first taking its time derivative: $d L / d t=\sqrt{3 D_{d} U_{i} /(8 t)}=3 D_{d} U_{i} /(4 L)$. If $U_{s}$ scales with $d L / d t$, the derivation gives $U_{s} / U_{i} \sim(3 / 4) /\left(L / D_{d}\right)$. Note, however, that the theory of Riboux \& Gordillo [47] pertains to the spreading of the circular wetted area and not to the faster ejecta spreading. Hence, a slightly larger pre-factor should be expected.

We show the prediction in figure 6.8k, plotting the dimensionless splash velocity versus $L / D_{d}=\left(\Delta+D_{d} / 2\right) / D_{d}$. Interestingly, the data follows this scaling for $L / D_{d}>0.8$. However, especially for the fast splashes (solid symbols), the data clearly deviates from lamella spreading, suggesting a different mechanism. We think that the relevant difference in experiments with various pit sizes, is the amount of air initially in the pit and the resulting outward air flow as the liquid enters into it.

\subsubsection{Time until jet formation}

The jet is caused by liquid that enters the pit (mainly) at the outer edge and travels a path $s_{\mathrm{jf}} \simeq 2 Z_{p}+D_{p}$ to appear at the inner pit edge, see figure6.6for a sketch. We measured the time between impact and jet formation, $t_{\mathrm{j} f}$, and show it in figure $6.9 a$ as a function of $\Delta^{*}$. The jet formation time is always found to be longer for larger pits. When it is normalized with $s_{\mathrm{j} f} / U_{i}$, the data collapses nicely, see figure 6.9 $b$. 

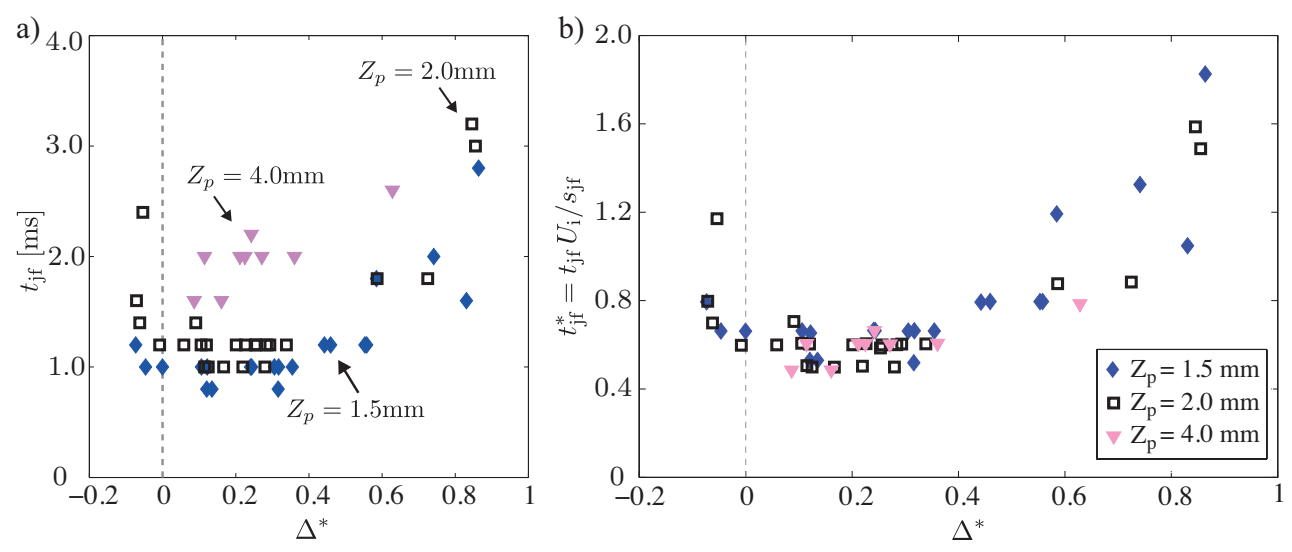

Figure 6.9: $a$ ) The time that it takes for a jet to shoot out from the moment of impact, $t_{\mathrm{jf}}$ as a function of the dimensionless distance $\Delta^{*}$ for three different pit depths $Z_{p} . b$ ) The nondimensional jet formation time (scaled with the impact velocity $U_{i}$ and the travel path $s_{\mathrm{jf}}$ ) as a function of $\Delta^{*}$. The data collapses nicely.

Furthermore, we note that the proper velocity to collapse the jet time, $t_{\mathrm{j}}$, is the impact velocity, rather than the splashing velocity. In the region $0<\Delta^{*}<0.4, t_{\text {jf }}$ hardly changes, in contrast to the splashing velocity (figure $6.8 a$ ), which changes rapidly in this regime. Moreover, the splashing velocity reaches significantly different values when depth is varied.

\subsection{Impact near a pore}

\subsubsection{Observed phenomena}

Impact near an open-ended pore results in a splash or no splash, i.e., neither jets nor air bubbles are observed. This stands to reason in view of the mechanisms by which the latter two are formed: the jet and air bubble need a bottom wall and an enclosed cavity with air for their formation respectively. However, three distinct splash types are observed: a splash, a thin splash and a blob, all caused by spreading liquid hitting the outer pore edge. See table 6.2 for the splash definitions. Figure 6.10 presents these phenomena as a function of $\Delta^{*}$ along with experimental snapshots. Surprisingly, there are two distinct splashing regimes separated by a region in which planar spreading occurs.

For $1.0<\Delta^{*}<1.5$, we observe a slow splash, which descends into a region with a blob at both $\Delta^{*}<1.0$ and $\Delta^{*}>1.5$, until just planar spreading is seen. Note that 


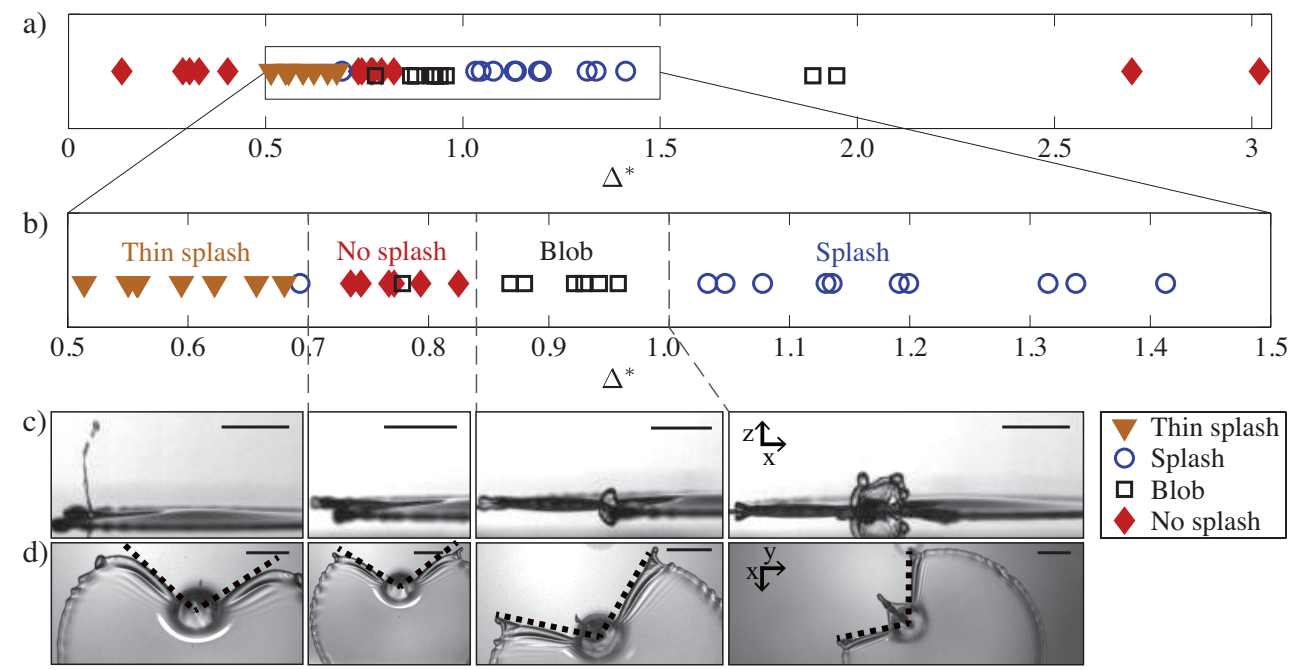

Figure 6.10: The various phases observed when a droplet impacts near an open-ended pore: a splash $(\bigcirc)$, a thin splash $(\nabla)$, a blob $(\square)$ or planar spreading $(\diamond)$. $a$ ) Phase diagram of different kinds of pore splashes. $b$ ) A zoom of $a$ ). $c$ ),d) Images of each phenomenon, with side view $c$ ) and bottom view $d$ ). Snapshots are all taken $1.7 \mathrm{~ms}$ after impact and from left to right $\Delta^{*}$ is given by $0.55,0.74,0.94$ and 1.14 respectively. The bar in each image represents $2.0 \mathrm{~mm}$. Note that the orientation in side and bottom views differs by $90^{\circ}$. The dashed lines in $d$ ) indicate the area near the pore that remains dry throughout spreading.

a blob denotes a splash that is only visible for a short time and has almost no velocity. The slow splash appears at the outer pore edge, but tends to travel sideways rather than upwards. It broadens towards the inner pore edge (see side view image), and remains thin in the other horizontal dimension (see bottom view image). This splash is the one studied by Roisman et al. [132], who restricted themselves to impacts near $1.0 \mathrm{~mm}$ (in diameter) pores for $\Delta^{*}>1$, i.e., for no overlap between droplet and pore, and is simply caused by liquid deflected by the pore edge. In other words, we believe that this splash has a similar origin as the one observed for the pit. The disappearance of the splash for large $\Delta^{*}(>1.5)$ is probably due to the fact that the spreading liquid loses its kinetic energy to surface energy [39]. On the other boundary, $\Delta^{*}<1$, not all downward motion will be transferred to (horizontal) spreading and this motion is not opposed and redirected by the air present in the pore, as in the case of the pit. Therefore, the main flow will become downward, all the way through the pore.

The other splash regime is found in the range $0.5<\Delta^{*}<0.7$, and has sharp 
transitions towards planar spreading at both boundaries. Here we observe thin splashes, which reach higher velocities than the slow splash (see Sec. 6.4.2 and figure 6.11). These thin splashes are created when there is already partial overlap between drop and pore, such that quickly after impact the pore edge is partially wetted. As there is no pressure to oppose it, the liquid at the edge can flow into the pore. Therefore, we conjecture that liquid entering from different sides collides inside the pore, leading to a focused splash at the outer edge.

The intermediate region of $0.7<\Delta^{*}<1.0$, where there is only planar spreading or a small blob visible, suggests that there is a delicate balance between spreading and downward motion. This balance is responsible for the transitions between splashing, spreading and thin splashing. Furthermore, if we compare the thin splash with the splash above a pit for the same $\Delta^{*}$-region, the trend in the velocity, volume, and time of splash formation are all different. Moving to larger overlap, i.e., $\Delta^{*}<0.5$, liquid simply enters into the pore, as also observed by Delbos $e t$ al. [129] for full overlap.

\section{Comparing pit and pore}

The key difference between the pore and the pit is the difference in interaction between liquid and air, which becomes clear when comparing figure 6.10 with figures 6.2 and 6.3. The bottom view images in figure $6.10 d$ show that the pore acts as an obstacle for the spreading liquid, which it does not overcome (at least not within the investigated parameter space). There is always a dry area behind the pore, often with opening angles larger than $90^{\circ}$, measured from the centre of the pore, indicated in figure $6.10 \mathrm{~d}$. For the pit (figures 6.2 and 6.3), the spreading liquid not only encloses the pit, but for small and large overlap clearly overcomes the disruption. As soon as the pit is sealed, the air inside the pit will behave as a kind of substrate over which spreading is possible. For the pore, however, air can always move out at the bottom, and hence there is no resistance to liquid entering.

Furthermore, even when the pit is not yet sealed by the droplet the air will tend to leave it. As the air can be assumed to be an incompressible fluid, the volume of water that enters the pit will be equal to the volume of air leaving. Therefore, the liquid will already be deflected before the pit is closed off. We can make an estimate of a necessary pit depth, for which the air does not come out immediately and thus compressibility effects become relevant. The time scale for the pressure wave to bounce back from the pit bottom is given by $t_{c}=2 Z_{p} / c$, with $c$ the velocity of sound in air, and should be comparable to the impact time $\tau=D_{d} /\left(2 U_{i}\right)=0.5 \mathrm{~ms}$. This results in a pit depth of at least $85 \mathrm{~mm}$ before one may expect any pore-like splash behavior for pits. The difference in air flow explains 


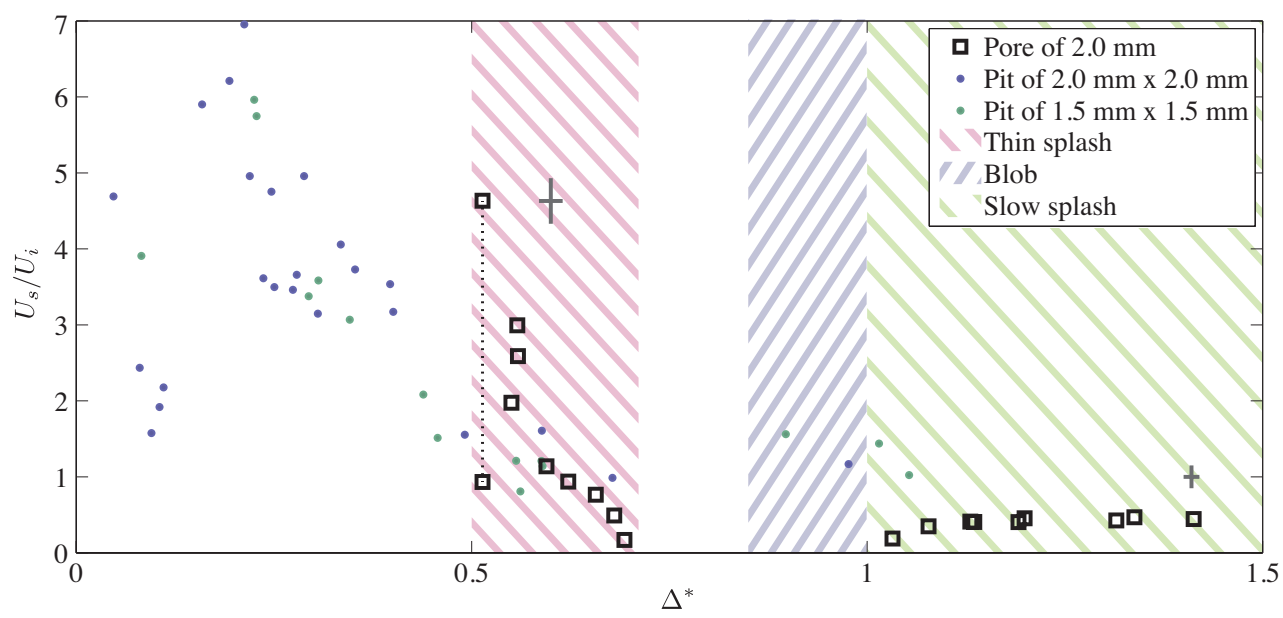

Figure 6.11: The splash velocity normalised by the impact velocity $U_{s} / U_{i}$ versus the dimensionless distance $\Delta^{*}$ for impacts near a pore $(\square)$. The shaded regions are extracted from figure 6.10 to indicate where splashing is expected. The pit data $(\bullet)$ is included as a reference. Error bars are added indicating the typical error in the two splashing regions.

why the splashes for pit and pore differ in velocity (and volume), even in the case that there is no overlap between the impacting droplet and pit or pore (figure 6.2 and last image in figure $6.10 d$ ). Furthermore, it provides a plausible reason that pit splashes are always observed whereas for pores they only occur for a narrow $\Delta^{*}$ range.

\subsubsection{Splash velocity}

The splash velocity in the two splash regions is measured and reported in figure 6.11. In the (slow) splashing regime, the splash velocity is fairly constant around a value of $0.4 U_{i}$. In the thin splashing regime, however, the splash velocity increases with decreasing distance. At $\Delta^{*} \approx 0.5$, two data points are connected by a dashed line. The higher data point corresponds to a fast droplet that shoots out of the pore, of which we could not determine the exact origin, but probably originates from the tip of the splash. A much slower splash appears $0.3 \mathrm{~ms}$ later, represented by the lower data point. The error bars in the figure differ slightly for the two splash regimes. For no overlap, the locations of droplet and pore can be determined with more certainty and the splash fluctuates less, resulting in a smaller error. 
The splash velocity for impacts near pores is, in general, smaller than for impacts near pits. The maximum splash velocity ratio $\left(U_{s} / U_{i}\right)$ is at least 1.5 times smaller, and for the slow splashing regime, i.e., when there is no overlap, it is even 2.5 times smaller. This demonstrates again that the air present in the pit plays a very important role and that it influences both the size of the splashing regime and the splash velocity. There is a small region of $0.5<\Delta^{*}<0.55$ where the thin splash is faster than the pit splash, but overall velocities of the pit splashes are much larger. Furthermore, the splash becomes very thin, and thus has little momentum, again pointing to a different origin for the thin splash in comparison to both the pit splash and the slow splash.

\subsection{Conclusions}

We have found experimentally that the behavior resulting from the impact of a water droplet near a closed pit and an open-ended pore greatly differs. Furthermore, we observed distinct impact phenomena when the amount of overlap between the droplet and the hole is varied (table 6.2).

For impact near a pit these phenomena are, from large to intermediate to small distance $\Delta^{*}$ : a splash only, both a splash and a jet, and both a splash and an air bubble respectively (figures 6.2 and 6.3. Moreover, we found that splashing is most pronounced for pit sizes around $1.5-2.0 \mathrm{~mm}$ in diameter and depth, reaching splash velocities up to seven times the impact velocity, see figure 6.8. The size of the jetting regime, in figure 6.7, clearly narrows as the pit depth is increased, and completely disappears when the depth is infinite (i.e., the pore case).

For impact near pores, we only observe splashing. This stands to reason, since the formation of the jet requires the presence of the flow-deflecting pit bottom and for the emergence of an air bubble an enclosed volume of air is needed, both of which are not present in the case of a pore. Surprisingly, we find two distinct splashing regimes, with horizontal spreading in between (figure6.10). One regime is for $1.0<\Delta^{*}<1.5$, i.e., when there is no overlap between droplet and pore, and for which a slow, sideways splash is observed. The other regime is at $0.5<\Delta^{*}<0.7$, where a thin, fast splash is created, which is less pronounced than for the pit case (see figure 6.11). More research is needed to determine the origin of this splash. We believe the first splash has the same origin as for the pit splash: spreading liquid above the hole is deflected at the outer hole edge.

We state that, aside from the presence of a bottom, the role of air is crucial for the observed differences between the impact phenomena near a pit and a pore. As soon as the pit is sealed off by the spreading liquid, the air inside will oppose 
liquid entering and therefore promote spreading and splashing. In order for liquid to enter the pit, air must be displaced by the ensuing pressure build-up inside the pit. Air might then leave either as a distinct air bubble or along with the liquid that jets out of the cavity. A common feature of impact near a pit and a pore is the splash that is observed. However, for the pore, the splash is only visible in a narrow $\Delta^{*}$ range, whereas for the pit, the full investigated $\Delta^{*}$ range is covered. We believe that this difference will at least be observed as long as the air inside the pit can be assumed to be incompressible, which we estimated to be valid for pit depths smaller than $85 \mathrm{~mm}$.

This chapter is entirely focused on the inertial regime, with We $\approx 370$ (and $\operatorname{Re} \approx 9 \times 10^{3}$ ) in almost all cases. We believe that the phenomena discussed in this chapter can be observed for the whole inertial regime (as long as no corona splashing occurs). Furthermore, the splash Weber number can be estimated as 30, also indicating that the phenomenon can still appear when surface tension is changed, for example. The jet, however, we expect to be mainly affected by a change in the impact Weber number, as the jet velocity reduces towards the sides of the $\Delta^{*}$ range shown in figure6.7. Hence, we expect that a reduced We number will give a smaller $\Delta^{*}$ range for which jets are observed. 


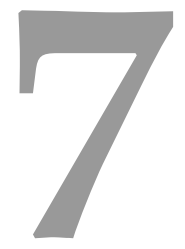

\section{Summary and outlook}

When a raindrop hits a sand bed, it leaves behind a small crater with a mixture of liquid and grains located (mainly) at the center. By studying this event in the lab with help of high speed laser profilometry, frequently accompanied with plain high speed imaging, we could elucidate various physics aspects of this ubiquitous phenomenon. We investigated the early stage of rain impacting on sand, i.e., a single drop impact on a still dry bed of grains.

As soon as a drop impacts on a granular bed, both the drop and the target start to deform, and for wettable grains mixing occurs. This is an in principle entangled state, where the behavior of the drop affects the crater formation, and vice versa, the behavior of the bed influences the drop response upon impact. This interplay will highly depend on the stiffness of both intruder and target, determined by e.g., the packing fraction of the bed. Throughout this thesis we showed that the drop deformation and the sand bed deformation could be studied relatively independently, by taking one measure into account that indicates the amount of entanglement: the maximum crater depth $Z_{c}^{*}$. As the droplet is decelerated slower for a deforming substrate than for a solid one, with $a=U_{0}^{2} /\left(D_{0}+2 Z_{c}^{*}\right)$, with $U_{0}$ the impact velocity, $D_{0}$ the drop size and $Z_{c}^{*}$ the maximum crater depth, we can use this deceleration to obtain an estimate of the stopping force of the drop. This stopping force serves subsequently as an input to estimate how much energy goes into either droplet deformation or sand deformation. Hence, we narrowed the entanglement down to a still experimentally obtained quantity, the maximum depth of 
the crater $Z_{c}^{*}$.

In chapter 2, we discussed the above described method in detail, showing that this partitioning indicates that the one that is most deformable (intruder or target) will absorb most of the impact energy. Furthermore, we showed that the maximum diameter of the spreading droplets follows a modified Weber number $\mathrm{We}^{\dagger / 4}$, a finding that confirmed the approach of using the deceleration of the drop as a measure, as in this line of reasoning it was proposed by Clanet et al. [39].

In this chapter we used hydrophilic glass beads, and hence, water and grains will mix. This may either be seen as liquid that penetrates between the grains or grains that get imbibed in the liquid phase. The mixing results into three types of muddy crater centers, that are denoted as a doughnut, a truffle and a pancake depending on their shape. We found that the dominating driving pressure of liquid into the bed is the inertial pressure of the impacting drop and used this pressure as an input into Darcy's law, which was used to describe the penetration of liquid into the pores between the sand grains. From this analysis we estimated the mixing time scale. By taking the ratio of the mixing time to the inertial time scale, we found that the transition from the compact truffle and doughnut shapes to the flat pancakes occurs when this ratio is equal to 1 . In this way, we showed that truffles and doughnuts occur when mixing is relatively slow and surface tension can still contract the liquid-grain residues into a compact shape, but when mixing becomes fast, the liquid is already for the largest part inside the sand bed before surface tension is able to contract the droplet, which results into flat pancake-shaped residues.

In chapter 3 , we delved further into the mixing between liquid and grains and studied how it affects the maximum droplet spreading and splashing. We changed a number of parameters that modify the capillary pull that the liquid experiences from the substrate, e.g., the wettability of the grains between very hydrophilic to hydrophobic, the grain size of the beads, and the surface tension of the liquid. Therefore, besides the inertial pressure, the capillary pressure becomes a key ingredient in explaining the mixing of liquid and grains, as it can prevent mixing for hydrophobic grains, but can contribute significantly to liquid penetration for a hydrophilic sand bed as well. When the liquid is pushed (or pulled) in-between the grains, the liquid flow is quickly slowed down by viscous dissipation. In this chapter we showed that the mixing of liquid and grains can be modeled as the development of a viscous boundary layer, analogous to (viscous) droplet spreading on a solid substrate and captured the effect of this bulk wettability into a modified Reynolds number $\mathrm{Re}^{\dagger}$. Similar to droplet spreading on a solid substrate, we could distinguish two spreading regimes: the first is a capillary regime with slow pene- 
tration of the liquid and the droplet spreading scaling with a power-law of $\mathrm{We}^{\dagger^{1 / 4}}$, and the second a viscous regime, where mixing stops the droplet spreading and the maximum droplet diameter collapses with $\mathrm{Re}^{\dagger 1 / 5}$. With the same modified dimensionless numbers, we showed that liquid-grain mixing suppresses liquid splashing from the lamella edge by means of a non-dimensional splash parameter $K_{d}^{\dagger}=\mathrm{We}^{\dagger 1 / 2} \mathrm{Re}^{\dagger^{1 / 4}}$ that captured the onset from deposition to splashing.

In chapter4, we shifted towards the dry part of the crater. We found that the estimated portion of the impact energy that goes into sand deformation (chapter2) can be used to describe and collapse the relevant crater length scales. However, regarding the final crater diameter we observed that for loose beds and high impact velocities the data deviates. We therefore investigated the second part of the formation of the crater, by comparing the crater at the moment it reaches its maximum depth and the final crater shape, to see if we could find an answer on why deviations in the final crater diameter occur. Interestingly, we saw that for the high energy impacts on loose beds, the transient crater has the steepest slopes, where final crater angles are all close to the angle of repose. Using a measure of the crater volume difference, we postulated that in this regime avalanches of grains become significant in modifying the final crater diameter. A last conclusion in this chapter is that the crater depth and diameter respond differently to the impact, such that the ratio of these length-scales displayed a distinct packing fraction dependence and a small variation with impact energy.

The investigation on crater formation continued in chapter 5 Here we compared the impact of a deformable water droplet on hydrophobic sand with that of a solid ball of similar size and density as the drop onto the same substrate. We found that the solid intruder impacts resulted in deeper but narrower craters than the droplet induced craters. We disproved the intuitive idea that the crater diameter for droplets can be related to the maximum droplet spreading and hence, for that reason may be broader than the ones caused by a solid intruder. In contrast, we found that, where mixing clearly decreases the liquid spreading (chapter3), the crater diameter does not get measurably smaller. We believe that mixing only limits crater formation when the liquid directly penetrates into the bed or when the granular bed is very densely packed and only the top layer of grains is affected by droplet spreading, i.e., when hardly any momentum transfer to the sand occurs. Despite the observed difference in the crater dimensions, we found for dense beds of $\phi_{0}>0.58$ that the excavated crater volume collapses for solid intruder and water droplet, when we take into account that for the droplet intruder part of the initial energy is consumed by drop deformation. This suggests that for dense beds, the energy dissipation in the sand is the same for a droplet and a solid intruder and is 
independent of the details of the crater formation process. That is to say, it only matters that a certain amount of grains is moved in the crater formation process, and not how.

Finally, in chapter 6, we moved away from granular matter as a target and looked into drop impact onto smooth surfaces containing a single large-scale roughness, namely a hole with a diameter that was similar but smaller than the size of the impacting droplet. Two types of holes were investigated, namely smooth surfaces containing a closed-end pit and those with an open-ended pore that went all the way through the substrate. For the pits, we found that by decreasing the distance of impact to the outer pit edge, the observed phenomenon changes from a splash only, via a splash together with a jet, to a splash and the entrapment of an air bubble. For the pores, however, we only observe splashing. We showed that the splash is formed at the outer pit or pore edge, and hence, is plausible to occur for both pit and pore. For the other two pit phenomena, the air bubble and the jet, we need an enclosed air pocket or a flow-deflecting pit bottom respectively, and are thus neither expected nor observed for open-ended pores. For the pore splash, we surprisingly detected two distinct splash regimes, dependent on the impact location, but the pits always produced splashes. This indicates that the air present inside the pit is crucial, not only for the formation of jets and air bubbles, but also for the occurrence of the splash. This idea is further confirmed by the difference in splash velocity that was reached for pit and pore splashes.

With this thesis, we have managed to answer various open questions in the field of drop impact on sand. We could explain the effect of mixing on the droplet spreading, splashing and final residue shape. We found that mixing does not affect the dry part of the crater, but that the ability of the drop to deform broadens the crater and makes it shallower than for a solid intruder. We saw that avalanches are a relevant restoring force of the granular media, and can contribute significantly to the final shape of the crater. And we found an experimental partition of the initial kinetic energy into sand and drop deformation energy. Despite all that, various open questions still remain.

In chapter 2, but also in other work [14, 17, 85], various liquid-grain morphologies were reported: a doughnut/ring, a truffle/bump, a sink, a pancake and a pie shape, which were (in part) nicely exposed in a Gallery of Fluid Motion movie in 2010 [142]. In this thesis, we explained the transition from the curved doughnut and truffle shapes towards the pancake shapes. The sinks, which occur for small cohesive grains $(<50 \mu \mathrm{m})$, were captured in [85]. However, the differentiation between a pancake and a pie (i.e., a pancake with a upwardly curved center part) is still not understood. 
In chapters 2 and 3 , we found a theoretical base to explain the effects of a granular substrate on the spreading behavior of the droplet after it impacts. We do not have a theoretical prediction yet for the inverse; i.e., can we also model the influence the deforming droplet shape has on the formation of the crater? In chapter 5. we took some first steps by making a comparison between droplet and solid intruders and found that the drops make shallower, but broader craters. In the literature 176, 78, 143], the effect of the (solid) intruder shape on the granular bed is investigated. Stevens Bester \& Behringer [78] proposed a collisional threedimensional model that captures the influence of the intruder on the penetration depth and, despite the fact that they study much higher impact energies of several Joules rather than the microJoules of our experiment, an interesting extension would be to incorporate a shape change in time, to see if we can explain the maximum depth reached for deformable droplets. Another approach might be to estimate the various drag force components for this low-energy case. Experimentally, it will be of help to modify the intruder stiffness in steps, e.g., by changing the liquid viscosity or by taking deformable balls, such as hydrogels. Besides experimental work, numerical simulations of droplet impact on sand could greatly contribute to our understanding of how momentum is transferred from droplet to sand. Where numerical work has elucidated our knowledge of the impact of solid objects on sand by studying the problem at the single-grain scale [144, 145], similar simulations using a continuously deforming intruder would be welcome.

Additionally, for the typical crater dimensions, various power-law scalings have been reported, see chapter 4. We proposed a plausible reason why there is a variety in scaling laws exponents reported in the literature, but could not make a prediction for the expected scaling exponent. More research is needed to capture the dimensionless quantities that set the problem and solve the quest on the proper power-law scaling for droplets on sand.

One of the findings in this thesis is that avalanches can affect the final crater shape. As a matter of fact, this is one example of what can complicate the just mentioned search to obtain a power-law scaling. It would be interesting to see if the volume change that can be contributed to avalanches can be measured and also predicted for this axisymmetric situation.

For droplet impact, we managed to capture the influence of the packing fraction of the bed, into a "deformability factor", i.e., the experimentally measured prefactor that we proposed to partition the initial kinetic energy of the drop into sand deformation and droplet deformation energy. How the packing fraction exactly affects the stiffness of the sand bed is not yet understood. Furthermore, the packing fraction plays a role in various properties of a granular bed, e.g., the amount 
of contacts that are present, the stiffness of the bed, the ease with which its grains are moved (thinking of, e.g., dilatancy [66]), but the role of packing fraction is not yet well captured in a model. In addition to the geometrical constraint imposed for high packing fraction (dilatancy), the internal contact network of a packing influences its force response as well. For frictional particles, this contact network depends on the preparation method and history and is not simply obtainable in dry three-dimensional experiments $[60,146]$. Despite the enormous challenge it poses, it is essential to grasp and hopefully predict the role of the packing of the bed better, as fine desert sand, river sand or coarse beach sand will all respond differently upon impact.

Two particularly relevant topics were left undiscussed in this thesis, namely the possible effect of the intruder to grain size ratio and the splash of grains. The first is a measure for the amount of contacts between intruder and grains during impact, and can therefore be of relevance in understanding a major source of dissipation of the impact energy. The granular splash is expected to affect erosion, as relevant nutrients could splash out, and subsequently by sheet erosion or wind flow get transported away from the land [1, 2]. Others [3, 15, 20, 112, 147] have investigated it, where Long et al. [20] indicated that for their impact parameters, a maximum of $4 \%$ of the initial droplet momentum goes into splashing. A full study is however still lacking.

Lastly, we found for droplet impact near closed-bottom pits and open-ended pores that the splash velocities of pit and pore differ significantly and we hypothesized that this is due to the presence of air for the pits that causes this increased speeds. Experiments with altered ambient air pressure could elucidate this topic, maybe in combination with an attempt on modeling the splash velocity with the model of Riboux and Gordillo [47, 148, 149], but then taking into account that the air is dragging the liquid along.

In conclusion, in this thesis we have striven to make progress in understanding droplet impact on sand by disentangling the three basic processes that this phenomenon consists of, namely droplet deformation, sand deformation, and mixing. In this way we managed to shed light on the physics underlying this beautiful but complicated process, in a similar way as how one may understand the famous illusion of figure 1.3 in the introduction, where both the young and elder lady are seen to coexist, but the individual traits may be observed as well. 


\section{Bibliography}

[1] B. W. Carey, B. Stone, P. L. Norman, and P. Shilton, "Soil Conservation Guidelines for Queensland”, Technical Report, State of Queensland, Department of Science, Information Technology and Innovation, Brisbane (2015).

[2] N. Evelpidou and A. Vassilopoulos, "Types of water erosion", in Soil protection in sloping mediterranean agri-environments: Lectures and exercises, edited by N. Evelpidou and T. Figueiredo, 53-66, Instituto Politécnico de Bragança, BragançaPortugal (2009).

[3] M. M. Al-Durrah and J. M. Bradford, "The mechanism of raindrop splash on soil surfaces”, Soil Sci. Soc. Am. J. 46, 1086-1090 (1982).

[4] P. I. A. Kinnell, “The mechanics of raindrop induced flow transport”, Soil Res. 28, 497-516 (1990).

[5] P. I. A. Kinnell, "Raindrop-impact-induced erosion processes and prediction: a review”, Hydrol. Process. 19, 2815-2844 (2005).

[6] C. A. E. Hamlett, S. Atherton, N. J. Shirtcliffe, G. McHale, S. Ahn, S. H. Doerr, R. Bryant, and M. I. Newton, "Transitions of water-drop impact behaviour on hydrophobic and hydrophilic particles”, Eur. J. Soil Sci. 64, 324-333 (2013).

[7] H. Ghadiri, "Crater formation in soils by raindrop impact", Earth Surf. Process. Landforms 29, 77-89 (2004).

[8] D. J. Furbish, K. K. Hamner, M. Schmeeckle, M. N. Borosund, and S. M. Mudd, "Rain splash of dry sand revealed by high-speed imaging and sticky paper splash targets", J. Geophys. Res. 112, F01001 (2007).

[9] S. Assouline, "Drop size distributions and kinetic energy rates in variable intensity rainfall”, Water Resour. Res. 45, W11501 (2009).

[10] K. P. Hapgood and B. Khanmohammadi, "Granulation of hydrophobic powders", Powder Technology 189, 253 - 262 (2009), special Issue: 3rd International Workshop on Granulation: Granulation across the Length Scales. 
[11] H. N. Emady, D. Kayrak-Talay, W. C. Schwerin, and J. D. Litster, “Granule formation mechanisms and morphology from single drop impact on powder beds", Powder Technol. 212, 69-79 (2011).

[12] C. M. Cejas, Y. Wei, R. Barrois, C. Fretigny, D. J. Durian, and R. Dreyfus, "Kinetics of gravity-driven water channels under steady rainfall”, Phys. Rev. E 90, 042205 (2014).

[13] Y. Wei, C. M. Cejas, R. Barrois, R. Dreyfus, and D. J. Durian, "Morphology of rain water channeling in systematically varied model sandy soils”, Phys. Rev. Applied 2, 044004 (2014).

[14] H. Katsuragi, "Morphology scaling of drop impact onto a granular layer", Phys. Rev. Lett. 104, 218001 (2010).

[15] J. Marston, S. Thoroddsen, W. Ng, and R. Tan, "Experimental study of liquid drop impact onto a powder surface”, Powder Technol. 203, 223-236 (2010).

[16] H. Katsuragi, "Length and time scales of a liquid drop impact and penetration into a granular layer", J. Fluid Mech. 675, 552-73 (2011).

[17] G. Delon, D. Terwagne, S. Dorbolo, N. Vandewalle, and H. Caps, "Impact of liquid droplets on granular media", Phys. Rev. E 84, 046320 (2011).

[18] E. Nefzaoui and O. Skurtys, "Impact of a liquid drop on a granular medium: Inertia, viscosity and surface tension effects on the drop deformation”, Exp. Therm. Fluid Sci. 41, 43 - 50 (2012).

[19] J. O. Marston, Y. Zhu, I. U. Vakarelski, and S. T. Thoroddsen, "Deformed liquid marbles: Freezing drop oscillations with powders”, Powder Technol. 228, 424-428 (2012).

[20] E. J. Long, G. K. Hargrave, J. R. Cooper, B. G. B. Kitchener, A. J. Parsons, C. J. M. Hewett, and J. Wainwright, "Experimental investigation into the impact of a liquid droplet onto a granular bed using three-dimensional, time-resolved, particle tracking”, Phys. Rev. E 89, 032201 (2014).

[21] R. Zhao, Q. Zhang, H. Tjugito, and X. Cheng, "Granular impact cratering by liquid drops: Understanding raindrop imprints through an analogy to asteroid strikes", Proc. Natl. Acad. Sci. 112, 342-347 (2015).

[22] Image of drip irrigation taken from www.plantnativ.com/services/drip-irrigation/, accessed on the 30th of March 2017.

[23] Image of sprinkler irrigation taken from www.dynamicautomation.com/wpcontent/uploads/2013/ 10/irrigation-sm.jpg, accessed on the 16th of April 2017.

[24] Erosion image taken from soilerosion.net/doc/water_erosion.html, accessed on the 30th of March 2017. 
[25] Soil erosion on website government Queensland, Australia; www.qld.gov.au/environment/land/soil/erosion/types/, accessed on the 24th of March 2017.

[26] W. E. Hill, "My wife and my mother-in-law”, Puck 16, 11 (1915).

[27] Image of young vs old lady taken from poligo.com/en/articles/vocabulary/howuse-see-look-and-watch-english, accessed on the 25th of March 2017.

[28] A. M. Worthington, "On the forms assumed by drops of liquids falling vertically on a horizontal plate”, P. R. Soc. London 25, 261- 72 (1876).

[29] M. Rein, "Phenomena of liquid drop impact on solid and liquid surfaces", Fluid Dyn. Res. 12, 61 - 93 (1993).

[30] A. L. Yarin, “Drop impact dynamics: Splashing, spreading, receding, bouncing...”, Annu. Rev. Fluid Mech. 38, 159-92 (2006).

[31] C. Josserand and S. T. Thoroddsen, "Drop Impact on a Solid Surface”, Annu. Rev. Fluid Mech. 48, 365-391 (2016).

[32] S. Chandra and C. T. Avedisian, "On the Collision of a Droplet with a Solid Surface", P. Roy. Soc. Lond. A Mat. 432, 13-41 (1991).

[33] M. Pasandideh-Fard, Y. M. Qiao, S. Chandra, and J. Mostaghimi, "Capillary effects during droplet impact on a solid surface”, Phys. Fluids 8, 650 (1996).

[34] T. Mao, D. C. S. Kuhn, and H. Tran, "Spread and rebound of liquid droplets upon impact on flat surfaces”, AIChE J. 43, 2169-2179 (1997).

[35] I. V. Roisman, "Inertia dominated drop collisions. II. An analytical solution of the Navier-Stokes equations for a spreading viscous film”, Phys. Fluids 21, 052104 (2009).

[36] J. Eggers, M. A. Fontelos, C. Josserand, and S. Zaleski, "Drop dynamics after impact on a solid wall: Theory and simulations”, Phys. Fluids 22, 062101 (2010).

[37] J. B. Lee, N. Laan, K. G. de Bruin, G. Skantzaris, N. Shahidzadeh, D. Derome, J. Carmeliet, and D. Bonn, "Universal rescaling of drop impact on smooth and rough surfaces”, J. Fluid Mech. 786, R4 (2016).

[38] S. Wildeman, C. W. Visser, C. Sun, and D. Lohse, "On the spreading of impacting drops", J. Fluid Mech. 805, 636-655 (2016).

[39] C. Clanet, C. Béguin, D. Richard, and D. Quéré, "Maximal deformation of an impacting drop”, J. Fluid Mech. 517, 199-208 (2004). 
[40] J. Madejski, “Solidification of droplets on a cold surface”, Int. J. Heat Mass Trans. 19, 1009 - 1013 (1976).

[41] G. Trapaga and J. Szekely, "Mathematical modeling of the isothermal impingement of liquid droplets in spraying processes”, Metall. Trans. B 22, 901-914 (1991).

[42] N. Laan, K. G. de Bruin, D. Bartolo, C. Josserand, and D. Bonn, "Maximum diameter of impacting liquid droplets”, Phys. Rev. Applied 2, 044018 (2014).

[43] D. Bartolo, C. Josserand, and D. Bonn, "Retraction dynamics of aqueous drops upon impact on non-wetting surfaces”, J. Fluid Mech. 545, 329-338 (2005).

[44] P. Attané, F. Girard, and V. Morin, "An energy balance approach of the dynamics of drop impact on a solid surface”, Phys. Fluids 19, 012101 (2007).

[45] P. Tsai, M. H. W. Hendrix, R. R. M. Dijkstra, L. Shui, and D. Lohse, "Microscopic structure influencing macroscopic splash at high Weber number", Soft Matter 7, 1132511333 (2011).

[46] L. Xu, W. Zhang, and S. R. Nagel, "Drop splashing on a dry smooth surface”, Phys. Rev. Lett. 94, 184505 (2005).

[47] G. Riboux and J. M. Gordillo, "Experiments of drops impacting a smooth solid surface: A model of the critical impact speed for drop splashing”, Phys. Rev. Lett. 113, 024507 (2014).

[48] Y. Liu, P. Tan, and L. Xu, "Kelvin-Helmholtz instability in an ultrathin air film causes drop splashing on smooth surfaces”, Proc. Natl. Acad. Sci. 112, 3280-3284 (2015).

[49] C. Mundo, M. Sommerfeld, and C. Tropea, "Droplet-wall collisions: Experimental studies of the deformation and breakup process”, Int. J. Multiphase Flow 21, 151173 (1995).

[50] A. N. Lembach, H. B. Tan, I. V. Roisman, T. Gambaryan-Roisman, Y. Zhang, C. Tropea, and A. L. Yarin, "Drop impact, spreading, splashing, and penetration into electrospun nanofiber mats”, Langmuir 26, 9516-9523 (2010).

[51] Sand dunes image taken from hvo.wr.usgs.gov/volcanowatch/archive/2005/images/ sand_dunes.jpg, accessed on the 25th of March 2017.

[52] Image of a ball pit taken from www.gezondheidenco.nl/274480/indoor-speeltuinis-vaak-onveilig/, accessed on the 25th of March 2017.

[53] Landslide image taken from www.bgs.ac.uk/landslides/holbeckhall.html, accessed on the 25th of March 2017. 
[54] Image of rotating drum experiment taken from website of Olivier Dauchot www.drecam.cea.fr/spec/Pres/Git/GM/gm.htm, accessed on the 27th of March 2017.

[55] Image of vulcano burst Calbuco taken from www.nu.nl/buitenland/4036107/vulkaancalbuco-in-chili-barst.html, accessed on the 25th of March 2017.

[56] H. M. Jaeger, S. R. Nagel, and R. P. Behringer, “Granular solids, liquids, and gases”, Rev. Mod. Phys. 68, 1259-73 (1996).

[57] L. P. Kadanoff, “Granular flows”, Rev. Mod. Phys. 71, 435-44 (1999).

[58] P. G. de Gennes, “Granular matter : a tentative view”, Rev. Mod. Phys. 71, 374-382 (1999).

[59] I. S. Aranson and L. S. Tsimring, "Patterns and collective behavior in granular media: Theoretical concepts”, Rev. Mod. Phys. 78, 641-692 (2006).

[60] B. Andreotti, Y. Forterre, and O. Pouliquen, Granular media: between fluid and solid, Cambridge University Press (2013).

[61] A. H. Clark, A. J. Petersen, L. Kondic, and R. P. Behringer, "Nonlinear Force Propagation During Granular Impact”, Phys. Rev. Lett. 114, 144502 (2015).

[62] D. Lohse, R. Rauhé, R. Bergmann, and D. van der Meer, "Creating a dry variety of quicksand", Nature 432, 689 (2004).

[63] Q. Zhang and K. Kamrin, "Microscopic description of the granular fluidity field in nonlocal flow modeling”, Phys. Rev. Lett. 118, 058001 (2017).

[64] S. Luding, "Towards dense, realistic granular media in 2d", Nonlinearity 22, R101 (2009).

[65] D. Vescovi and S. Luding, "Merging fluid and solid granular behavior", Soft Matter 12, 8616-8628 (2016).

[66] M. Schröter, S. Nägle, C. Radin, and H. L. Swinney, "Phase transition in a static granular system", Europhys. Lett. 78, 44004 (2007).

[67] P. Thompson and G. Grest, “Granular flow: Friction and the dilatancy transition.”, Phys. Rev. Lett. 67, 1751-1754 (1991).

[68] P. Umbanhowar and D. I. Goldman, "Granular impact and the critical packing state", Phys. Rev. E 82, 010301 (2010).

[69] K. A. Holsapple and R. M. Schmidt, "Point source solutions and coupling parameters in cratering mechanics”, J. Geophys. Res. 92, 6350-6376 (1987). 


\section{BIBLIOGRAPHY}

[70] H. J. Melosh, Impact cratering: A geologic process, volume 11, Oxford University Press, New York (1989).

[71] J. C. Ruiz-Suárez, "Penetration of projectiles into granular targets”, Rep. Prog. Phys. 76, 066601 (2013).

[72] H. Katsuragi, Physics of Soft Impact and Cratering, Springer Japan (2016).

[73] D. van der Meer, "Impact on Granular Beds", Annu. Rev. Fluid Mech. 49, 463-84 (2017).

[74] M. A. Ambroso, C. R. Santore, A. R. Abate, and D. J. Durian, "Penetration depth for shallow impact cratering", Phys. Rev. E 71, 051305 (2005).

[75] H. Katsuragi and D. J. Durian, "Unified force law for granular impact cratering”, Nat. Phys. 3, 420-423 (2007).

[76] A. H. Clark, A. J. Petersen, and R. P. Behringer, "Collisional model for granular impact dynamics", Phys. Rev. E 89, 012201 (2014).

[77] Y. Xu, J. T. Padding, and J. A. M. Kuipers, "Numerical investigation of the vertical plunging force of a spherical intruder into a prefluidized granular bed", Phys. Rev. E 90, 062203 (2014).

[78] C. Stevens Bester and R. P. Behringer, "Collisional model of energy dissipation in three-dimensional granular impact”, Phys. Rev. E 95, 032906 (2017).

[79] K. A. Holsapple, “The scaling of impact processes in planetary sciences”, Annu. Rev. Earth Planet. Sci. 21, 333-373 (1993).

[80] J. C. Amato and R. E. Williams, "Crater formation in the laboratory: An introductory experiment in error analysis”, Am. J. Phys. 66, 141-143 (1998).

[81] A. M. Walsh, K. E. Holloway, P. Habdas, and J. R. de Bruyn, "Morphology and Scaling of Impact Craters in Granular Media”, Phys. Rev. Lett. 91, 104301 (2003).

[82] J. S. Uehara, M. A. Ambroso, R. P. Ojha, and D. J. Durian, "Low-speed impact craters in loose granular media”, Phys. Rev. Lett. 90, 194301 (2003).

[83] S. J. de Vet and J. R. de Bruyn, "Shape of impact craters in granular media”, Phys. Rev. E 76, 041306 (2007).

[84] H. Darcy, Les fontaines publiques de la ville de Dijon: exposition et application..., Victor Dalmont (1856).

[85] H. N. Emady, D. Kayrak-Talay, and J. D. Litster, "A regime map for granule formation by drop impact on powder beds”, AIChE J. 59, 96-107 (2013). 
[86] H. N. Emady, D. Kayrak-Talay, and J. D. Litster, "Modeling the granule formation mechanism from single drop impact on a powder bed.", J. Colloid Interface Sci. 393, 369-76 (2013).

[87] D. Lohse, R. Bergmann, R. Mikkelsen, C. Zeilstra, D. van der Meer, M. Versluis, K. van der Weele, M. van der Hoef, and H. Kuipers, "Impact on soft sand: Void collapse and jet formation”, Phys. Rev. Lett. 93, 198003 (2004).

[88] N. Gravish, P. B. Umbanhowar, and D. I. Goldman, "Force and Flow Transition in Plowed Granular Media”, Phys. Rev. Lett. 105, 128301 (2010).

[89] E. Villermaux and B. Bossa, "Single-drop fragmentation determines size distribution of raindrops”, Nat. Phys. 5, 697-702 (2009).

[90] J.-F. Métayer, D. J. Suntrup III, C. Radin, H. L. Swinney, and M. Schröter, “Shearing of frictional sphere packings", Europhys. Lett. 93, 64003 (2011).

[91] D. Soto, A. B. De Larivière, X. Boutillon, C. Clanet, and D. Quéré, “The force of impacting rain.", Soft matter 10, 4929-34 (2014).

[92] T. Bennett and D. Poulikakos, "Splat-quench solidification: estimating the maximum spreading of a droplet impacting a solid surface", J Mater Sci 28, 963-970 (1993).

[93] A. M. Worthington, Study of splashes, chapter 7, Longmans, Green and Co., London (1908).

[94] S. C. Zhao, R. de Jong, and D. van der Meer, "Raindrop impact on sand: a dynamic explanation of crater morphologies", Soft Matter 11, 6562-6568 (2015).

[95] R. de Jong, S.-C. Zhao, and D. van der Meer, "Crater formation during raindrop impact on sand”, Phys. Rev. E 95, 042901 (2017).

[96] Y. S. Joung and C. R. Buie, “Aerosol generation by raindrop impact on soil”, Nat. Commun. 6, 6083 (2015).

[97] M. Denesuk, G. L. Smith, B. J. J. Zelinski, N. J. Kreidl, and D. R. Uhlmann, “Capillary Penetration of Liquid Droplets into Porous Materials”, J. Colloid Interface Sci. 158, 114-120 (1993).

[98] P. Carman, Flow of gases through porous media, Butterworths, London (1956).

[99] D. Richard, C. Clanet, and D. Quéré, "Surface phenomena: Contact time of a bouncing drop", Nature 417, 811 (2002).

[100] K. Okumura, F. Chevy, D. Richard, D. Quéré, and C. Clanet, "Water spring: A model for bouncing drops", Europhys. Lett. 62, 237 (2003). 


\section{BIBLIOGRAPHY}

[101] K. Range and F. Feuillebois, "Influence of Surface Roughness on Liquid Drop Impact”, J. Colloid Interface Sci. 203, 16-30 (1998).

[102] R. Rioboo, C. Tropea, and M. Marengo, "Outcomes from a drop impact on solid surfaces", At. Spray 11, 155-65 (2001).

[103] F. Brochard and P. G. De Gennes, "Shear-dependent slippage at a polymer/solid interface”, Langmuir 8, 3033-3037 (1992).

[104] M. Mani, S. Mandre, and M. P. Brenner, "Events before droplet splashing on a solid surface”, J. Fluid Mech. 647, 163-185 (2010).

[105] M. M. Driscoll and S. R. Nagel, "Ultrafast interference imaging of air in splashing dynamics”, Phys. Rev. Lett. 107, 154502 (2011).

[106] W. Bouwhuis, R. C. A. van der Veen, T. Tran, D. L. Keij, K. G. Winkels, I. R. Peters, D. van der Meer, C. Sun, J. H. Snoeijer, and D. Lohse, "Maximal air bubble entrainment at liquid-drop impact”, Phys. Rev. Lett. 109, 264501 (2012).

[107] J. de Ruiter, J. M. Oh, D. van den Ende, and F. Mugele, "Dynamics of collapse of air films in drop impact.”, Phys. Rev. Lett. 108, 074505 (2012).

[108] C. J. Howland, A. Antkowiak, J. R. Castrejón-Pita, S. D. Howison, J. M. Oliver, R. W. Style, and A. A. Castrejón-Pita, “It's Harder to Splash on Soft Solids”, Phys. Rev. Lett. 117, 184502 (2016).

[109] Q. Zhang, M. Gao, R. Zhao, and X. Cheng, "Scaling of liquid-drop impact craters in wet granular media”, Phys. Rev. E 92, 042205 (2015).

[110] S. C. Zhao, R. de Jong, and D. van der Meer, "Liquid-grain mixing suppresses droplet spreading and splashing during impact”, Phys. Rev. Lett. 118, 054502 (2017).

[111] R. J. Pike, "Formation of complex impact craters: Evidence from Mars and other planets", Icarus 43, 1-19 (1980).

[112] J. O. Marston, E. Q. Li, and S. T. Thoroddsen, "Evolution of fluid-like granular ejecta generated by sphere impact”, J. Fluid Mech. 704, 5-36 (2012).

[113] F. Pacheco-Vázquez and J. C. Ruiz-Suárez, "Impact craters in granular media: Grains against grains”, Phys. Rev. Lett. 107, 218001 (2011).

[114] J. F. Boudet, Y. Amarouchene, and H. Kellay, "Dynamics of Impact Cratering in Shallow Sand Layers”, Phys. Rev. Lett. 96, 158001 (2006).

[115] F. Bonnet, T. Richard, and P. Philippe, "Sensitivity to solid volume fraction of gravitational instability in a granular medium”, Granul. Matter 12, 317-325 (2010). 
[116] L. Rondon, O. Pouliquen, and P. Aussillous, "Granular collapse in a fluid: Role of the initial volume fraction”, Phys. Fluids 23, 073301 (2011).

[117] N. Gravish, P. B. Umbanhowar, and D. I. Goldman, "Force and flow at the onset of drag in plowed granular media”, Phys. Rev. E 89, 042202 (2014).

[118] T. G. Murthy, E. Gnanamanickam, and S. Chandrasekar, "Deformation field in indentation of a granular ensemble”, Phys. Rev. E 85, 061306 (2012).

[119] K. N. Nordstrom, E. Lim, M. Harrington, and W. Losert, “Granular Dynamics During Impact”, Phys. Rev. Lett. 112, 228002 (2014).

[120] S. J. de Vet and J. R. de Bruyn, "The collapse of a cylindrical cavity in a granular medium”, Granul. Matter 14, 661-670 (2012).

[121] T. Boutreux and P.-G. de Gennes, "Étalement d'une marche de sable: Le problème du sinaï”, C. R. Acad. Sci. Paris 325, 85-89 (1997).

[122] A. Aradian, É. Raphaël, and P.-G. de Gennes, "Surface flows of granular materials : a short introduction to some recent models”, C. R. Phys. 3 3, 187-196 (2002).

[123] R. M. Schmidt and K. R. Housen, "Some recent advances in the scaling of impact and explosion cratering”, Int. J. Impact Eng. 5, 543-560 (1987).

[124] R. Bartali, G. M. Rodríguez-Lin̂án, Y. Nahmad-Molinari, D. Sarocchi, and J. C. RuizSuárez, "Role of the granular nature of meteoritic projectiles in impact crater morphogenesis", arXiv:1302.0259 (2013).

[125] W. Bouwhuis, X. Huang, C. U. Chan, P. E. Frommhold, C.-D. Ohl, D. Lohse, J. H. Snoeijer, and D. van der Meer, "Impact of a high-speed train of microdrops on a liquid pool”, J. Fluid Mech. 792, 850-868 (2016).

[126] J. Aguilar and D. I. Goldman, "Robophysical study of jumping dynamics on granular media”, Nat Phys 12, 278-283 (2016).

[127] O. Reynolds, "Lvii. on the dilatancy of media composed of rigid particles in contact. with experimental illustrations”, Lond. Edinb. Dubl. Phil. Mag. 20, 469-481 (1885).

[128] P. Tsai, S. Pacheco, C. Pirat, L. Lefferts, and D. Lohse, "Drop impact upon micro- and nanostructured superhydrophobic surfaces”, Langmuir 25, 12293-12298 (2009).

[129] A. Delbos, E. Lorenceau, and O. Pitois, "Forced impregnation of a capillary tube with drop impact”, J Colloid Interf. Sci. 341, 171-177 (2010).

[130] H. Ding and T. G. Theofanous, "The inertial regime of drop impact on an anisotropic porous substrate”, J. Fluid Mech. 691, 546-567 (2012). 


\section{BIBLIOGRAPHY}

[131] É. Lorenceau and D. Quéré, “Drops impacting a sieve”, J Colloid Interf. Sci. 263, 244 -49 (2003).

[132] I. V. Roisman, C. M. Weickgenannt, A. N. Lembach, and C. Tropea, "Drop impact close to a pore: experimental and numerical investigations.", in Proceedings of the 23rd Annual Conference on Liquid Atomization and Spray Systems, ILASS-Europe (2010).

[133] C. Josserand, L. Lemoyne, R. Troeger, and S. Zaleski, "Droplet impact on a dry surface: triggering the splash with a small obstacle”, J. Fluid Mech. 524, 47-56 (2005).

[134] L. Xu, L. Barcos, and S. Nagel, "Splashing of liquids: Interplay of surface roughness with surrounding gas”, Phys. Rev. E 76, 066311 (2007).

[135] H. J. Subramani, T. Al-Housseiny, A. U. Chen, M. Li, and O. A. Basaran, "Dynamics of drop impact on a rectangular slot”, Ind. Eng. Chem. Res. 46, 6105-12 (2007).

[136] C. Josserand and S. Zaleski, "Droplet splashing on a thin liquid film”, Phys. Fluids 15, 1650-1657 (2003).

[137] S. T. Thoroddsen, K. Takehara, and T. G. Etoh, “Micro-splashing by drop impacts”, J. Fluid Mech. 706, 560-570 (2012).

[138] S. T. Thoroddsen, “The ejecta sheet generated by the impact of a drop", J. Fluid Mech. 451, 373-381 (2002).

[139] R. Rioboo, M. Marengo, and C. Tropea, "Time evolution of liquid drop impact onto solid, dry surfaces", Exp. Fluids 33, 112-124 (2002).

[140] A. Mongruel, V. Daru, F. Feuillebois, and S. Tabakova, "Early post-impact time dynamics of viscous drops onto a solid dry surface”, Phys. Fluids 21, 032101 (2009).

[141] H. Wagner, “Über stoß- und gleitvorgänge an der oberfläche von flüssigkeiten”, Z. Angew. Math. Mech. 12, 193-215 (1932).

[142] G. Delon, D. Terwagne, N. Adami, A. Bronfort, N. Vandewalle, S. Dorbolo, and H. Caps, "Freezing splashes", arXiv:1010.3139 (2010).

[143] T. A. Brzinski III, P. Mayor, and D. J. Durian, “Depth-dependent resistance of granular media to vertical penetration”, Phys. Rev. Lett. 111, 168002 (2013).

[144] L. Kondic, X. Fang, W. Losert, C. S. O’Hern, and R. P. Behringer, "Microstructure evolution during impact on granular matter”, Phys. Rev. E 85, 011305 (2012).

[145] A. H. Clark, A. J. Petersen, L. Kondic, C. O’Hern, and R. P. Behringer, “Granular impact: A grain-scale approach", arXiv:1611.00737 (2016). 
[146] M. van Hecke, "Jamming of soft particles: geometry, mechanics, scaling and isostaticity”, J. Phys. - Condens. Mat. 22, 033101 (2010).

[147] G. A. Caballero-Robledo, K. P. D. Kelly, T. A. M. Homan, J. H. Weijs, D. van der Meer, and D. Lohse, "Suction of splash after impact on dry quick sand", Granul. Matter 14, 179-184 (2012).

[148] G. Riboux and J. M. Gordillo, "Erratum: Experiments of drops impacting a smooth solid surface: A model of the critical impact speed for drop splashing [phys. rev. lett. 113, 024507 (2014)]”, Phys. Rev. Lett. 113, 189901 (2014).

[149] G. Riboux and J. M. Gordillo, "The diameters and velocities of the droplets ejected after splashing”, J. Fluid Mech. 772, 630-648 (2015). 



\section{Samenvatting}

Regendruppels die inslaan op zand veroorzaken kleine kraters met in het midden wat modder, een fenomeen dat we in Nederland met enige regelmaat kunnen waarnemen. Regen kan een grote impact op de zandgrond hebben, want door de hoge inslagsnelheid van de druppels kan het aanzetten tot erosie en daarmee nadelig zijn voor de groei van gewassen. Als regen echter uitblijft, kiest men vaak voor besproeiing om de planten van afdoende watertoevoer te verzekeren. Het voordeel van irrigatie is dat het gecontroleerd kan worden toegepast. Dit is vooral gunstig als het proces ook begrepen wordt en daarmee effectief toegepast kan worden. Kennis van de precieze interactie tussen vloeistof en korrels kan ook van belang zijn in industriële processen zoals in het farmaceutische productieproces van pillen. Van een andere orde zijn de kraters die gevormd zijn door de inslag van asteroïdes op o.a. de maan. Tijdens de inslag ontstaan echter hoge temperaturen en in de meeste gevallen wordt de asteroïde vloeibaar en eventueel zelfs gasvormig 170]. De vorming van kraters door druppelinslag kan dus als inspiratie dienen in de begripsvorming van deze planetaire kraters, welke door een vervormbaar object worden gecreëerd.

In dit proefschrift hebben we de inslag van een enkele druppel op een droog zandbed onderzocht. Dit is een complex en subtiel proces, want zodra de druppel inslaat op het zand vervormt zowel de druppel als het substraat, en bovendien vermengen de korrels en de vloeistof zich in het centrum van de krater die dan wordt gevormd. Daarbij is het van belang om te ontrafelen wat de invloed is die de druppelvervorming op het kratervormingsproces heeft, en vice versa, wat voor weerslag het gedrag van het zandbed heeft op het uitspreiden van de druppel.

Een bevinding die in het het gehele proefschrift terugkomt, is dat de initiële energie van de druppel kan worden opgesplitst in een afschatting van het deel van de energie dat naar druppelvervorming gaat en het gedeelte dat in de vervorming van het zandbed gaat zitten, waardoor de beide processen relatief onafhankelijk van elkaar bestudeerd kunnen worden.

De vervorming van de druppel en het mengen van vloeistof en zand is de focus van het eerste deel van het proefschrift. In hoofdstuk 2 wordt aangetoond dat de 
uiteindelijke vorm van het water-zand mengsel in het centrum van de krater afhangt van hoe snel deze twee zich vermengen. Op het moment dat er weinig korrels in de druppel worden geabsorbeerd, zal de druppel nadat deze is uitgespreid zich onder de invloed van de oppervlaktespanning terugtrekken tot een compacte vorm zoals een donut of een truffel, om zo de hoge kromming aan de buitenrand te elimineren. Als de snelheid van de druppel voor de inslag zo hoog is dat de vloeistof snel tussen de korrels indringt, vinden we dat zodra het vermengde volume aan korrels en vloeistof een kritische waarde bereikt, de invloed van de kromming aan de rand voldoende wordt afgezwakt, zodat de druppel zich niet meer kan terugtrekken en het mengsel een platte pannenkoekachtige vorm zal aannemen. We tonen aan dat de overgang tussen deze twee typen plaatsvindt wanneer de verhouding tussen twee tijdschalen gelijk is aan een. Dit zijn de tijdsduur van de inslag, die een gevolg is van de massatraagheid van de druppel, en de tijdschaal waarop zandkorrels en water zich vermengen.

In hoofdstuk 3 kijken we vervolgens naar de invloed van de vermenging op de maximale druppeluitspreiding door allerlei eigenschappen van het zandbed te variëren. We laten zien dat het mengproces kan worden gemodelleerd naar analogie van de ontwikkeling van een viskeuze grenslaag aan de onderkant van een druppel die zich uitspreidt op een vast oppervlak. Door deze benadering tonen we aan dat voor uitspreiding van de druppel op zand eenzelfde overgang plaatsvindt als voor de maximale druppeldiameter op een vast oppervlak, namelijk van een regio die gedomineerd wordt door de oppervlaktespanning naar een gebied waar de dissipatie van de initiële energie in de grenslaag substantieel is. Dit laatste gebeurt als druppel en zand zich snel vermengen. Deze vermenging blijkt ook het wegspatten van kleine druppeltjes aan de rand van de uitgespreide druppel tegen te kunnen gaan.

Vervolgens verplaatsen we de aandacht naar de vervorming van het droge zand en bestuderen in hoofdstuk 4 de typische lengteschalen van de krater, namelijk de diameter en de diepte. We laten zien dat variaties veroorzaakt door de pakkingsdichtheid van het zandbed grotendeels verklaard kunnen worden met de eerder genoemde energieverdeling naar druppel- en zandbedvervorming. Voor hoge inslagsnelheid en los op elkaar gepakt zand wijkt de data voor de kraterdiameter echter af. We tonen aan dat dit veroorzaakt wordt door kleine lawines van korrels aan de kraterwand, die zorgen voor een grotere uiteindelijke kraterdiameter. Ondanks dat in de literatuur vaak wordt aangenomen dat de ratio tussen kraterdiepte en -diameter constant is voor druppelinslag op zand, blijkt uit onze experimenten dat deze afneemt bij een toename in de pakkingsdichtheid van het zandbed.

In hoofdstuk 5 onderzoeken we de invloed van de vervormbaarheid van het 
invallende object door de inslag van een vervormbare druppel op zand te vergelijken met die van een balletje met eenzelfde grootte en dichtheid als de druppel. De kraters gevormd door druppelinslag blijken breder en ondieper te zijn dan die door niet-vervormbare balletjes. Het lijkt erop dat dit simpelweg een gevolg is van de intrinsieke eigenschap van een vloeibaar object om te vervormen, namelijk dat het vervormt als het een kracht ervaart. De druppel vervormt als gevolg van de kracht die er tijdens de inslag door het zandbed op wordt uitgeoefend en oefent daarbij als reactie weer een kracht op het substraat uit die niet alleen naar beneden toe gericht is, maar ook zijwaarts. Ook al verschillen de kraterdimensies voor bal- en druppelinslag van elkaar, de kratervolumes blijken verrassend genoeg voor relatief vast op elkaar gepakte korrels overeen te komen.

In het laatste hoofdstuk van dit proefschrift wordt geen zandbed gebruikt, maar een vast oppervlak waarin een kleine holte met een diameter in de ordegrootte van een millimeter aanwezig is. We vergelijken de inslag vlakbij een halfgesloten uitsparing met die vlakbij een gaatje dat open is aan de onderkant. Het blijkt dat voor beide type holtes vloeistof wegspat op het moment dat de uitspreidende druppel de rand van de holte bereikt, maar dat voor het half-gesloten kuiltje de hoeveelheid vloeistof en de snelheid ervan een stuk groter is dan voor een inslag vlakbij een compleet open holte. De invloed van de lucht die in de uitsparing aanwezig is, is de meest waarschijnlijke verklaring hiervoor. Deze kan bij een van onderen open holte immers simpelweg wegvloeien, maar in het geval van een kuiltje zal het via de opening bovenaan moeten wegstromen en zal daardoor de vloeistofstroom beïnvloeden.

In dit proefschrift laten we zien dat het ontrafelen van de drie basisprocessen die ten grondslag liggen aan druppelinslag op zand cruciaal is om tot een beter begrip van dit complexe en veelzijdige fenomeen te komen. 



\section{Acknowledgments}

How a wonder that time just continues and again spring has arrived at its fullest; the trees popped out their leaves again, the nights are light as well as the days, the flowers are, or have been, blooming in their full ability. I am thankful that life contains this natural cyclic change. It makes us adjust and keeps bringing new moments of amazement. Quite recently I experienced the great change of new life, from embryo to baby to one that moves and laughs and mumbles. Something wonderful to be part of and of which I am highly grateful.

My own life began thanks to my parents in 1986. Paps and mams, thanks for all the effort and the love that surrounded me during growing up. Mams, for your support in finding my own path. Paps, for your capability to relate to the many questions I can ask and for the concept that only asking a question can be enough. Bro, thanks for your being, for the ways you are so different than I am, and nonetheless we seem to get somehow more and more similar interests. Thanks HEMBJ, for living closer, for the kids stuff, for the liveliness, the humor. Growing up was not always easy, lucky me, I found some good friends that accompanied me and let me feel that being alone does not mean to be lonely (please read the wonderful booklet of David Grossman, the hug for this concept), and I felt surrounded. Thanks Jil, Wessel, Claudia for your friendship through already all these years.

When starting studying, new people entered my life with their thoughts, laughs, jokes, questions and activism. I am grateful for the time at the student association VGST and for the friendships that came along. For Bram, Jan, Elline, Jos, Tony, Wessel, Noortje, Petra, Wilco, Hinke, Annelies, Eline, Jaapjan, Arina. Annelies, thanks for the cover tips. I enjoyed the years at the modern dance association Arabesque tremendously. Thanks to Soosan for the knowledge on initiating movements from various body parts, and for all the creative folks that took part in the lessons, some with which still close friendships remain: Merel, Daniela, Veronica, Thea, thanks for being you. Living for a short period in Brasil eating pão de queijo and beans for lunch (what else?), I want to thank the many people we met for the openness and for the option to write the word saudades to you, Lívia, Liége, Fernando and others. 
Jos, my love, o meu amor, what a joy you came into my life already more then ten years ago and that you still are. As the poem from Michiel van der Plas says: "gewoon alleen door te bestaan doe jij mijn jaren mooie kleren aan". Dearest, it is great to have you near and feel your support encircling me. I am also thankful for the whole Havinga family (and co) I got with you. Jidde, you came into our life only recently. It is such a joy to have you near and see you discovering the world at its fullest.

At some point it was time to decide on a master track, which was in my case more focused on figuring out the best group for doing my nine months master research. Jacco, thanks for the first contact in my master decision-making process and later on, for the chats on teaching, life, and introductions. Detlef, your enthusiasm for science is engaging and I cherish that you focus on a diverse PoF team. Thanks to you, and unlike my manner, I knew already a year in advance which master research topic I would have, which attracted me into a PhD. Devaraj, it was great to have you as a supervisor. You are kind, precise and you act with care. I highly appreciate your focus on being as clear in writing as one can be, concise whenever possible but never loosing context. I am happy that PoF has approachable staff members. Chao, thanks for letting me put your window full of post-its and especially, for your liveliness. Leen, thanks for your kind attitude and for showing that a curious approach to life never ends. Joanita, you are always willing to give a quick help out, bedankt. Thanks Gert-Wim and Martin for the effort on the setup together with the TCO guys. Bas, for your always kind help on sometimes trivial computer related questions and the chats on campers. Antoinette, although you are not part of the PoF staff, you are around regularly. Thanks for the morning chats and your advise on child-raising stuff.

As I highly appreciate the quality of amazement and curiousness, I am happy I was surrounded by so many folks displaying this feature (or question it) in the Physics of Fluids group. It is a proper scientific surrounding in that manner. It is impossible to list all that made PoF a nice atmosphere to work in. If you are or were in PoF, thanks for the moments at the coffee machine, a corridor, office ME250, a lunch table, during an afternoon walk or conference (and accompanying travels) to have a chat, a scientific discussion, moments of valuable support or an in-depth talk. Wilco, Roeland, Enrique and Rodrigo, thanks for sharing the teaching experience in the Bachelor course on fluid mechanics. Michiel, it was nice to set up and organize the DROP meetings together. Song-Chuan, or a.k.a. my post-doc, it was great to have you near for discussions of any kind, but especially for all clever thoughts you had when I needed some input on my work. I am still amazed on how wisely you can choose on the things you will do quick and dirty and which 
ones precise. Hanneke, we had a proper take off of an in-depth friendship in Denmark in 2013, before visiting the Krøgerup summer school. I am happy that we still share life regularly.

I had the honor to learn and develop myself by supervising some students while they were doing Experimental Techniques experiments or Bachelor research. Thanks Carlijn, Benjamin, Mike, Myrthe, Elise, Diana, Stefan, Maarten and Gijs. I am grateful for the effort of the last six to carry out and improve the solid ball experiments which made it (in part) into chapter5,

Last but not least, I am thankful for all of you that come and support me during my defense. I would like to end with a Portuguese sentence a friend once told me, summarizing these acknowledgments: "A vida é um vai e vem de gente que nos faz sentir saudades e ter certeza que todos os dias valem a pena", which roughly means: life is a coming and going of people which we miss and love, what ensures us that every day is worth living.

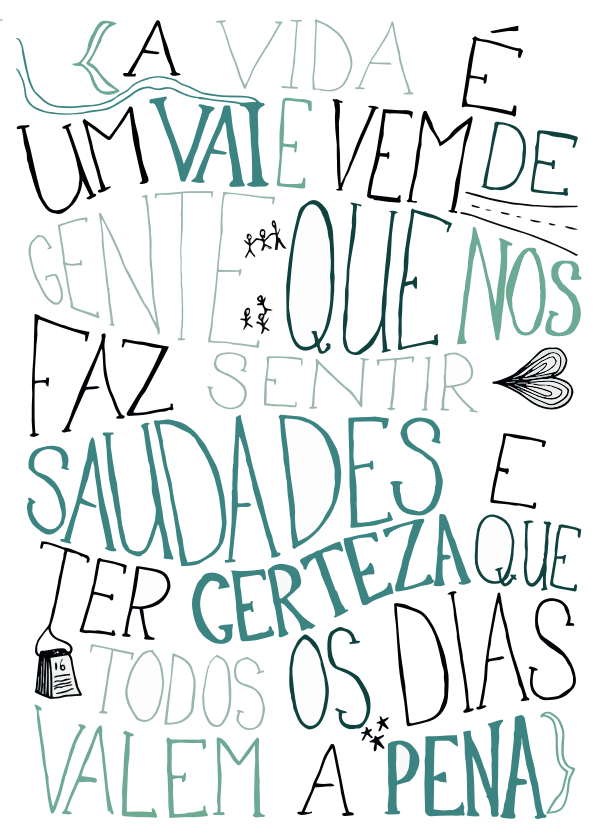



